\title{
Atlas of high-resolution emission and shell lines in Be stars. Line profiles and short-term variability ${ }^{\star}$
}

\author{
R.W. Hanuschik ${ }^{1,2}$, W. Hummel ${ }^{3}$, E. Sutorius ${ }^{1}$, O. Dietle ${ }^{1}$ and G. Thimm ${ }^{4}$ \\ 1 Astronomisches Institut, Ruhr-Universität Bochum, D-44780 Bochum, Germany \\ 2 Astronomisches Institut, Universität Tübingen, Waldhäuser Str. 64, D-72076 Tübingen, Germany \\ 3 Astrofysisch Instituut, Vrije Universiteit Brussel, Pleinlaan 2, B-1050 Brussel, Belgium \\ 4 European Southern Observatory, Karl-Schwarzschild-Straße 2, D-85748 Garching, Germany
}

Received August 7; accepted September 25, 1995

\begin{abstract}
We present an atlas of high- $S / N$, high-resolution $\left(\Delta v \leq 6 \mathrm{~km} \mathrm{~s}^{-1}\right)$ data of Be star emission and shell profiles. We have collected profiles of $\mathrm{H} \alpha$ and of Fe II, mostly of the $\lambda 5317$ transition. These lines have been selected to provide measures for the overall emission strength and for the velocity field in these disks. We have collected data for 77 southern and equatorial programme stars, covering the period 1982-1993. This is the most comprehensive overview of profile shapes in Be disks. We propose a three-dimensional scheme in which most observed profiles can be classified. The parameters are i) inclination, ii) optical depth, and iii) the pattern of the velocity field. A search for short-term variability (timescales between five days and a few minutes) in six stars ended with negative result. Shortest observed timescale for variability is a few days for well-developed disks in binary systems (HR 1910, HR 2142).
\end{abstract}

Key words: atlas — stars: Be — lines: profiles

\section{Introduction}

Be stars are early-type non-supergiant stars surrounded by a cooler, disk-like circumstellar envelope. The envelope becomes apparent at optical wavelengths by hydrogen and metallic emission lines, and in the IR range by continuous bound-free and free-free thermal emission. The origin and structure of the circumstellar disk has been the aim of many studies since Struve (1931).

Since the disk is optically thin to continuous radiation in the visible range, most information about its structure can be gained from spectroscopy of its Doppler broadened emission lines. Among the most interesting physical parameters which can be investigated by emission line spectroscopy are:

- the envelope geometry (disk-like vs. ellipsoidal shell),

- its extension (radius, mass and emissivity distribution),

- its kinematics (Keplerian rotation vs. outflow),

- its density structure (hydrostatic equilibrium vs. windcompressed disk, density waves).

While the importance of studying emission lines has been recognized since long, the observational capabilities

Send offprint requests to: R.W. Hanuschik, Bochum address

${ }^{\star}$ Based on observations obtained at the European Southern Observatory, La Silla, Chile were quite limited as long as photographic plates were the standard detectors. Since the achievement of fast linear photoelectric devices in the past two decades, highresolution, high- $S / N$ spectroscopy has become possible, the only technique adequate for obtaining fully resolved line profiles free from instrumental effects.

Among the first steps towards such high-quality data were the atlases of Andrillat \& Fehrenbach (1982) and Andrillat (1983) who presented data for a large sample of northern and equatorial Be stars suffering only from insufficient $S / N$, and of Dachs et al. $(1981,1986)$ with a similarly large sample of southern and equatorial objects, measured at, however, insufficient resolution. Furthermore there exist three atlases in the infrared region (Andrillat et al. 1988, 1990, 1994), the first two at low, the last at medium resolution.

While these atlases represented a large step forward for Be star diagnostics, they still kept some important finestructure properties of emission line profiles hidden. This became apparent in the first high- $S / N$ and high-resolution atlases by Hanuschik $(1986,1987)$, Hanuschik et al. (1988, $=$ HKK88), Doazan et al. (1991), Slettebak et al. (1992, = S192) and Dachs et al. (1992). Good examples for such detection of important fine-structure are:

- the winebottle-type structure typical of emission line profiles in many low-to-intermediate inclination $\mathrm{Be}$ 
stars, formerly hidden in the flanks of low- $S / N$ data (Hanuschik 1986, HKK88);

- the detection of narrow emission peaks (of width $\leq 20$ $\mathrm{km} \mathrm{s}^{-1}$ ) in Fe II emission line profiles ("steeple"-type; Hanuschik 1987; Hanuschik et al. 1995a);

- the detection of an extremely narrow component in Fe II shell (absorption) lines (width $\leq 6 \mathrm{~km} \mathrm{~s}^{-1}$; Sect. 3.2 , this paper).

Apart from the quest for high spectral resolution, another goal of Be star spectroscopy has always been a good time coverage of the variability behaviour. The papers of Dachs et al. $(1981,1986)$ were the first to present a longterm overview of the emission behaviour in a larger sample of Be stars. In the meanwhile they represent important cornerstones for long-term variability studies. Other such studies, like the atlases mentioned above, form snapshots only.

We feel that a synoptic time-resolved study of highresolution, high- $S / N$ emission line profiles of Be stars is both desirable and still missing. To present such study is the purpose of the present paper. We provide a collection of data for 77 southern and equatorial Be stars, most of which have not yet been published. Our data cover more than a full decade for many objects. We are convinced that this number is sufficient to cover all important phenomena connected with Be stars and visible in optical emission lines.

In a forthcoming paper we will analyze these data, with emphasize on the long-term variability behaviour of Be star disks.

In Sect. 2 we present a short overview of the selected lines and stars, and of the measurements and reduction procedure. The atlas with the full set of profiles is presented in Sect. 3. A three-dimensional classification scheme for Be star profiles is devised in Sect. 4. Finally we present a search for short-term variability in Sect. 5 .

\section{Data}

\subsection{Selection of lines}

A combined study of high-opacity Balmer lines and lowopacity $\mathrm{Fe}$ II lines is very efficient in distinguishing the different shaping and broadening effects in emission line profiles due to kinematics and radiative transfer.

$\mathrm{H} \alpha$ measurements are mainly valuable as indicators for overall emission strength and its variability, and for asymmetry of the density distribution.

Fe II lines are optically thin and unperturbed by photospheric features. They reflect the pure kinematical broadening function in the emitting part of the Be disk (if thermal and turbulent motions are negligible) and offer highly valuable insight into processes within the disk.

We have selected profiles of

- $\mathrm{H} \alpha \lambda 6562.8$, and

- Fe II $\lambda 5316.6$.
During the 1985 Feb./March and the 1989 March runs, occasionally the Fe II $\lambda 5169$ line was measured instead.

During some of the runs, $\mathrm{H} \beta, \mathrm{H} \gamma$ and $\mathrm{He}$ I lines were measured in addition. These profiles have not been included in this study to avoid overcrowding. They can be found in Hanuschik (1987, together with some more Fe II lines), HKK88, S192 and Dachs et al. (1992).

\subsection{Measurements and reduction}

Most data have been measured at ESO's 1.4m Coudé Auxiliary Telescope, using the Coudé Echelle Spectrograph and a reticon (until 1987) or a CCD (as of 1988), resp., as detector. The spectrograph operated at high resolution, $R=\lambda / \Delta \lambda \geq 50000$, corresponding to $\Delta \lambda \leq 0.13 \AA$ at $\mathrm{H} \alpha$ and $\Delta v \leq 6 \mathrm{~km} \mathrm{~s}^{-1}$. This is sufficient to fully resolve all intrinsic structure of the $\mathrm{H} \alpha$ profiles (thermal velocity about $20 \mathrm{~km} \mathrm{~s}^{-1}$ at $10^{4} \mathrm{~K}$ ), and also practically all intrinsic structure of the Fe II profiles. The signal-to-noise ratio $(S / N)$ is usually in the range 100-1000 (the higher value being representative for the brightest $\mathrm{H} \alpha$ profiles). An overview of the measuring campaigns and the observers involved is contained in Table 1.

The reduction of the data has been standard. The steps were bias subtraction, division by the normalized flat field, extraction in case of CCD data (integration perpendicular to the dispersion of all pixels in which $S \geq N$ ), normalization to the stellar continuum, calibration to heliocentric radial velocity scale, and correction for telluric absorption lines (except for the 1982 CAT data and those of Sl92, see below). This latter step was performed using a mask provided by an almost featureless comparison spectrum (mostly of the O5Ia star $\zeta$ Pup). More details can be found in Hanuschik (1986) and Dachs et al. (1992).

Where appropriate, we have included for comparison $\mathrm{H} \alpha$ and some $\mathrm{H} \beta$ data from the atlas of Sl92. These data are usually not fully resolved, $R=15000$, but have been included here in order to fill the time record.

\subsection{Programme stars}

Our programme stars are collected in Table 2. They have been selected by apparent brightness and overlap with older data bases like the atlases of Dachs et al. (1981, 1986). Furthermore an attempt has been made to include at least one measurement of as many southern and equatorial stars from the list of Slettebak (1982) as possible, motivated by the consideration that a study of Be disk behaviour containing as much information as possible should include a cross-section (many stars measured once) as well as a longitudinal section (few stars measured frequently).

Table 3 contains the approximate dates of measurements. The periods are coded following Table 1. The exact dates can be found as labels in the profile figures (see below). 
Table 1. Observing campaigns at ESO $1.4 \mathrm{~m} \mathrm{CAT}$

\begin{tabular}{lllll}
\hline No. & Date (UT) & Observer & Detector & Source of publication \\
\hline$\# 1$ & $\begin{array}{l}\text { 1982 Aug 15-Sep 15 } \\
\text { (13 nights) }\end{array}$ & J.R. Kozok & Reticon & Hanuschik et al. 1988 \\
\#2a & 1985 Jan 01-04 & R.W. Hanuschik & Reticon & Hanuschik (1986, 1987) \\
$\#$ 2b & 1985 Feb 27-Mar 03 & R.W. Hanuschik & Reticon & Hanuschik (1986, 1987) \\
$\# 3$ & 1987 Feb 07-13 & J. Dachs & Reticon & Dachs et al. (1992) \\
$\# 4 a$ & 1988 Dec 03-04 & G. Thimm & CCD & unpublished \\
$\# 4 b$ & 1989 Mar 02-05 & G. Thimm & CCD & unpublished \\
$\# 5$ & 1989 Sep 26-Oct 01 & R.W. Hanuschik & CCD & unpublished \\
$\# 6 a$ & 1992 Jan 11-13 & W. Hummel, R.W. Hanuschik & CCD & unpublished \\
$\# 6 b$ & 1992 Mar 24-25 & W. Hummel & CCD & unpublished \\
$\# 7 a$ & 1993 Apr 18 & R.W. Hanuschik & CCD & unpublished \\
$\# 7 b$ & 1993 Sep 08-09 & R.W. Hanuschik & CCD & unpublished \\
some profiles are added from the following campaigns: & \multirow{2}{*}{ Sl92 } \\
S1 & 1989 Jan 06-09 & A. Slettebak & $a$ & Sl92 \\
S2 & 1989 Oct 06-09 & A. Slettebak & & \\
\hline a KPNO Coudé Feed Telescope, CCD & &
\end{tabular}

${ }^{a}$ KPNO Coudé Feed Telescope, CCD

\section{The atlas}

\subsection{The profiles}

In Figs. 5-82, we show the full set of our profiles for 77 southern and equatorial Be and shell stars from 19821993.

For each star with multiple measurements, we show three panels which essentially contain the same information but emphasize different aspects of variability. In panel a, all profiles are plotted on top of each other which pronounces variations in radial velocity and line profile shape. Panel b provides an overview of the overall emission strength behaviour, e.g. variations of central intensity. In panel c, both aspects (profile shape and emission strength) are combined. This latter plot is intended as demonstration of the degree of constancy (of the whole profile or parts thereof). In case of strongly variable profiles, or many measurements, this panel may become a bit overcrowded and is only thought as visualization of variability (or constancy).

In panels a and b, profiles of $\mathrm{H} \alpha$ and Fe II $\lambda 5317$ (or Fe II $\lambda 5169$, if noted) are plotted together. These measurements are quasi-simultaneous, i.e. separated by at most 2-3 days (usually one day).

Since the Fe II lines are comparably faint (peak flux $\leq$ $1.2 F_{\mathrm{c}}$ ), their profiles have been blown up according to

$$
F^{\prime}(\lambda) / F_{\mathrm{c}}=\left(F(\lambda) / F_{\mathrm{c}}-1\right) \cdot k+1,
$$

where $F(\lambda)$ denotes the measured, and $F^{\prime}(\lambda)$ the displayed flux, resp. The expansion factor $k$ is in the range $5-20$ and displayed in the figures.

If the Fe II region has been measured, but no emission has been found, the pure stellar continuum is plotted since this negative result about Fe II emission is as valuable as a positive one, e.g. for further investigations of the ionization conditions in the disk.

\subsection{Individual stars}

We will emphasize here new results about the most interesting objects. Overviews about the history of objects already observed earlier may be found, e.g., in Slettebak (1982) or the atlases cited in the Introduction.

HR $1180=28$ Tau $=$ Pleione. This star is famous for its phase transitions between $\mathrm{Be}$ and Be-shell spectrum. It is the only example in our atlas showing such behaviour. We have caught the star in its most recent transition where it lost its shell characteristics at some time between 1989 (when the $\mathrm{H} \alpha$ profile still shows a deep central depression) and 1993 when the Fe II profile clearly exhibits pure emission characteristics (see Fig. 9). The whole transition, however, has been quite a smooth process lasting for about a decade. The depth of the $\mathrm{H} \alpha$ depression has continuously decreased since 1982 , while the $\mathrm{H} \alpha$ equivalent width has increased. Furthermore, in 1993 the profile has become asymmetric and presumably shows onset of cyclic $V / R$ variability.

HR $1423=28$ Eri. This star has lost its disk almost entirely between 1989 and 1992 (Fig. 10). The 920111 profile of $\mathrm{H} \alpha$ looks quite bizarre since it shows, apart from its double peak, secondary ondulations in the wings (Fig. 10d) which are unlike anything visible in Figs. 1 or 2 . These are likely to be indicators of a strongly distorted velocity field. The distortion may either be linked to the decay mechanism or to a highly inhomogeneous density 
Table 2. Programme stars

\begin{tabular}{|c|c|c|c|c|}
\hline HR & Name & MK type ${ }^{a}$ & $\begin{array}{l}v \sin i^{a} / \\
\mathrm{km} \mathrm{s}^{-1}\end{array}$ & $\begin{array}{c}\text { Profile } \\
\text { code }^{c}\end{array}$ \\
\hline 472 & $\alpha$ Eri & $\mathrm{B} 4 \mathrm{~V}(\mathrm{e})$ & 225 & $(\mathrm{~L}) 1$ \\
\hline 1142 & $17 \mathrm{Tau}$ & B6 IIIe & 180 & $(\mathrm{~L}) 1$ \\
\hline 1156 & $23 \mathrm{Tau}$ & B6 IV(e) & 280 & $\mathrm{~L}-\mathrm{H} 1$ \\
\hline 1165 & $\eta \mathrm{Tau}$ & B7 IIIe & 140 & L:1 \\
\hline 1180 & $28 \mathrm{Tau}$ & B8 Ve-sh & 320 & $\mathrm{E} 1 \rightarrow \mathrm{H} 2$ \\
\hline 1423 & 28 Eri & B1 Ve & 230 & $\mathrm{~L}-\mathrm{H} 1$ \\
\hline 1508 & 56 Eri & $\mathrm{B} 2 \mathrm{Ve}$ & 180 & L1 \\
\hline 1660 & $105 \mathrm{Tau}$ & B3 Ve & 200 & L2 \\
\hline 1679 & $\lambda$ Eri & B2 IIIe & 220 & $(\mathrm{~L}) 1$ \\
\hline 1772 & & $\mathrm{~B} 5 \mathrm{IVe}^{b}$ & $\ldots$ & E1 \\
\hline 1789 & 25 Ori & $\mathrm{B} 1 \mathrm{Ve}$ & 320 & L-H1 \\
\hline 1858 & $120 \mathrm{Tau}$ & B1.5 IVe & 210 & $\mathrm{~L}-\mathrm{H} 1$ \\
\hline 1910 & $\zeta \mathrm{Tau}$ & B1 IVe-sh & 220 & $\mathrm{E} 1 \rightarrow 2$ \\
\hline 1934 & $\omega$ Ori & B2 IIIe & 160 & L1 \\
\hline 1956 & $\alpha \mathrm{Col}$ & B7 IVe & 180 & L1 \\
\hline 1961 & & $\mathrm{~B} 2.5 \mathrm{Ve}^{b}$ & $\ldots$ & $\mathrm{L} 2$ \\
\hline 2142 & & B2 IVe & 350 & $\ldots$ \\
\hline 2170 & & $\mathrm{~B} 5 \mathrm{Ve}$ & 220 & L1 \\
\hline 2284 & & B1.5 IVe & 200 & L2: \\
\hline 2343 & $\nu \mathrm{Gem}$ & B6 IVe & 170 & $\mathrm{E} 2$ \\
\hline 2356 & $\beta^{1}$ Mon & B4 Ve-sh & 300 & $\mathrm{H} 2$ \\
\hline 2492 & $10 \mathrm{CMa}$ & B2 IIIe & 200 & L1 \\
\hline 2538 & $\kappa \mathrm{CMa}$ & B2 IVe & 220 & L2 \\
\hline 2545 & & $\mathrm{~B} 6 \mathrm{IVe}+\mathrm{A}$ & 220 & $\ldots$ \\
\hline 2690 & & B2 IVe & 200 & $\mathrm{~L}: 1$ \\
\hline 2745 & $27 \mathrm{CMa}$ & B3 III(e)-sh & 150: & abs \\
\hline 2749 & $\omega \mathrm{CMa}$ & $\mathrm{B} 2.5 \mathrm{Ve}$ & 80 & L1 \\
\hline 2787 & & $\mathrm{~B} 2 \mathrm{Ve}$ & 220 & L1 \\
\hline 2825 & & B3 Ve & $\leq 40$ & $\mathrm{P} 1^{d}$ \\
\hline 2845 & $\beta \mathrm{CMi}$ & $\mathrm{B} 8 \mathrm{Ve}$ & 245 & $\mathrm{~L}-\mathrm{H} 1$ \\
\hline 2855 & & B0.5 IVe & 280 & $\ldots$ \\
\hline 2911 & & $\mathrm{~B} 3 \mathrm{Ve}$ & 245 & $\mathrm{H} 1$ \\
\hline 2921 & & B3 IV & 230 & $\mathrm{~L}: 1$ \\
\hline 3034 & $o$ Pup & B1 IVe & 320 & $\mathrm{~L}-\mathrm{H} 2$ \\
\hline 3135 & & B2.5 Ve & 140 & L1 \\
\hline 3186 & & $\mathrm{~B} 2.5 \mathrm{Vn}^{b}$ & $292^{b}$ & $\mathrm{H}: 1$ \\
\hline 3237 & & B1.5 IVe & 115 & $\mathrm{P}-\mathrm{L} 2$ \\
\hline
\end{tabular}

Table 2. continued

\begin{tabular}{|c|c|c|c|c|}
\hline $\mathrm{HR}$ & Name & MK type $^{a}$ & $\begin{array}{l}v \sin i^{a} / \\
\mathrm{km} \mathrm{s}^{-1}\end{array}$ & $\begin{array}{c}\text { Profile } \\
\text { code }\end{array}$ \\
\hline 3498 & & $\mathrm{~B} 2.5 \mathrm{~V}(\mathrm{e})$ & 240 & (L)1 \\
\hline 3858 & & $\mathrm{~B} 5 \mathrm{Ve}$ & 260 & L1 (2?) \\
\hline 3946 & & $\mathrm{~B} 4 \mathrm{Ve}^{b}$ & $220^{b}$ & L1 (2?) \\
\hline 4009 & & B2 IVe & 220 & L2 \\
\hline 4037 & $\omega$ Car & B8 IIIe-sh & 220 & $\mathrm{H}-\mathrm{E} 1$ \\
\hline 4123 & & B9 IVe & 250 & $\mathrm{~L}-\mathrm{H} 1$ \\
\hline 4140 & p Car & $\mathrm{B} 4 \mathrm{Ve}$ & 250 & L2 \\
\hline 4621 & $\delta$ Cen & B2 IVe & 220 & L2 \\
\hline 4823 & & B6 IVe & 250 & H1 \\
\hline 4830 & & B1 IIIe & 300 & L-H1 \\
\hline 4899 & $\mu^{2} \mathrm{Cru}$ & B5 IVe & 220 & $\mathrm{~L}-\mathrm{H} 1$ \\
\hline 4930 & & B2 IIIe & 300 & $\mathrm{~L}-\mathrm{H} 2$ \\
\hline 5193 & $\mu$ Cen & B2 IV-Ve & 155 & L1 \\
\hline 5223 & & B2 IIIep & 70 & P1 (2?) \\
\hline 5316 & & $\mathrm{~B} 4 \mathrm{Ve}$ & 300 & H1 \\
\hline 5440 & $\eta$ Cen & B2 IV(e) & 350 & $\mathrm{H}-\mathrm{E} 1$ \\
\hline 5551 & $\theta$ Cir & B3 V(e) & 100 & (L2?) \\
\hline 5661 & & $\mathrm{~B} 0 \mathrm{~V}$ & 130 & abs \\
\hline 5730 & $\kappa^{1} \mathrm{Aps}$ & B3 IVe & $250-350$ & $\mathrm{H}-\mathrm{E} 1$ \\
\hline 5941 & $48 \mathrm{Lib}$ & B3 IV e-sh & 400 & E2 \\
\hline 6118 & $\chi \mathrm{Oph}$ & $\mathrm{B} 1.5 \mathrm{Ve}$ & 140 & $\mathrm{P} 2^{e}$ \\
\hline 6304 & & B2 IVne ${ }^{b}$ & $201^{b}$ & $\mathrm{~L}-\mathrm{H} 2 ?$ \\
\hline 6397 & & O7.5 IIIe & 115 & $\ldots$ \\
\hline 6451 & $\iota$ Ara & B2.5 IVe & 320 & H2 \\
\hline 6510 & $\alpha$ Ara & B3 Ve & 250 & $\mathrm{~L}-\mathrm{H} 1$ \\
\hline 6712 & $66 \mathrm{Oph}$ & B2 IV-Ve & 240 & $\mathrm{~L} 2$ \\
\hline 6819 & & B3 III(e) & 50 & (L) 1 \\
\hline 6929 & & $\mathrm{~B} 2 \mathrm{IVpe}^{b}$ & $\ldots$ & $\ldots$ \\
\hline 7249 & & $\mathrm{~B} 2 \mathrm{~V}(\mathrm{e})$ & 120 & L1 (2?) \\
\hline 7415 & & B6 e-sh & 300 & E2 \\
\hline 7789 & $25 \mathrm{Vul}$ & B6 IVe & 200 & L1 \\
\hline 8260 & $\epsilon$ Cap & B3 IIIe & 250 & E1 \\
\hline 8402 & $o$ Aqr & B7 IIIe-sh & 300 & E1 \\
\hline 8438 & $25 \mathrm{Peg}$ & $\mathrm{B} 6 \mathrm{Ve}$ & 150 & L1 \\
\hline 8520 & $31 \mathrm{Peg}$ & $\mathrm{B} 1.5 \mathrm{Ve}$ & 100 & L1 \\
\hline 8539 & $\pi$ Aqr & B1 III-IVe & 300 & (H2?) \\
\hline 8628 & $\epsilon \mathrm{PsA}$ & B7 IVe & 180 & (L2?) \\
\hline 8773 & $\beta \mathrm{Psc}$ & B5 Ve & 100 & $\mathrm{P} 1$ \\
\hline 9076 & $\epsilon$ Tuc & $\mathrm{B} 8 \mathrm{~V}$ & 280 & $(\mathrm{H}) 1$ \\
\hline 9098 & 2 Cet & $\mathrm{B} 9.5 \mathrm{Vn}^{b}$ & $186^{b}$ & (L) 1 \\
\hline
\end{tabular}


distribution with a high-density part producing electron scattering wings, and a low-density region without Thomson scattering. Since the ondulations are quite sharply limited, these regions may even be separated in space. Whatever the true origin for the unique profile shape is, it must be a transient phenomenon since neither before nor after 1992 such shape has been observed.

HR 1508 = 56 Eri. This star shows conspicuous line profile variations which appear as a change in typical line width (cf. the profiles in 1988 and 1982: Figs. 11c, d), with the whole profile remaining highly symmetric throughout the observed period. The $1982 \mathrm{H} \alpha$ profile shows textbook examples of very pronounced winebottle inflections.

HR 1660. The profile asymmetry slowly changes on a timescale of about 10 years, accompanied by typical steeple-shape in the Fe II profile (Fig. 12).

HR $1679=\lambda$ Eri. This star is a prototype example for flickering emission. $\lambda$ Eri has shown this behaviour in 1980-1983 (Barker 1986; Dachs et al. 1986) as well as in the more recent past. It appears to be a twin of $\mu$ Cen (Hanuschik et al. 1993) and is known to exhibit a complex emission behaviour in the He I lines (Smith 1989). Its last outburst was reported in August 1994 (Štefl 1994).

HR $1910=\zeta$ Tau. The behaviour of the $\mathrm{H} \alpha$ line is quite complex: starting from a minimum emission strength and symmetric shape in 1982, the profile gets brighter till 1993 and starts cyclic $V / R$ activity in about 1989 . The estimated cycle duration is about $6-7$ years, with a $V=R$ transition between 1992 February and November (Guo 1994). The profiles look smooth, except for the 1982 set, see Fig. 17d. Since $\zeta$ Tau is a well-known binary $\left(P_{\text {orb }}=132.91^{\mathrm{d}}\right.$, Pols et al. 1991), the 1982 finestructure may indicate interaction or disk distortion.

Alternatively, the finestructure could indicate an unstable state of the disk, e.g. a density wave with increasing amplitude getting non-linear.

HR 2142. Its binary nature with $P_{\text {orb }}=80.860$ days has been determined by Peters (1983). We clearly see the rare case of an interacting binary Be star of the same kind of behaviour as $\phi$ Per (Gies et al. 1993). We show in Fig. 23 the whole set of $\mathrm{H} \alpha$ profiles collected at the CAT over 11 years, phase binned with the above period. Although some secular variability in strength exists, the basic shape of the $\mathrm{H} \alpha$ profile is repeated, even after 50 orbital periods.

HR $2343=\nu$ Gem. From all stars in our sample showing cyclical $V / R$ variability, this star has the faintest emission (Fig. 25). We estimate its cycle duration to about 5 years, but it might also be that we observe orbital modulation with a much shorter period since $\nu$ Gem is a binary Be star $\left(P_{\text {orb }}=40.198^{\mathrm{d}}\right.$, Jarad et al. 1989). Note also the very narrow and untypical Fe II absorption and emission in 1992 (Fig. 25).

HR $2538=\kappa$ CMa. Since 1982, this star has been observed to show an asymmetric $\mathrm{H} \alpha$ profile and steepletype Fe II profile (Fig. 28). Contrary to all other such stars in our survey, it has not shown any considerable variation of this asymmetry, thus making it the star with the longest cycle duration in our sample $(P \gg 20 \mathrm{yr})$.

HR $2749=\omega$ CMa. This star has shown development of broad Thomson wings in 1987 and 1993 (cf. Fig. $32 d)$.

HR 2825. Like $\omega$ CMa, this star shows Thomson wing variability, with broad wings in 1992/93 and a narrow profile in 1987-1989 (Fig. 34). These variations indicate a considerable change in electron density. Note also the extreme anticorrelation between the width and strength of the Fe II emission and the strength of $\mathrm{H} \alpha$ emission (Fig. 34d) which is also observed in HR 5223 (Fig. 56d).

HR 3034 = o Pup. The line profiles show cyclical $V / R$ variability, with $P \approx 8$ years, as well as some shortterm variability in 1992 January-March (Fig. 39).

HR 3237. This star is a typical $V / R$ variable star with steeple-type Fe II profiles at certain epochs of its cycle (present duration: 9 years, Hanuschik et al. 1995a; Fig. 42). Emission of this pole-on star is among the strongest in our sample.

HR 4009. A quite pronounced $V / R$ activity has started between 1989 and 1992, with a very narrow steeple-shaped Fe II profile in 1992 (Fig. 46).

HR $4140=$ p Car. This star exhibits cyclical $V / R$ variability, with the $890304 \mathrm{Fe}$ II profile being considerably asymmetric (Fig. 49). The cycle duration is not yet known.

HR 4621 $=\delta$ Cen. We observe prototype $V / R$ variability, with $P \approx 10 \mathrm{yr}$ (Hanuschik et al. 1995a), connected with typical steeple-shaped Fe II profiles in 1985 and 1989 (Fig. 50).

HR 4930. This star shows variability with an approximate cycle duration of about 10 years, with extreme asymmetry in $1987(V<R)$ and $1992(V>R)$, and approximate symmetry in 1993.

HR $5193=\mu$ Cen. This star is one of the few well studied southern Be stars and has become famous for its loss of disk emission in 1972-1976 (Peters 1979), and its flickering emission activity since then (Baade et al. 1988, Hanuschik et al. 1993). Since 1993, this star is building up a new permanent disk (Fig. 55).

HR 5223. This pole-on star shows weak $V / R$ variability and variability of strength of the Thomson scattering wings in $\mathrm{H} \alpha$ and the Fe II width (Fig. 56).

HR $5551=\theta$ Cir. This star behaves atypical of Be stars in the way it shows irregular line profiles with shortterm variability (Fig. 59). Its Fe II profiles are variable as well, with a very broad profile in 1993 as compared to the 1989 and 1992 profiles (Fig. 59).

HR $5941=48$ Lib. Known as $V / R$ variable star since long, its present cycle duration is 9yr (Hanuschik et al. 1995a and references therein). Its $\mathrm{H} \alpha$ and Fe II shell troughs are the deepest ones in our sample $\left[F / F_{\mathrm{c}}(\mathrm{H} \alpha)=\right.$ 0.11 in $1985, F / F_{\mathrm{c}}(\mathrm{Fe}$ II $)=0.095$ in 1993, Fig. 63$)$. 
Table 3. Dates of measurements ( $\mathrm{H} \alpha$ and/or Fe II)

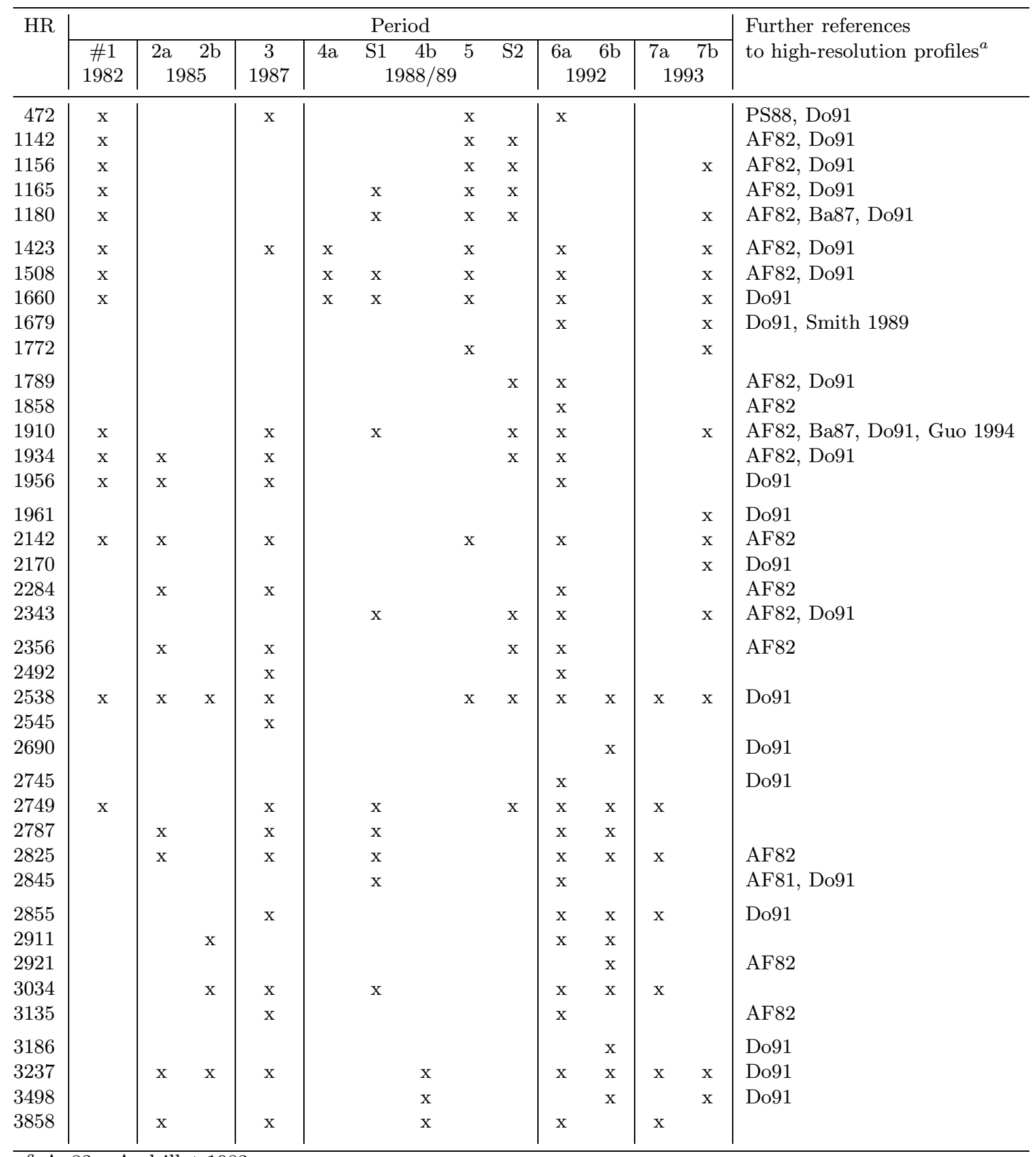

\footnotetext{
${ }^{a}$ An83 - Andrillat 1983

Ba87 - Ballereau et al. 1987

Do91 - Doazan et al. 1991

PS88 - Porri \& Stalio 1988
} 
Table 3. continued

\begin{tabular}{|c|c|c|c|c|c|c|c|c|c|c|c|c|c|c|}
\hline \multirow[t]{2}{*}{$\mathrm{HR}$} & \multicolumn{13}{|c|}{ Period } & \multirow{2}{*}{$\begin{array}{l}\text { Further references } \\
\text { to high-resolution profiles }{ }^{a}\end{array}$} \\
\hline & $\begin{array}{c}\# 1 \\
1982\end{array}$ & $\begin{array}{l}2 \mathrm{a} \\
\quad 198\end{array}$ & $\begin{array}{l}2 \mathrm{~b} \\
85\end{array}$ & $\begin{array}{c}3 \\
1987\end{array}$ & $4 a$ & & $\begin{array}{c}4 \mathrm{~b} \\
88 / 8\end{array}$ & 5 & $\mathrm{~S} 2$ & $\begin{array}{r}6 \mathrm{a} \\
1\end{array}$ & $\begin{array}{l}6 \mathrm{~b} \\
92\end{array}$ & \multicolumn{2}{|c|}{$\begin{array}{c}7 \mathrm{a} \quad 7 \mathrm{~b} \\
1993\end{array}$} & \\
\hline 3946 & & $\mathrm{x}$ & & $\mathrm{x}$ & & & $\mathrm{x}$ & & & $\mathrm{x}$ & $\mathrm{x}$ & $\mathrm{x}$ & & \\
\hline 4009 & & $\mathrm{x}$ & & $\mathrm{x}$ & & & $\mathrm{x}$ & & & $\mathrm{x}$ & $\mathrm{x}$ & $\mathrm{x}$ & & Do91 \\
\hline 4037 & & & & & & & & & & & & $\mathrm{x}$ & & Do91 \\
\hline 4123 & & & & & & & & & & & $\mathrm{x}$ & $\mathrm{x}$ & & AF82, Do91 \\
\hline 4140 & & $\mathrm{x}$ & $\mathrm{x}$ & $\mathrm{x}$ & & & $\mathrm{x}$ & & & $\mathrm{x}$ & $\mathrm{x}$ & $\mathrm{x}$ & & PS88, Do91 \\
\hline 4621 & & $\mathrm{x}$ & $\mathrm{x}$ & $\mathrm{x}$ & & & $\mathrm{x}$ & & & $\mathrm{x}$ & $\mathrm{x}$ & $\mathrm{x}$ & & Baade 1987, Do91 \\
\hline 4823 & & & & $\mathrm{x}$ & & & $\mathrm{x}$ & & & $\mathrm{x}$ & $\mathrm{x}$ & $\mathrm{x}$ & & Do91 \\
\hline 4830 & & $\mathrm{x}$ & & $\mathrm{x}$ & & & $\mathrm{x}$ & & & $\mathrm{x}$ & & & & Do91 \\
\hline 4899 & & & & $\mathrm{x}$ & & & & & & & & & & \\
\hline 4930 & & & & $\mathrm{x}$ & & & & & & $\mathrm{x}$ & $\mathrm{x}$ & $\mathrm{x}$ & $\mathrm{x}$ & \\
\hline 5193 & & & $\mathrm{xx}^{b}$ & $\mathrm{x}$ & & & $\mathrm{x}$ & & & $\mathrm{x}$ & $\mathrm{x}$ & $\mathrm{x}$ & $\mathrm{x}$ & $\begin{array}{l}\text { Peters 1986, Baade et al. 1988, } \\
\text { Do91, Ghosh et al. } 1991\end{array}$ \\
\hline 5223 & & & $\mathrm{x}$ & $\mathrm{x}$ & & & $\mathrm{x}$ & & & $\mathrm{x}$ & $\mathrm{x}$ & $\mathrm{x}$ & $\mathrm{x}$ & Do91 \\
\hline 5316 & & & $\mathrm{x}$ & $\mathrm{x}$ & & & $\mathrm{x}$ & & & & $\mathrm{x}$ & & & Do91 \\
\hline 5440 & $\mathrm{x}$ & & & $\mathrm{x}$ & & & $\mathrm{x}$ & & & $\mathrm{x}$ & & $\mathrm{x}$ & & Do91 \\
\hline 5551 & $\mathrm{x}$ & & & $\mathrm{x}$ & & & $\mathrm{x}$ & & & $\mathrm{x}$ & $\mathrm{x}$ & $\mathrm{x}$ & $\mathrm{x}$ & \\
\hline 5661 & & & & & & & & & & & $\mathrm{x}$ & & & \\
\hline 5730 & & & & & & & & & & & $\mathrm{x}$ & & & Do91 \\
\hline 5941 & & & $\mathrm{x}$ & $\mathrm{x}$ & & & & & & & $\mathrm{x}$ & $\mathrm{x}$ & $\mathrm{x}$ & $\mathrm{AF} 82$ \\
\hline 6118 & & & $\mathrm{x}$ & $\mathrm{x}$ & & & $\mathrm{x}$ & $\mathrm{x}$ & & & $\mathrm{x}$ & $\mathrm{x}$ & $\mathrm{x}$ & $\mathrm{AF} 82$ \\
\hline 6304 & & & & & & & & $\mathrm{x}$ & & & $\mathrm{x}$ & $\mathrm{x}$ & $\mathrm{x}$ & Do91 \\
\hline 6397 & $\mathrm{x}$ & & & & & & & $\mathrm{x}$ & & & $\mathrm{x}^{c}$ & $\mathrm{x}^{c}$ & & \\
\hline 6451 & & & $\mathrm{x}$ & $\mathrm{x}$ & & & & $\mathrm{x}$ & & & $\mathrm{x}$ & $\mathrm{x}$ & $\mathrm{x}$ & Do91 \\
\hline 6510 & $\mathrm{x}$ & & $\mathrm{x}$ & $\mathrm{x}$ & & & & $\mathrm{x}$ & & & $\mathrm{x}$ & & $\mathrm{x}$ & \\
\hline 6712 & & & & & & & & $\mathrm{x}$ & $\mathrm{x}$ & & $\mathrm{x}$ & $\mathrm{x}$ & $\mathrm{x}$ & AF82, Peters 1988, Do91 \\
\hline 6819 & & & & & & & & $\mathrm{x}$ & & & & & & Do91 \\
\hline 6929 & & & & & & & & $\mathrm{x}$ & & & $\mathrm{x}$ & $\mathrm{x}$ & & \\
\hline 7249 & $\mathrm{x}$ & & & & & & & $\mathrm{x}$ & & & $\mathrm{x}$ & $\mathrm{x}$ & & Do91 \\
\hline 7415 & & & & & & & & & & & & $\mathrm{x}$ & $\mathrm{x}$ & Ba87, Do91 \\
\hline 7789 & & & & & & & & & $\mathrm{x}$ & & & & $\mathrm{x}$ & An83, Do91 \\
\hline 8260 & $\mathrm{x}$ & & & & & & & $\mathrm{x}$ & & & & $\mathrm{x}$ & $\mathrm{x}$ & An83, PS88, Do91 \\
\hline 8402 & $\mathrm{x}$ & & & & & $\mathrm{x}$ & & $\mathrm{x}$ & & & & $\mathrm{x}$ & $\mathrm{x}$ & An83, Do91 \\
\hline 8438 & $\mathrm{x}$ & & & & & & & $\mathrm{x}$ & & & & & $\mathrm{x}$ & AF82, Do91 \\
\hline 8520 & $\mathrm{x}$ & & & & & $\mathrm{x}$ & & $\mathrm{x}$ & & & & $\mathrm{x}$ & $\mathrm{x}$ & AF82 \\
\hline 8539 & $\mathrm{x}$ & & & & & $\mathrm{x}$ & & $\mathrm{x}$ & & & & $\mathrm{x}$ & $\mathrm{x}$ & AF82, Do91 \\
\hline 8628 & $\mathrm{x}$ & & & & & & & $\mathrm{x}$ & & & & $\mathrm{x}$ & & Do91 \\
\hline 8773 & $\mathrm{x}$ & & & & & $\mathrm{x}$ & & $\mathrm{x}$ & & & & & $\mathrm{x}$ & AF82, Do91 \\
\hline 9076 & $\mathrm{x}$ & & & & & & & $\mathrm{x}$ & & & & & & \\
\hline 9098 & & & & & $\mathrm{x}$ & & & & & & & & & \\
\hline
\end{tabular}

HR $6118=\chi$ Oph. Its profiles show a rare three peak appearance due to strongly developed winebottle-type shoulders (Fig. 62). We also observe a weakly developed $V / R$ variability with a still undetermined period of certainly more than $15-20 \mathrm{yr}$.

HR 6397. The rather odd profile of this O7.5e star in 1989 (Fig. 65) may indicate that in Oe-type stars kine- matics in optical emission disks are different from those in ordinary Be stars.

HR $6712=66$ Oph. This star has been observed to suddenly start $V / R$ variability in the course of 1988 , associated with the occurrence of prototype steeple-shaped Fe II line profiles (Hanuschik et al. 1995a, b; Fig. 68). Its present cycle duration is 5 years. 
HR 6929. This star, with moderately strong emission in 1982, has lost its disk until 1989 (Fig. 70). It presently shows flickering emission, very similar to $\mu$ Cen or $\lambda$ Eri.

HR $8402=$ o Aqr. This shell star, with very stable emission strength over 20 years, is remarkable since it shows an extremely narrow shell core in the Fe II lines (Fig. 75d). With a half-width of $6 \mathrm{~km} \mathrm{~s}^{-1}$ the narrow Fe II feature in 1989 is unresolved and forms the most narrow absorption feature from a Be star disk in our sample. It indicates disk matter in front of the star at considerable distance $(5-10$ stellar radii) being in almost perfect Keplerian rotation (radial component $\ll 6 \mathrm{~km} \mathrm{~s}^{-1}$ ).

HR $8539=\pi$ Aqr. With short-term profile variability in 1982 (Fig. 78), this star is a candidate interacting binary system, though it is not listed in Pols et al. 1991.

HR $8773=\beta$ Psc. This is another star with Thomson wing variability (Fig. 80).

\section{Line profile shapes}

Despite the apparently wild fluctuations in emission line shape, it is possible to arrange most observed line profiles into a rather simple scheme. There seem to be just three parameters: i) inclination $i$, ii) optical depth $\tau$, and iii) kinematics. Firstly we will demonstrate the effect of the first two parameters.

\subsection{Inclination and optical depth}

All lines are expected to be shaped by the influence of large-scale Doppler (kinematical) broadening,

$$
\Delta v_{\text {kin }}=v_{\mathrm{K}} \sin i
$$

where $v_{\mathrm{K}}$ denotes the Keplerian velocity at the stellar radius (= inner edge of the disk), and $i$ the inclination angle. $v_{\mathrm{K}}$ is of order $400-500 \mathrm{~km} \mathrm{~s}^{-1}$. It is natural to arrange line profiles along a $v \sin i$ sequence where $v \sin i$ is the stellar projected rotational velocity which measures the inclination if $v$ is known (or assumed to be uniform in all Be stars which is done here).

Emission lines are also broadened by thermal broadening $\Delta v_{\text {th }}$. This is of the order of $20 \mathrm{~km} \mathrm{~s}^{-1} / \sqrt{A}$ (with the atomic weight $A$ ) and can be neglected here.

The kinematical broadening becomes also manifest as small-scale process in thin disks, as shear broadening. This effect is caused by velocity shear between the upper and the lower intersection point of a certain line-of-sight and the disk (Horne \& Marsh 1986). The shear increases with inclination according to

$$
\Delta v_{\mathrm{sh}}=\Delta v_{\mathrm{th}} \cdot \frac{3}{4} \sin i \tan i \sin 2 \varphi
$$

( $\varphi$ is the azimuthal angle within the disk). For $i>60^{\circ}$, $\Delta v_{\text {sh }}$ may exceed the thermal broadening $\Delta v_{\text {th }}$ in certain directions. This causes a non-isotropic line absorption coefficient resulting in a deep V-shaped central depression.
This effect is even important for optically thin Fe II lines with their small thermal width.

In addition, optically thick lines, and especially $\mathrm{H} \alpha$, are shaped by other broadening mechanisms induced by radiative transfer. (We will use in the following the term optical depth, $\tau$, for the vertical optical depth at the innermost disk radius.)

The most important contribution to optically thick line shaping in disks is broadening by non-coherent scattering (NSB). It is caused by the effect that for optically thick lines, deeper layers of the disk become visible in the wings of the local absorption profile than in the core (Avrett \& Hummer 1965; Hummel \& Dachs 1992; Hummel 1994). Since the source function increases with depth, the effective local absorption profile becomes double-peaked (i.e. non-Gaussian) and somewhat broader than in the purely thermal case,

$$
\Delta v_{\mathrm{nsb}} \approx 1.5 \Delta v_{\mathrm{th}}[\ln (\tau \sec i)]^{1 / 2}
$$

(Avrett \& Hummer 1965) or $\approx 2.3 \ldots 3.9 \Delta v_{\text {th }}$ for $\tau=$ $10 \ldots 10^{3}$ at $i=0^{\circ}$. At low inclination, it is of same magnitude as kinematical broadening and causes winebottletype flank inflections. At very low inclination, it may produce single-peak, or in rare cases triple-peak profiles ${ }^{1}$. Above $i \approx 60^{\circ}$, it becomes less important than shear broadening.

Another broadening effect, Thomson scattering (e.g., Poeckert \& Marlborough 1978), affects only the line wings since its typical width is given by the thermal width of electrons.

Finally, at very high inclination $\left(i>80^{\circ}\right)$, shell absorption of photospheric light by the disk becomes important which causes a deep central depression below the stellar continuum in both $\mathrm{H} \alpha$ and $\mathrm{Fe}$ II profiles.

It is obviously useful to introduce a second parameter for classification, optical depth $\tau$, since NSB broadening depends on $\tau$. Since we are not intending to actually measure $\tau$, we simply use the terms $\tau \gg 1$ for $\mathrm{H} \alpha$ lines, and $\tau \lesssim 1$ for Fe II lines here.

In Fig. 1 we have collected four sets of $\mathrm{H} \alpha / \mathrm{Fe}$ II profiles with typical shapes. The leftmost profile arises from a nearly face-on disk, with $\Delta v_{\text {nsb }} \approx \Delta v_{\text {kin }} \approx \Delta v_{\text {th }} \gg \Delta v_{\text {sh }}$. The $\mathrm{H} \alpha$ profile exhibits a single peak (caused by overlap of the two innermost peaks of the convolved kinematical and NSB broadening functions, resp.) plus strong winebottletype inflections (due to the two outermost peaks of the convolved functions). The Fe II profile reflects the pure kinematical profile which is known to be double-peaked for thin disks (Huang 1972; Smak 1981; Hanuschik 1988).

The next profile set is typical of low to intermediateinclination disks. Now $\Delta v_{\text {kin }}>\Delta v_{\text {nsb }} \gg \Delta v_{\text {th }}>\Delta v_{\text {sh }}$,

\footnotetext{
${ }^{1}$ The original interpretation of winebottle inflections as evidence for "two-component structure" (Hanuschik 1986, HKK88) is not maintained anymore.
} 


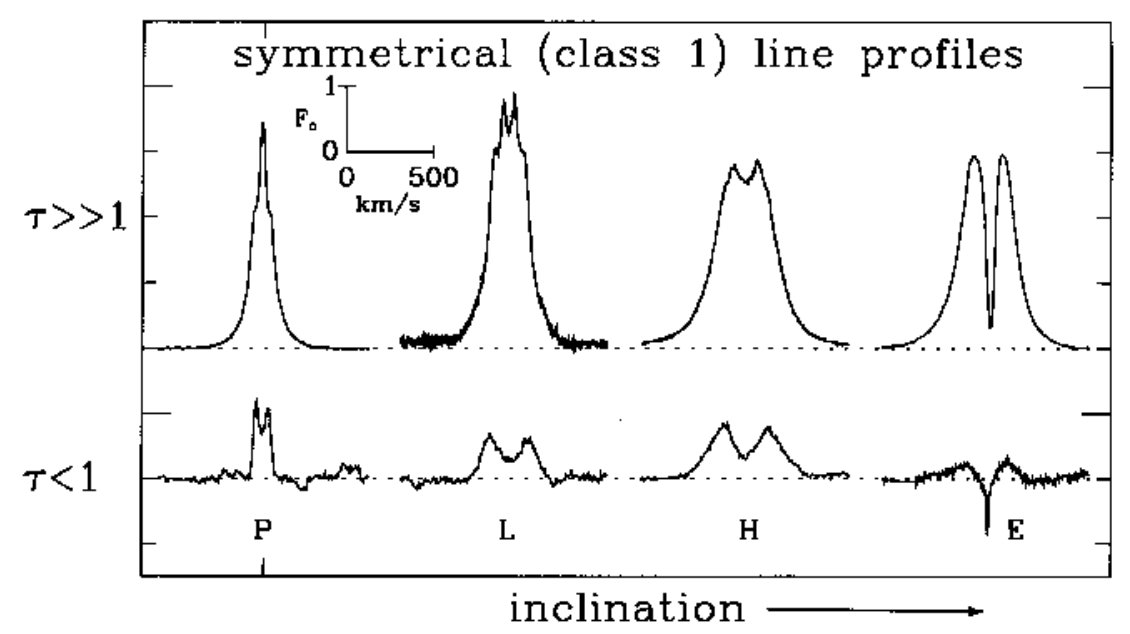

Fig. 1. Typical emission line shapes for symmetrical (class 1) profiles. We show four sets of H $\alpha$ (top) and Fe II (bottom) profiles, plotted on a radial velocity vs. flux scale and assorted according to the inclination indicators in Sect. 4.3. The Fe in profiles are expanded by a factor $k=10$, see Eq. (1). The four stars are, from left to right: HR 2825, measured in 1992 March $24=920324$ $\left(v \sin i \leq 40 \mathrm{~km} \mathrm{~s}^{-1}\right.$; full set of profiles in Fig. 34); 56 Eri $=$ HR 1508, $820816\left(180 \mathrm{~km} \mathrm{~s}^{-1}\right.$, Fig. 11); $\alpha$ Ara $=$ HR 6510,890926 $\left(250 \mathrm{~km} \mathrm{~s}^{-1}\right.$, Fig. 67); o Aqr $=$ HR 8402, 891001 (300 $\mathrm{km} \mathrm{s}^{-1}$, Fig. 75$)$

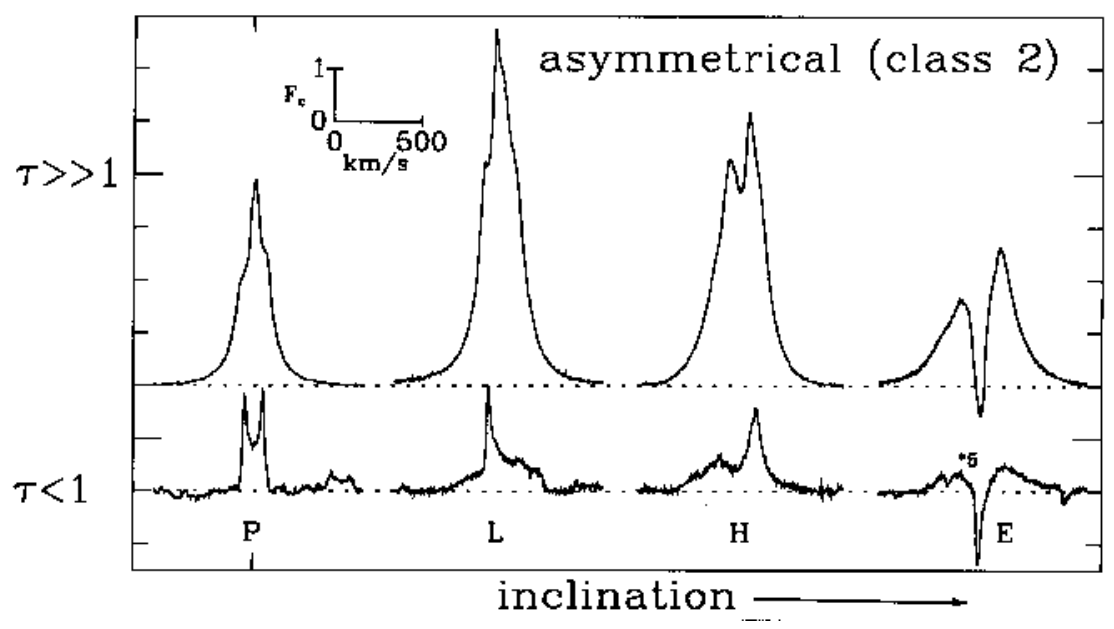

Fig. 2. Typical emission line shapes for asymmetrical (class 2) profiles. As in Fig. 1, we show four sets of H $\alpha$ and Fe in profiles; from left to right: HR 5223, $920324\left(v \sin i=70 \mathrm{~km} \mathrm{~s}^{-1}\right.$; full set of profiles in Fig. 56); $\delta$ Cen $=$ HR $4621,850228\left(220 \mathrm{~km} \mathrm{~s}{ }^{-1}\right.$, Fig. 50); Pleione $=$ HR 1180, 930909 (320 km s${ }^{-1}$, Fig. 9); 48 Lib = HR 5941 (400 km s${ }^{-1}$, Fig. 63)

and the two innermost peaks of the convolved kinematical and NSB profile are resolved.

The third profile set is representative of large inclinations, with $\Delta v_{\text {kin }} \gg \Delta v_{\text {sh }}>\Delta v_{\text {nsb }}>\Delta v_{\text {th }}$. NSB broadening is unimportant now (the winebottle shoulders have disappeared), while shear broadening deepens the central part of both $\mathrm{H} \alpha$ and Fe II profiles. Note especially the Vshape of the central depression in the Fe II profile.

Finally, the last set represents an edge-on disk $(i>$ $80^{\circ}$ ) where shear broadening and shell absorption act together to produce a deep central depression.

It is well known that the shape of the kinematical profile furthermore depends on the outer radius of the disk (peak separation) and on the emissivity law (slope of flanks). Since we are only interested here in a qualitative description of profile shapes, we neglect these influences. Fits to Fe II emission lines may be found in Hanuschik (1988).

\subsection{Kinematics}

Up to now we have only treated symmetric line profiles. Implicitely we have thus restricted ourselves to disks with an axisymmetrical velocity field and density distribution, which most likely corresponds to a quasi-Keplerian disk in hydrostatic equilibrium. HKK88 have coined the term 


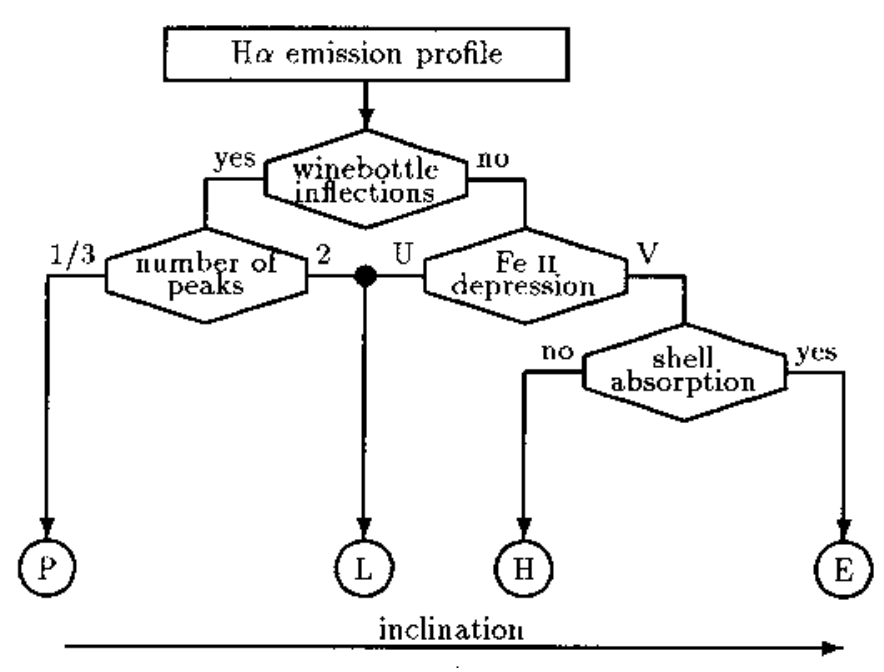

Fig. 3. Classification scheme for Be emission line shapes

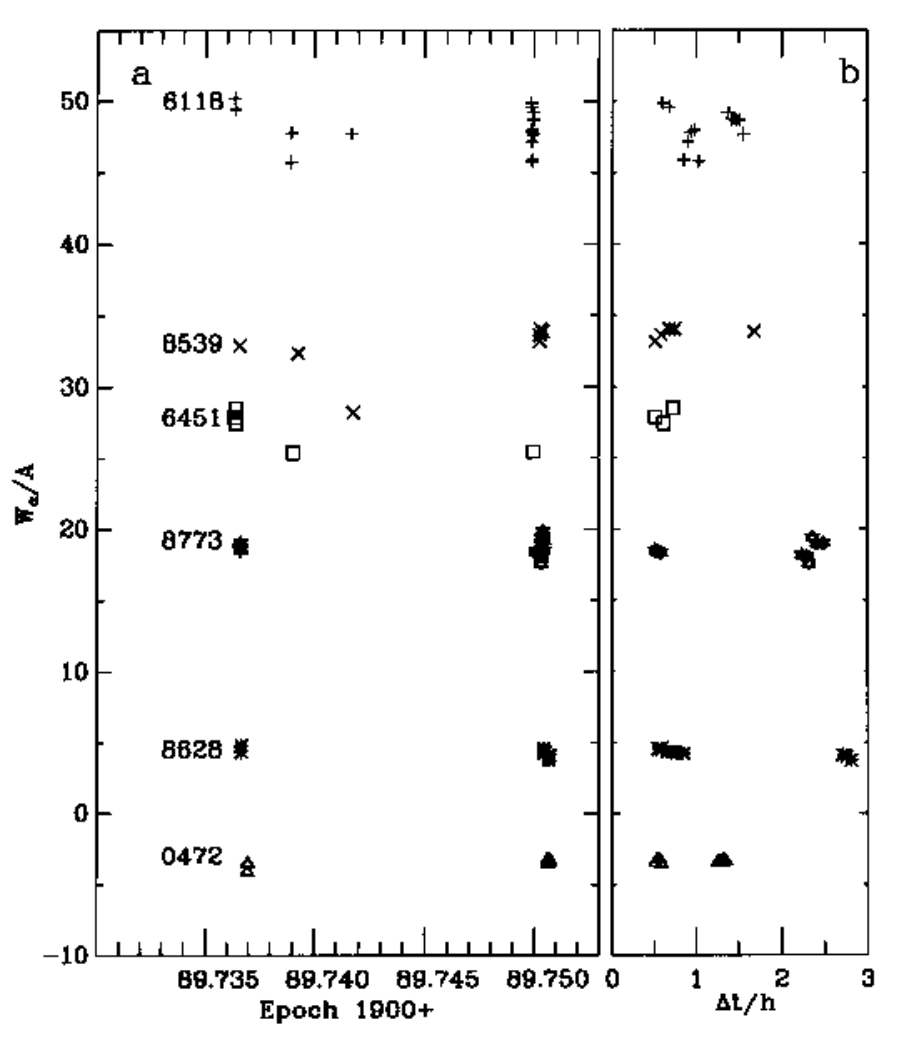

Fig. 4. Investigation of short-term variability. We have plotted here $W_{\alpha}$ for 6 programme stars which have been frequently measured between 1989 Sep. $26(=1989.736-7)$ and Oct. 1 (= 1989.750-1). The left panel shows all data of this epoch, the right panel a blow-up of the data for Oct. 1 (for HR 6451: Sep. 26 ), sorted on a timescale $\Delta t$ measured in hours, with arbitrary zero-point class 1 for this type of profiles. A second major class exists which collects all profiles which are asymmetric in a certain typical sense: they are cyclically variable, and they exhibit, in case of Fe II lines, a typical "steeple-like" shape in phases of strong asymmetry. Hanuschik et al. (1995a) interpret these class 2 profiles as being due to a distorted Keplerian disk hosting a global density wave.

Figure 2 shows a selection of line profiles typical of class 2 shapes. For pole-on disks, we have found only mildly asymmetric examples in our atlas. For $i \approx 30^{\circ}$, the typical steeple-type shape for the kinematical profile is visible in the Fe II line, with a very steep and narrow dominating peak, and an almost suppressed secondary peak. Around the corresponding radial velocity position in $\mathrm{H} \alpha$, a strong flank inflection and a single peak are visible, both caused by the convolution of the NSB function and the dominating kinematical peak. On the red side of $\mathrm{H} \alpha$, no pronounced structure is visible, corresponding to the fact that the secondary kinematical peak is suppressed.

At high inclinations, the flank inflections disappear like in class 1 profiles. Finally shell absorption arises in edgeon disks, with the asymmetry of the velocity field reflected in the asymmetry of the absorption trough.

\subsection{Classification}

We have classified the profile shapes observed in our programme stars with the scheme proposed in the previous section. Since essentially all $\mathrm{H} \alpha$ profiles in our atlas are optically thick (winebottle structure becomes visible if $F_{\alpha} / F_{\text {c }} \gtrsim 1.5$, see HR 5193 in Fig. 55), and Fe II profiles are optically thin (they never show winebottle inflections), only two dimensions remain for each observed $\mathrm{H} \alpha$ or Fe II profile. These are (inclination parameter $) \times($ kinematical parameter).

The inclination indicator is coded as (see Fig. 3)

- $P$ for profiles from pole-on disks (single [merged] NSB peak and winebottle-type shoulders which may form together triple peaks in extreme cases),

$-L$ for low to intermediate inclinations (double NSB/kinematical peaks plus weaker shoulders),

$-H$ for high inclinations (double kinematical peaks, no shoulders, V-shaped central depression in Fe II lines),

- $E$ for edge-on disks (deep central depression, below continuum in Fe II lines).

An example for triple peaks is provided by $\chi$ Oph (Fig. 62). In one single case, a P-type profile is optically thick enough to show $N S B$ peak splitting, i.e. $\Delta v_{\text {nsb }}>\Delta v_{\text {kin }}$ (HR 2825 in 1985, see Fig. 34). We classify the profiles merely from their shape, the stellar $v \sin i$ value does not enter. We therefore provide an independent (though not very precise) measure for inclination here. The estimated inclination angles are $i \lesssim 10^{\circ}$ for P-type profiles, $10^{\circ} \lesssim$ $i \lesssim 60^{\circ}$ for L-type profiles, $60^{\circ} \lesssim i \lesssim 80^{\circ}$ for type-H, and $i \gtrsim 80^{\circ}$ for type-E. 
Table 4. Short-term variations (Sep. 26-Oct. 1, 1989)

\begin{tabular}{rrrlclccc}
\hline Star & \multicolumn{3}{c}{$\Delta t \leq 3$ hours (Oct. 1) } & & \multicolumn{3}{c}{$\Delta t=5$ days (Sep.26-Oct.1) } \\
\cline { 2 - 3 } HR & $N$ & mean $W_{\alpha} / \AA$ & $\Delta W_{\alpha} / \AA$ & $\Delta W_{\alpha} / W_{\alpha}$ & & mean $W_{\alpha} / \AA$ & $\Delta W_{\alpha} / \AA$ & $\Delta W_{\alpha} / W_{\alpha}$ \\
\hline 472 & 9 & -3.40 & 0.13 & $4 \%$ & & -3.60 & 0.20 & $6 \%$ \\
6118 & 12 & 48.1 & 1.9 & $4 \%$ & & 48.1 & 2.0 & $4 \%$ \\
6451 & $3^{a}$ & 27.9 & 0.5 & $2 \%$ & & 26.2 & 1.2 & $5 \%$ \\
8539 & 5 & 33.8 & 0.3 & $1 \%$ & & 31.8 & 1.4 & $4 \%$ \\
8628 & 9 & 4.26 & 0.27 & $6 \%$ & & 4.44 & 0.25 & $6 \%$ \\
8773 & 13 & 18.7 & 0.7 & $4 \%$ & & 18.7 & 0.05 & $<1 \%$ \\
\hline
\end{tabular}

${ }^{a}$ Sep. 26, 1989

The kinematical state is indicated by " 1 " for class 1 profiles, and by "2" for class 2 profiles.

The last column of Table 2 contains the shape coding. Uncertain coding is indicated by a colon, ... denotes profiles which do not fit in our system (due to short-term variability or irregular profile shape), and "abs" indicates a pure absorption line. Symbols in brackets denote weak $\mathrm{H} \alpha$ emission lines which do not show flank inflections due to low optical depth.

\section{Short-term variability of emission lines}

\subsection{Days or shorter}

It is well known that Be stars may show at times line profile variability on short timescales (e.g., Harmanec 1991). Most such observations concern photospheric absorption lines where low-amplitude bumps have been found, moving across the line profile and interpreted as evidence for pulsations. The observations of Smith (1989) of faint emission bumps in $\lambda$ Eri are another type of short-term phenomenon, indicating photosphere-disk interaction.

Usually we have covered in our atlas typical timescales of months up to years. In September/October 1989, we have furthermore systematically searched for short-term $\mathrm{H} \alpha$ variability in six programme stars: $\mathrm{HR} 472$, HR 6118 , HR 6451, HR 8539, HR 8628, and HR 8773. We have covered timescales $\Delta t$ of five days, one day, a few hours, and a few minutes.

We have measured the equivalent width of $\mathrm{H} \alpha, W_{\alpha}$, by interpolation of the local stellar continuum across the line profile. The results of this search are collected in Table 4 and plotted in Fig. 4.

$-\Delta t \lesssim$ hours: All these six stars show scattering in $W_{\alpha}$ on a relative scale $\leq 6 \%$, typically $4 \%$. As a careful check shows, no variability in line profile shape (finestructure) is found. The scatter affects the whole line profile.

$-\Delta t \sim$ days: We find the same scattering behaviour with a typical value of $4 \%$. Again no finestructure variability is found.
It follows that the scatter found on both timescales is not physical, but entirely or mostly due to reduction and measuring uncertainties caused by insecure definition of the interpolated stellar continuum. This conclusion is supported by the facts that the scatter found i) is the same on timescales of minutes, hours and days, ii) is not accompanied by finestructure variability, iii) is the same for $\mathrm{H} \alpha$ absorption lines (in $\alpha$ Eri), faint $\mathrm{H} \alpha$ emission ( $\epsilon$ PsA), or strong emission.

We conclude that we have found no indication of shortterm variability in emission lines on timescales of one day or shorter. Contentions sometimes found in the literature (e.g. Ghosh 1988; Hubert et al. 1988) are not supported by data in our atlas. This is at least true for diskinduced variability (i.e. variability of emission strength) which should not be confused with photosphere-induced variability (variations of the underlying stellar absorption line).

\subsection{Few days}

The shortest timescale of emission line variability found here is established by two different types of finestructure variability, "binary-type" and "flickering emission-type".

Binary-type. In the stars $\zeta$ Tau, HR 2142, and $\pi$ Aqr, timescales of $<24^{\mathrm{d}}$ ( $\zeta$ Tau), few days (HR 2142) and $<12^{\mathrm{d}}(\pi$ Aqr) have been found (see Figs. $17 \mathrm{~d}, 22 \mathrm{~d}, 78 \mathrm{~d}$ ). Two of these stars are known as binaries $\left(P=132.91^{\mathrm{d}}\right.$ and $80.86^{\mathrm{d}}$ for $\zeta$ Tau and HR 2142, resp., Pols et al. 1991). In case of HR 2142, the emission line shape furthermore varies with the orbital period (Fig. 22d). This demonstrates that the Be disk is at least distorted by the companion star. In case of $\pi$ Aqr, no evidence for duplicity is known at present. Its rapid line profile variability, however, could be an indicator for binarity.

Flickering emission. For stars showing flickering emission, timescales of a day are well established and identified with the dynamical (= orbital) period in the disk close to the stellar surface. The density distribution in these disks is still inhomogeneous if they are observed in the process of formation. After a few such timescales, cir- 
cularization of orbits is achieved and line profile shapes remain constant (with, however, still varying equivalent width). From our atlas, the stars $\alpha$ Eri, $\lambda$ Eri, HR 3498 (?), and, as prototype, $\mu$ Cen (Hanuschik et al. 1993) belong to this subclass.

Acknowledgements. We would like to thank Prof. J. Dachs for many stimulating discussions about Be stars in general, and Prof. A. Slettebak for making available his Balmer line profiles from 1989 in electronic form.

\section{References}

Andrillat Y., 1983, A\&AS 53, 319

Andrillat Y., Fehrenbach C., 1982, A\&AS 48, 93

Andrillat Y., Jaschek M., Jaschek C., 1988, A\&AS 72, 129

Andrillat Y., Jaschek M., Jaschek C., 1990, A\&AS 84, 11

Andrillat Y., Jaschek M., Jaschek C., 1994, A\&AS 103, 135

Avrett E.H., Hummer D.G., 1965, MNRAS 130, 295

Baade D., 1987, in: Slettebak A., Snow T.P. (eds.), Physics of Be stars, IAU Coll. 92. Cambridge Univ. Press, p. 361

Baade D., Dachs J., van de Weygaert R., Steeman F., 1988, A\&A 198, 211

Ballereau D., Alvarez M., Chauville J., Michel R., 1987, Rev. Mex. A\&A 15, 29

Barker P., 1986, PASP 98, 44

Dachs J., Eichendorf W., Schleicher H., Schmidt-Kaler Th., Stift M., Tüg H., 1981, A\&AS 43, 427

Dachs J., Hanuschik R.W., Kaiser D., et al., 1986, A\&AS 63, 87

Dachs J., Hummel W., Hanuschik R.W., 1992, A\&AS 95, 437

Doazan V., Sedmak G., Barylak M., Rusconi L., 1991, A Be Star Atlas, esa SP-1147

Ghosh K.K., 1988, Vistas Astron. 31, 281

Ghosh K.K., Kuppuswamy K., Pukalenthi S., Selvakumar G., 1991, AJ 102, 1191

Gies D.R., Willis C.Y., Penny L.R., 1993, PASP 105, 281

Guo Yulian, 1994, Inf. Bull. Var. Stars, No. 4112
Hanuschik R.W., 1986, A\&A 166, 185

Hanuschik R.W., 1987, A\&A 173, 299

Hanuschik R.W., 1988, A\&A 190, 187

Hanuschik R.W., Kozok J., Kaiser D., 1988, A\&A 189, 147 $(=$ HKK 88$)$

Hanuschik R.W., Dachs J., Baudzus M., Thimm G., 1993, A\&A 274, 356

Hanuschik R.W., Hummel W., Dietle O., Sutorius E., 1995a, A\&A 300, 163

Hanuschik R.W., Hummel W., Štefl S., Vrancken M., 1995b, Inf. Bull. Var. Stars No. 4174

Harmanec P., 1991, in: Baade D. (ed.), ESO Workshop on Rapid Variability of OB stars: Nature and Diagnostic Value. ESO Garching, p. 265

Horne K., Marsh T., 1986, MNRAS 218, 761

Huang S.-S., 1972, ApJ 171, 549

Hubert A.M., Hubert H., Dagostinoz B., Floquet M., 1988, in: Cayrel de Strobel G., Spite M. (eds.), Impact of very high S/N spectroscopy on stellar physics, IAU Symp. 132. Kluwer, p. 131

Hummel W., Dachs J., 1992, A\&A 262, L17

Hummel W., 1994, A\&A 289, 458

Jarad M.M., Hilditch R.W., Skillen I., 1989, MNRAS 238, 1085

Peters G., 1979, ApJS 39, 175

Peters G., 1983, PASP 95, 311

Peters G., 1986, ApJ 301, L61

Peters G., 1988, PASP 100, 207

Poeckert R., Marlborough M., 1978, ApJ 220, 940

Pols O.R., Coté J., Waters L.B., Heise J., 1991, A\&A 241, 419

Porri A., Stalio R., 1988, A\&AS 75, 371

Slettebak A., 1982, ApJS 50, 55

Slettebak A., Collins G.W., Truax R., 1992, ApJS 81, 335 $(=\mathrm{S} 192)$

Smak J., 1981, Acta Astron. 31, 395

Smith M., 1989, ApJS 71, 357

Štefl S., 1994, IAU Circ. 6098

Struve O., 1931, ApJ 73, 94 

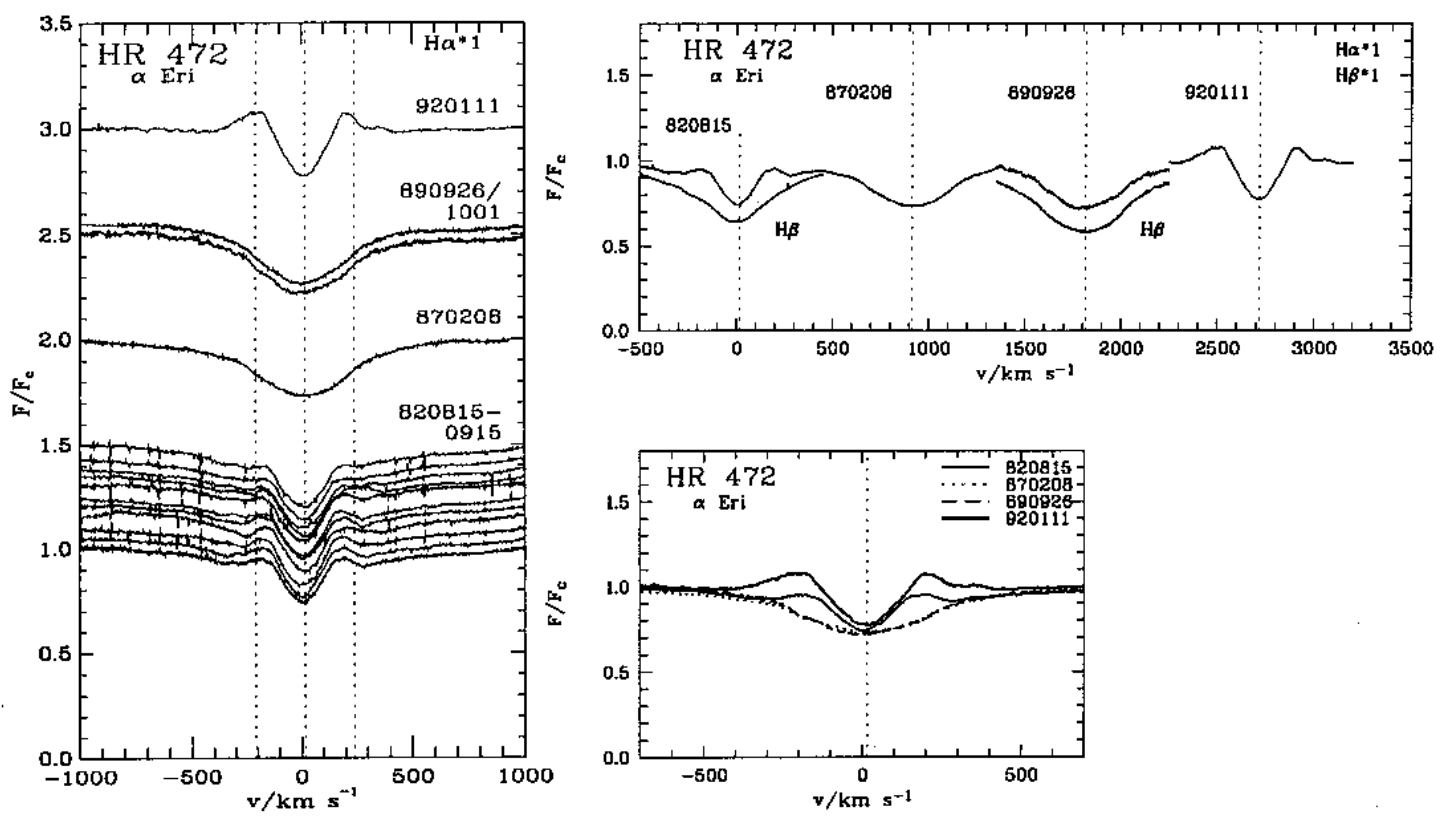

Fig. 5. High-resolution $\mathrm{H} \alpha$ and $\mathrm{H} \beta$ profiles for $\mathrm{HR} 472=\alpha$ Eri. All profiles are on a heliocentric radial velocity scale. The peculiar velocity $v_{\text {pec }}$ of the star, as taken from the Bright Star Catalogue, is plotted as dotted central vertical line in a), and dotted lines in $\mathbf{b}$ ) and $\mathbf{c}$ ). The other two vertical lines in a) mark $v_{\text {pec }} \pm v \sin i$. a) shows a vertical time sequence; profiles are shifted by arbitrary amounts, where - if possible - an attempt has been made to indicate the time shift by corresponding vertical shifts. Dates are given in the format 820815 = August 15, 1982. Profiles from 1982 (bottom to top): 0815, 16, 17, 18, $19,20,29,30,0913,14,15$. b) usually shows the profile set from a) in a horizontal display (here: only a subset). The radial velocity shift is indicated by the distance between two dotted lines. c) compares all $\mathrm{H} \alpha$ profiles plotted on top of each other
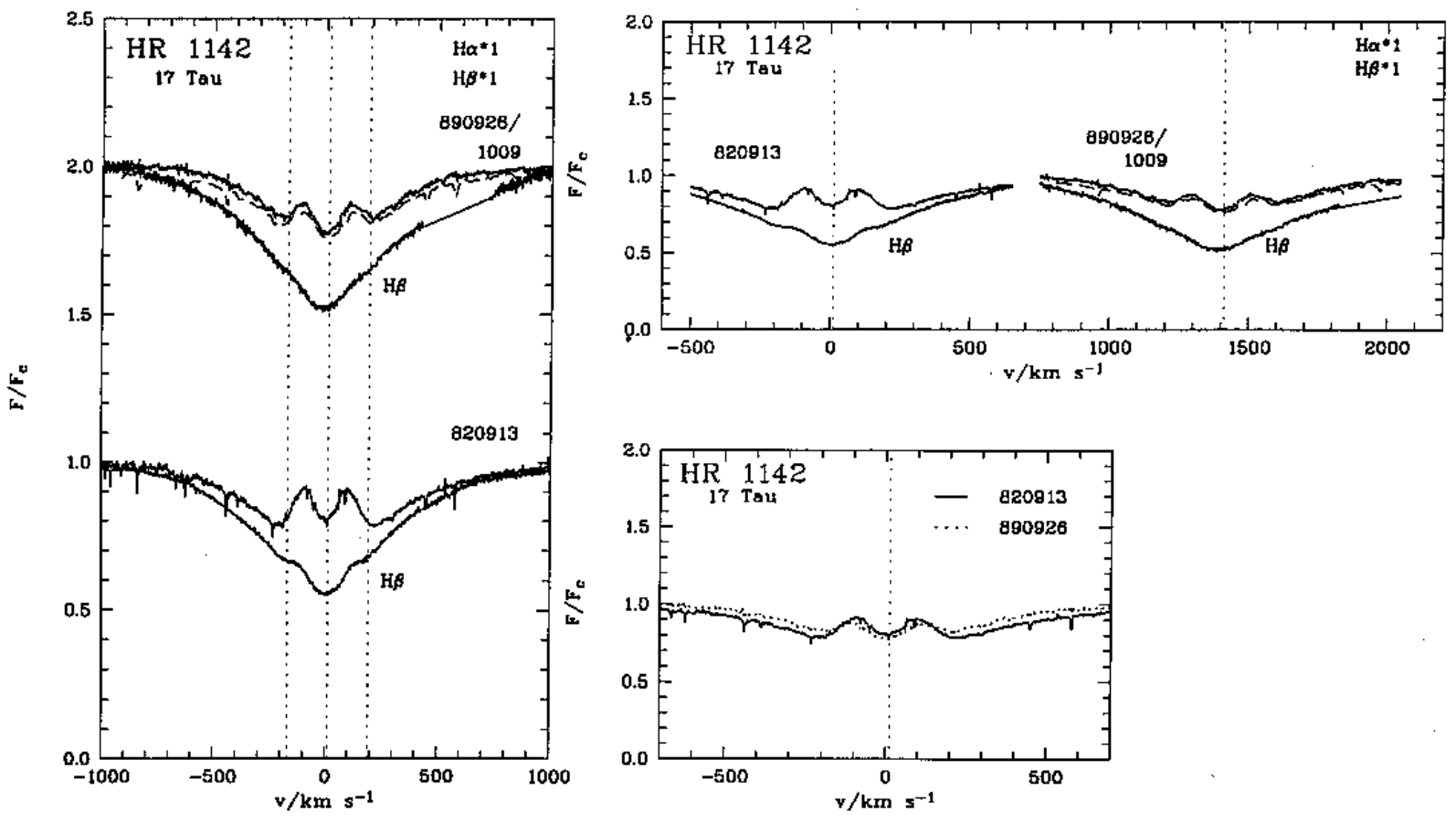

Fig. 6. Same as Fig. 5, for HR $1142=17$ Tau. Here, and in all following plots, the convention is that profiles plotted as broken line have been measured on the date given behind the slash (here: 891009 in a)) 

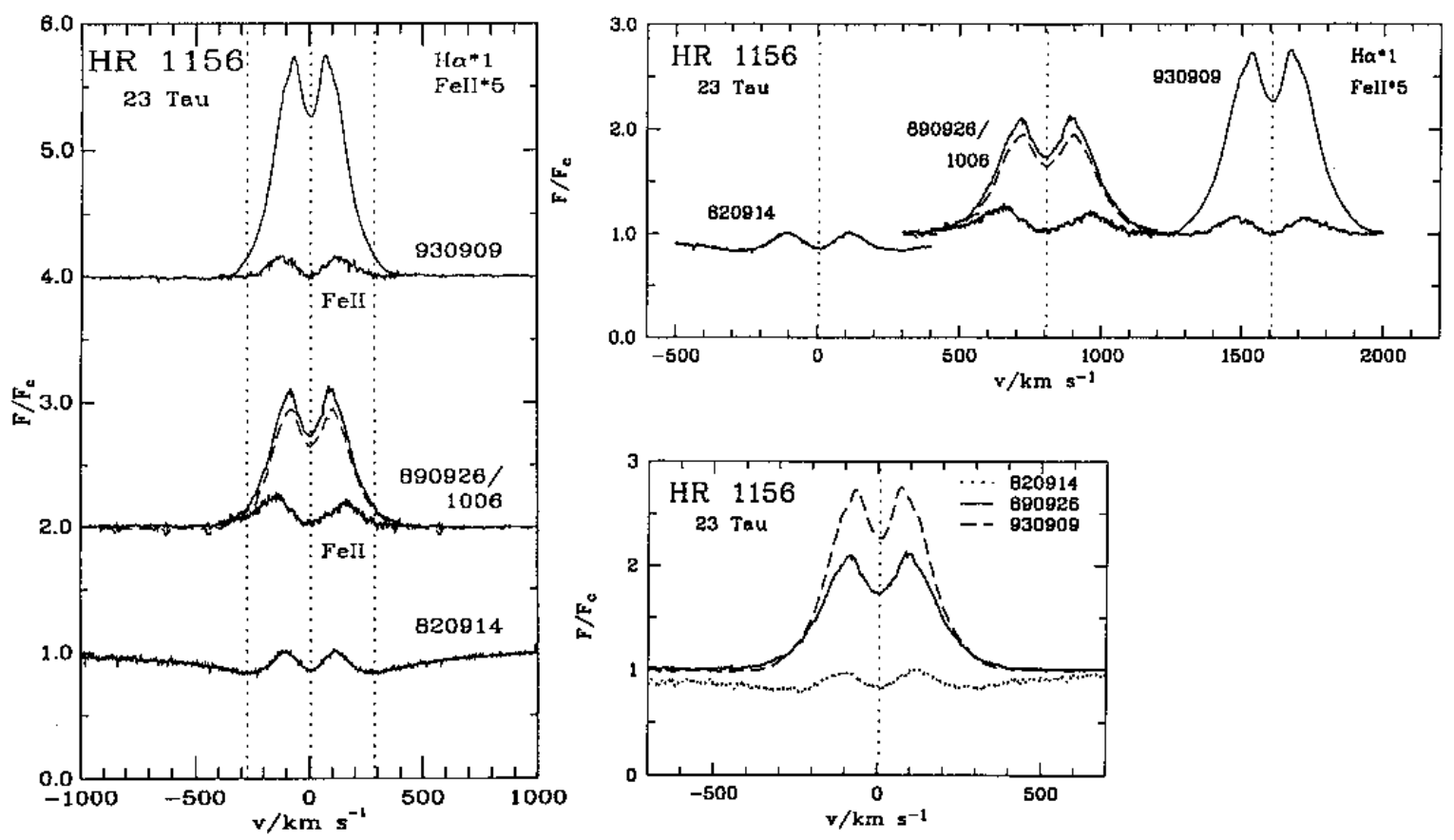

Fig. 7. As Figs. 5 and 6, for HR $1156=23$ Tau. Here, and in most other following plots, we show in a) and b) a comparison of quasi-simultaneously measured $\mathrm{H} \alpha$ and Fe II lines. If not otherwise noted, we have plotted the Fe II $\lambda 5317$ line. The scaling factor for the flux enhancement (Eq. 1) is given in the figure
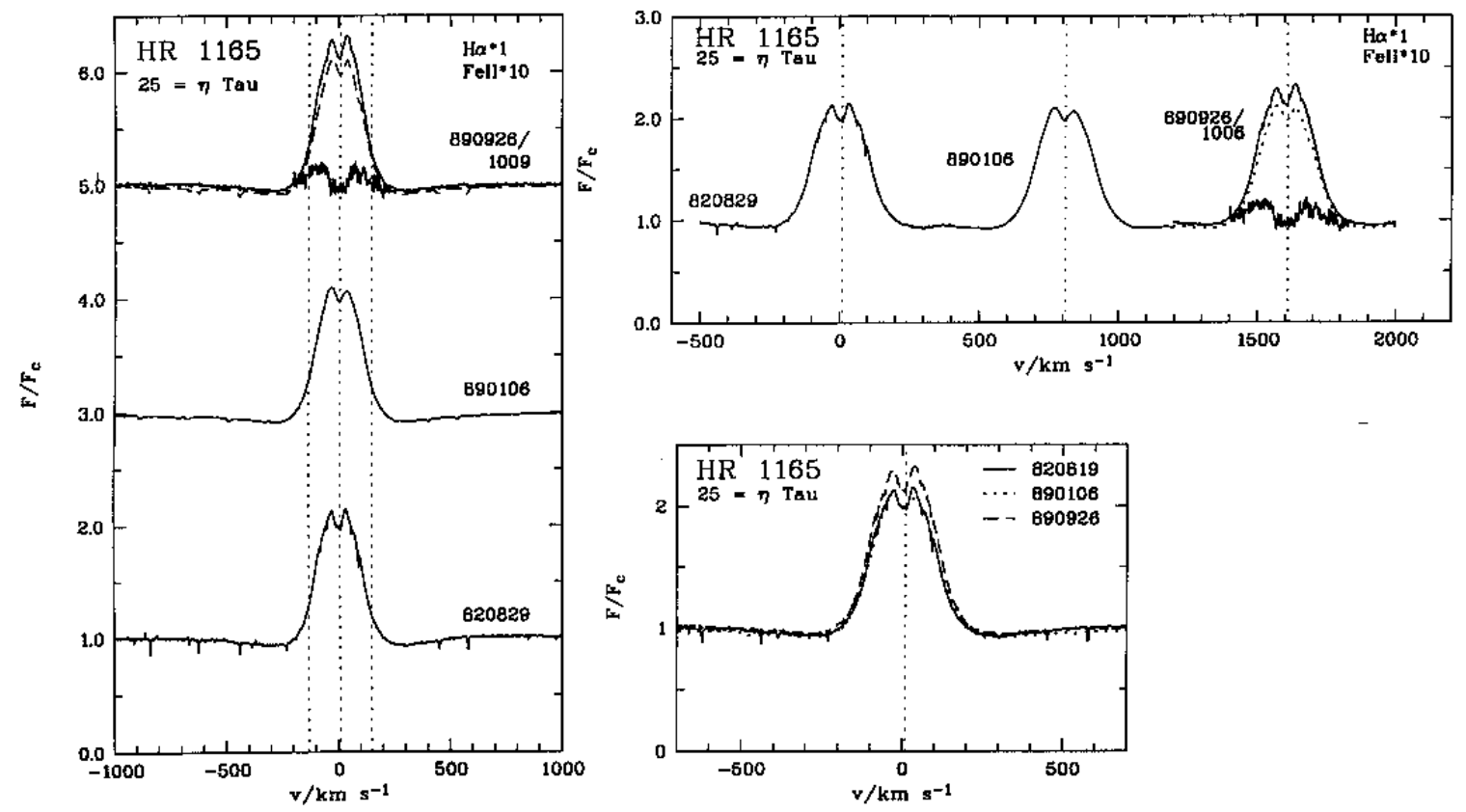

Fig. 8. $\mathrm{H} \alpha$ and Fe ir profiles for HR 1165 

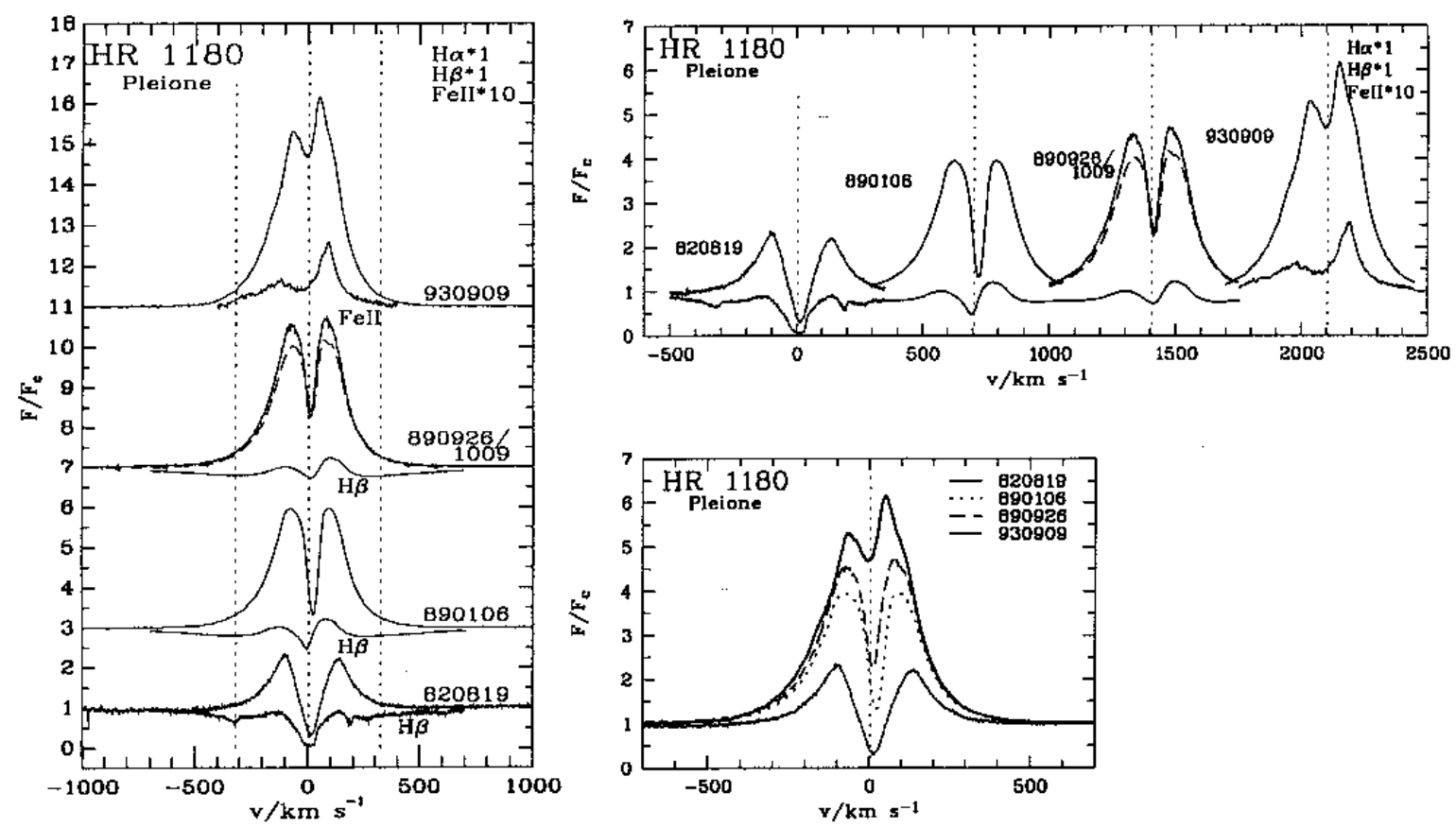

Fig. 9. $\mathrm{H} \alpha, \mathrm{H} \beta$ and $\mathrm{Fe}$ in profiles for HR 1180. Note the transition from Be-shell spectrum (until 1989) to Be spectrum (1993)
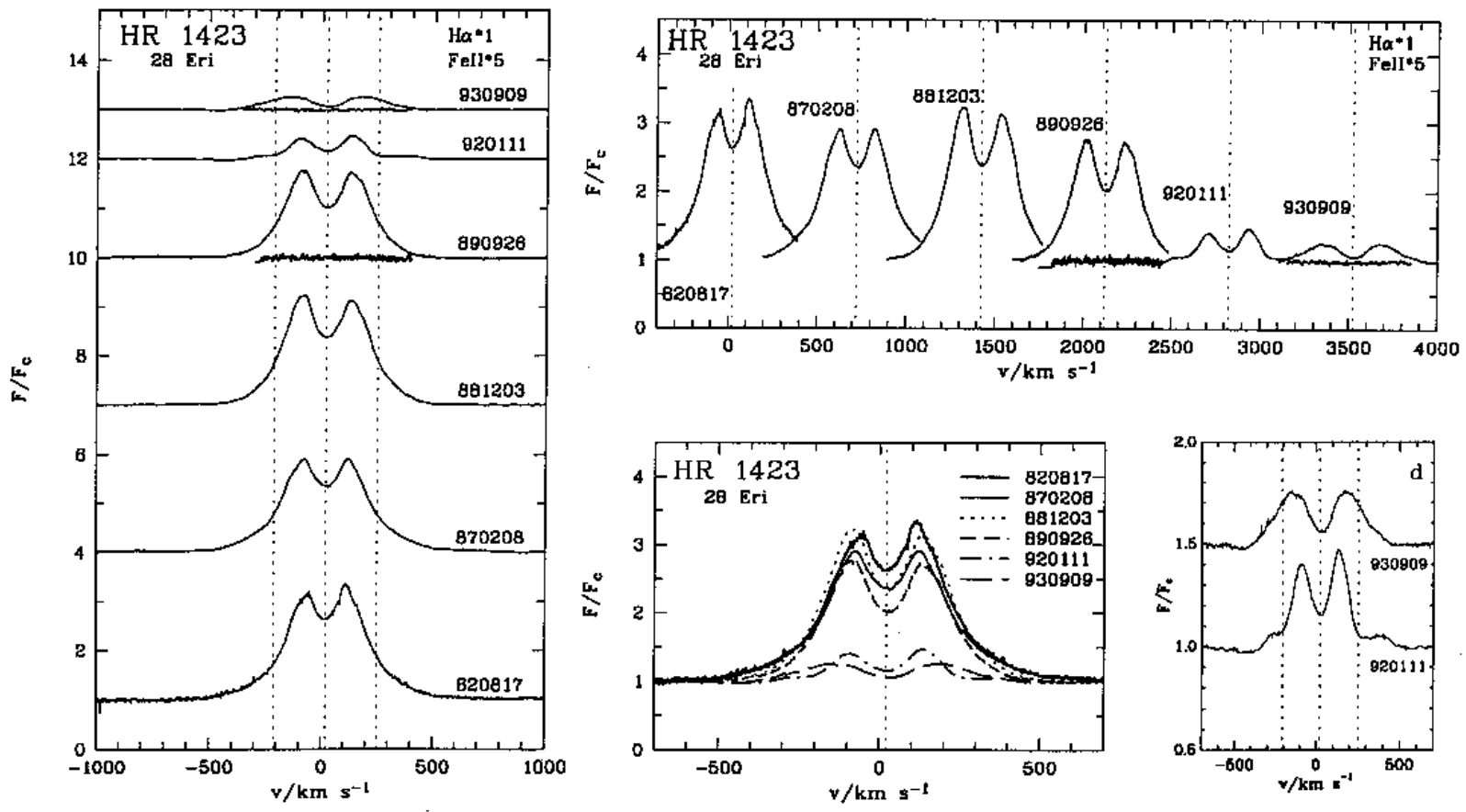

Fig. 10. H $\alpha$ profiles and Fe II measurements for HR 1423. A close-up of the unusual H $\alpha$ profile from 1992 is shown in panel d 

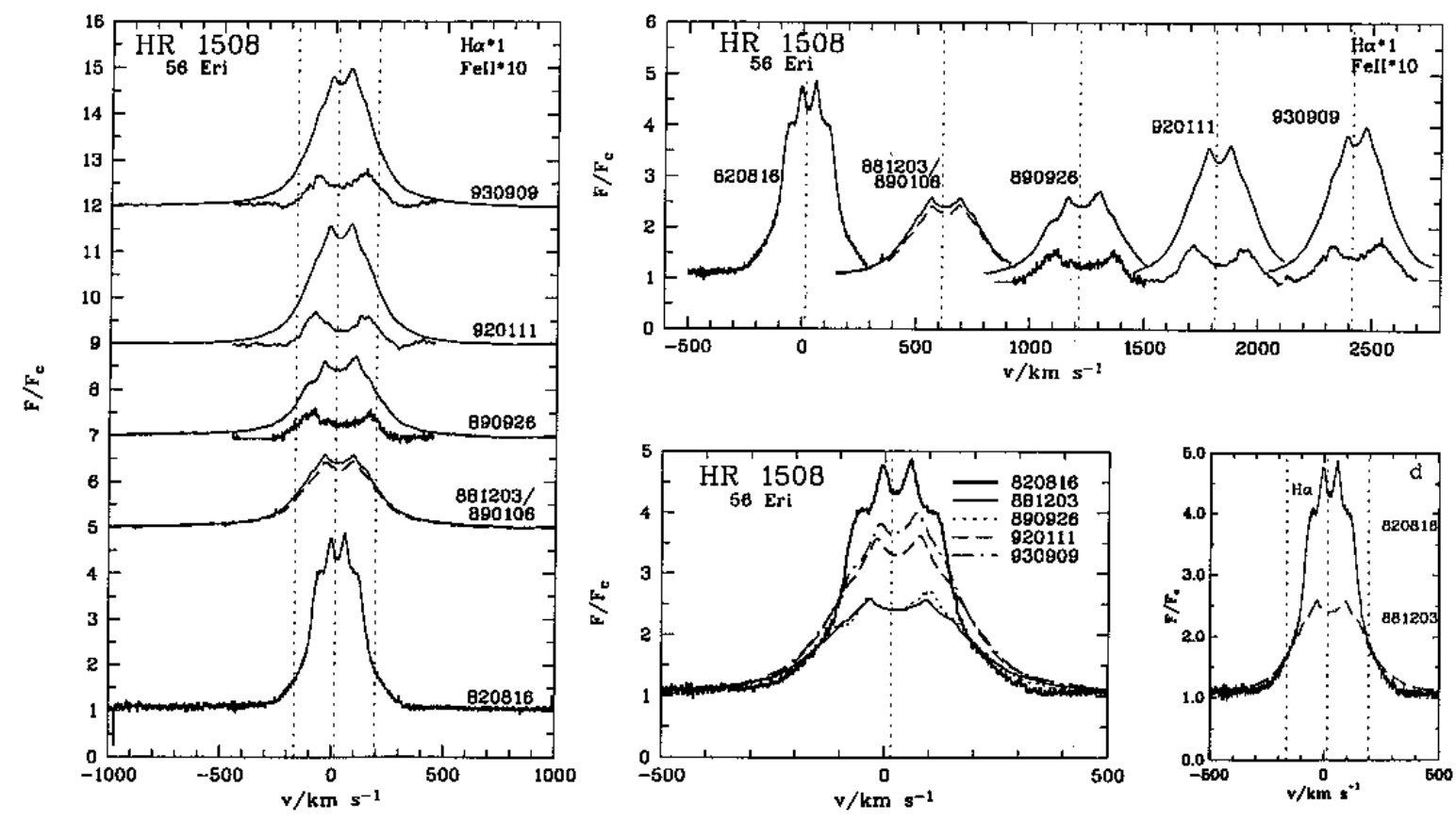

Fig. 11. H $\alpha$ and Fe in profiles for HR 1508. Panel d shows the change of line width in H $\alpha$ between 1982 and 1988
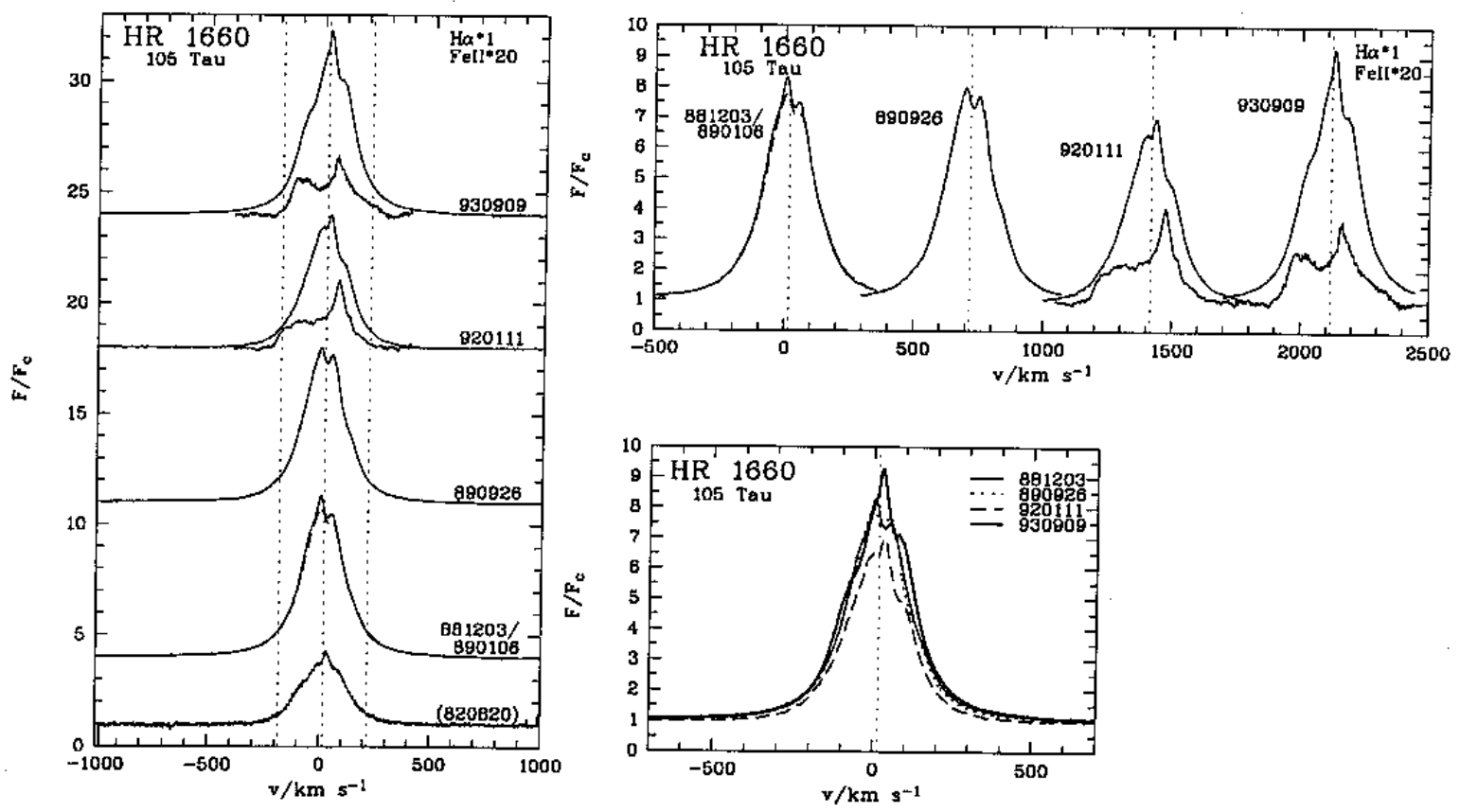

Fig. 12. H $\alpha$ and Fe in profiles for HR 1660. The 820820 profile has an unknown flux scale, only the profile shape is usable for interpretation 


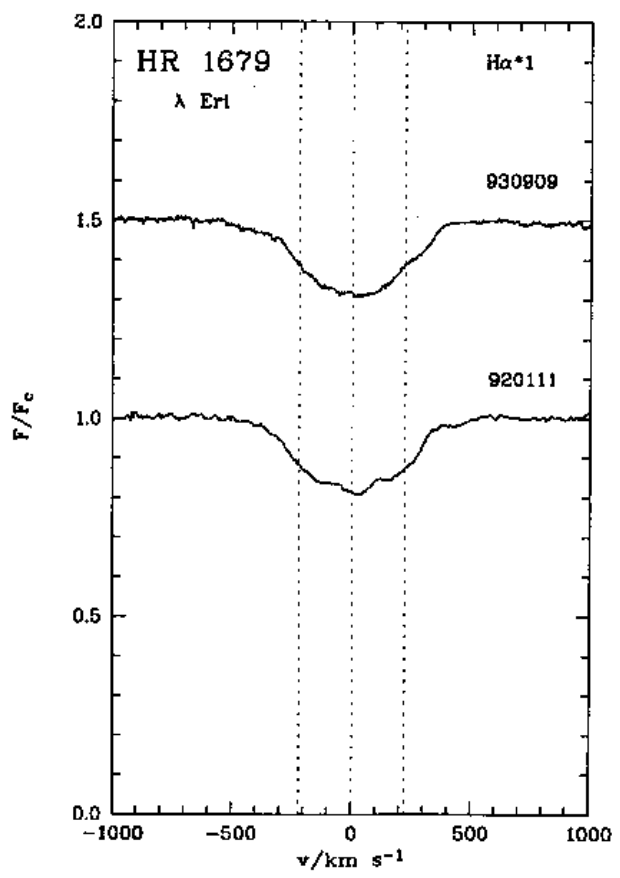

Fig. 13. $\mathrm{H} \alpha$ profiles for $\mathrm{HR} 1679$
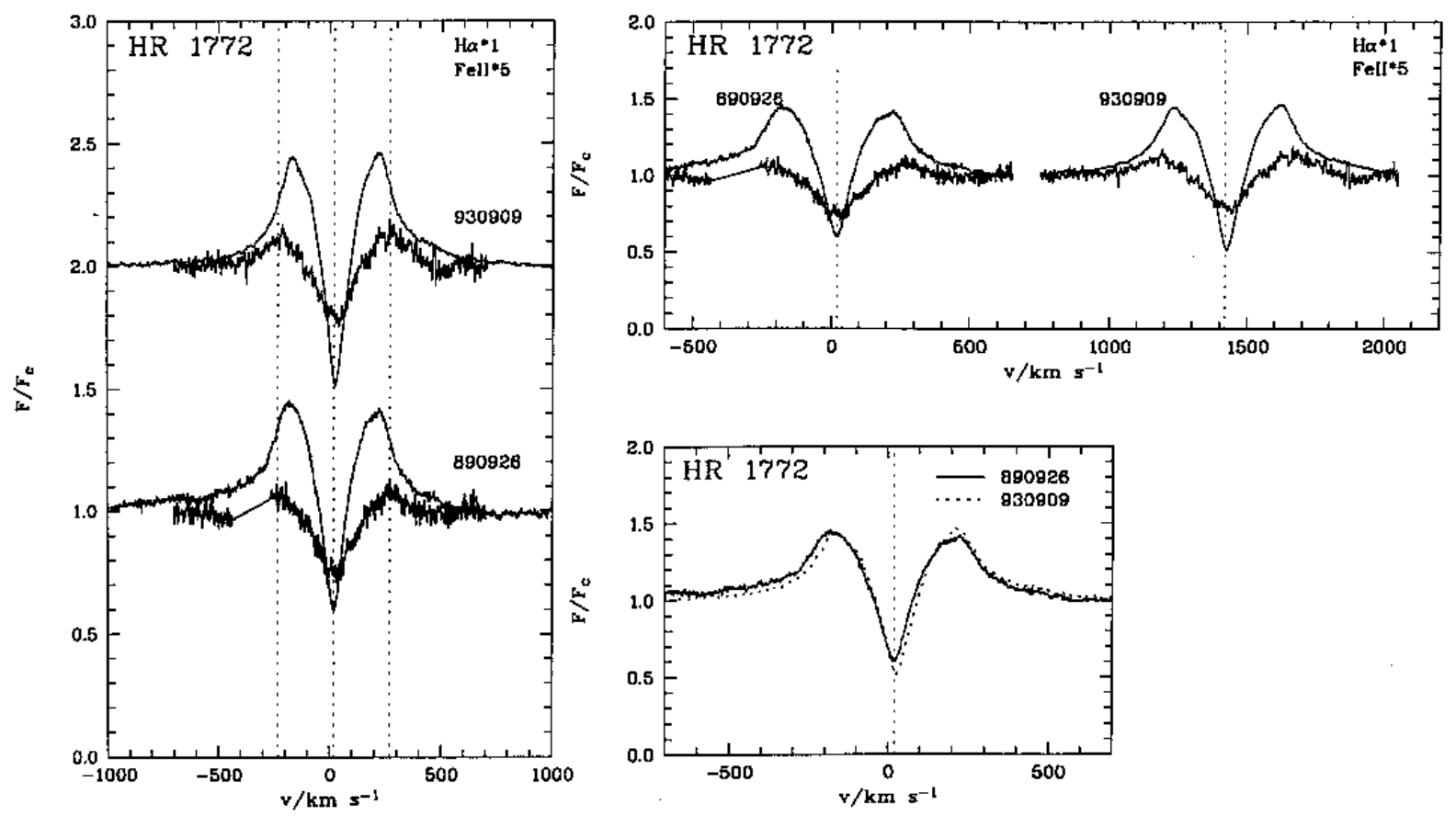

Fig. 14. $\mathrm{H} \alpha$ and $\mathrm{Fe}$ II profiles for $\mathrm{HR} 1772$ 

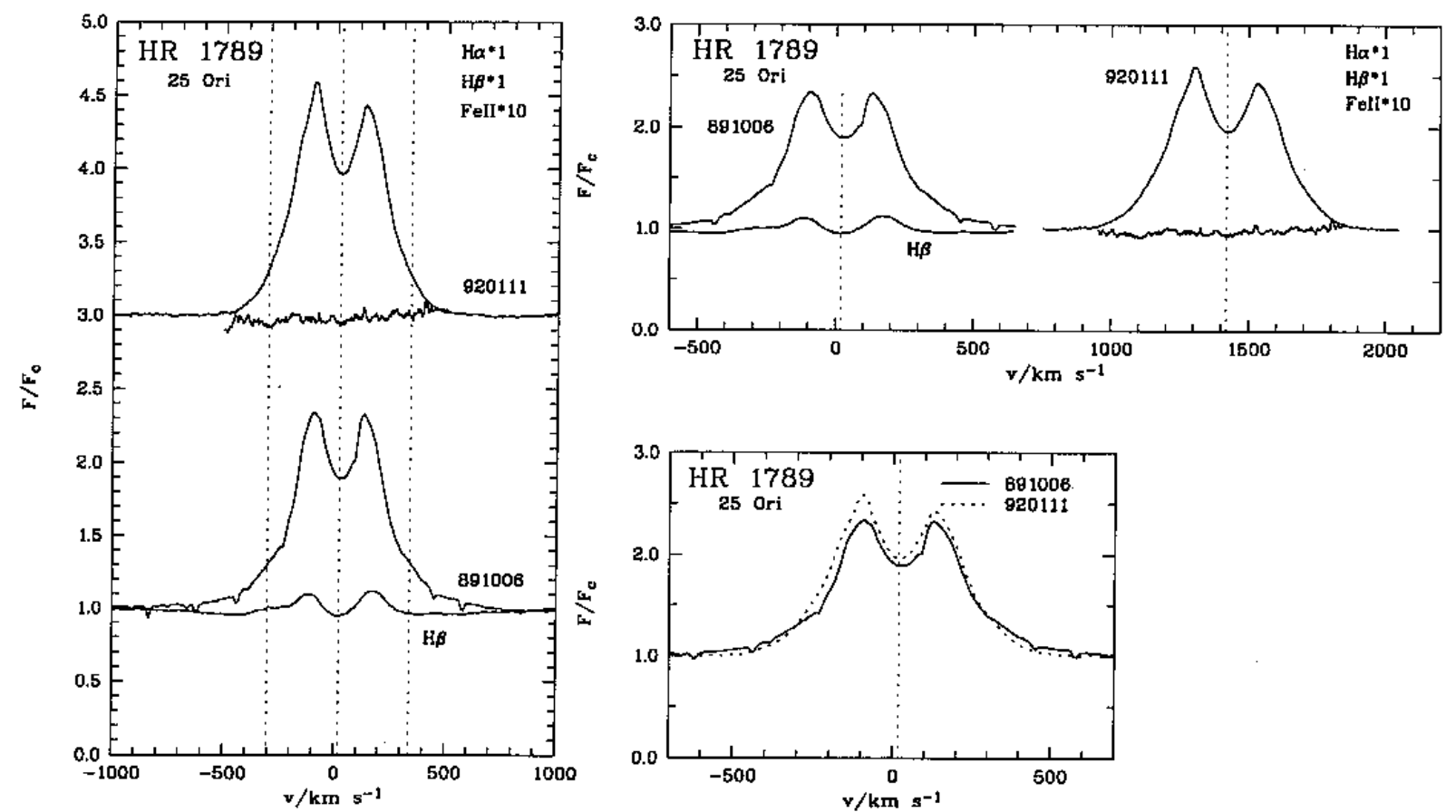

Fig. 15. $\mathrm{H} \alpha, \mathrm{H} \beta$ and $\lambda 5317$ profiles for $\mathrm{HR} 1789$ (no emission visible in Fe II)

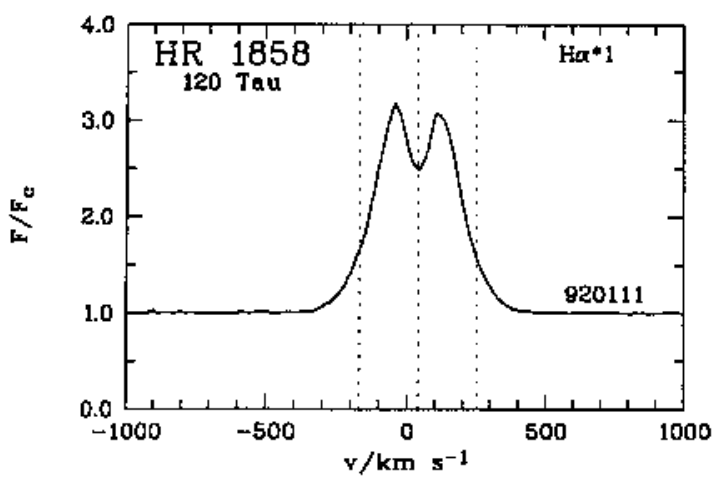

Fig. 16. $\mathrm{H} \alpha$ profile for $\mathrm{HR} 1858$ 

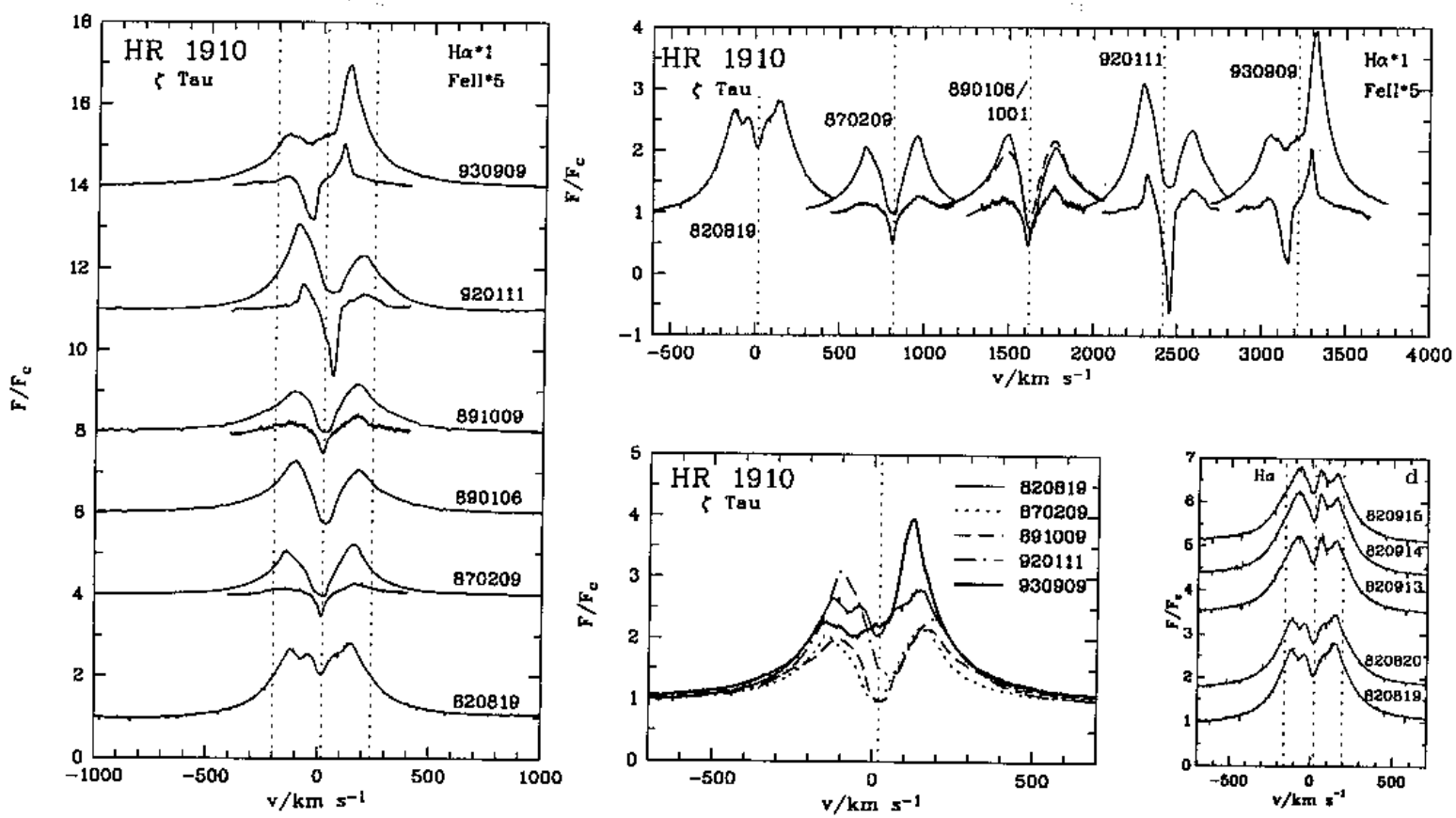

Fig. 17. H $\alpha$ and Fe in profiles for HR 1910. Note that by the expansion of the Fe in profiles also the shell troughs are affected
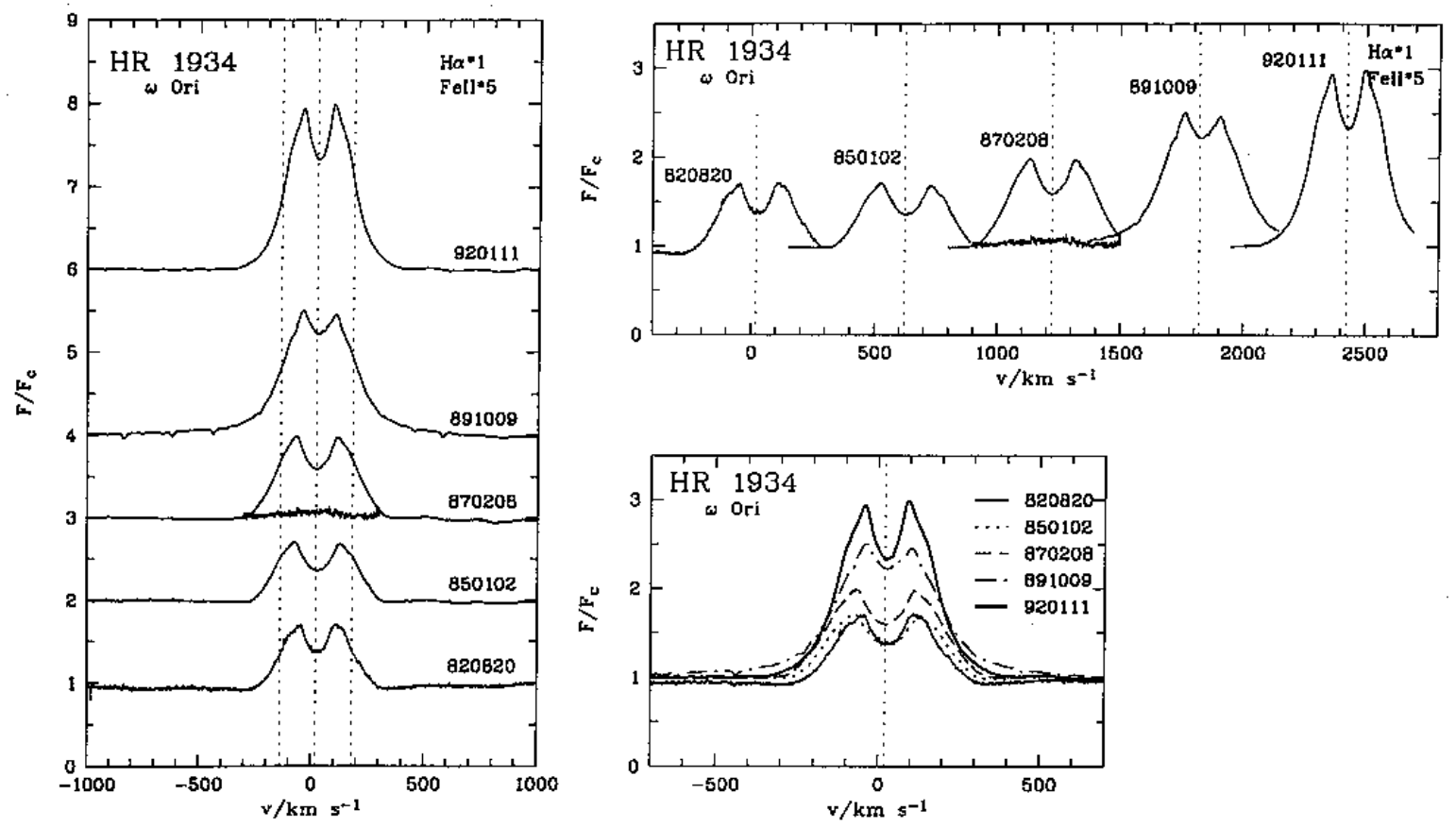

Fig. 18. H $\alpha$ and $\lambda 5317$ profiles for HR 1934 (no emission visible in Fe II) 

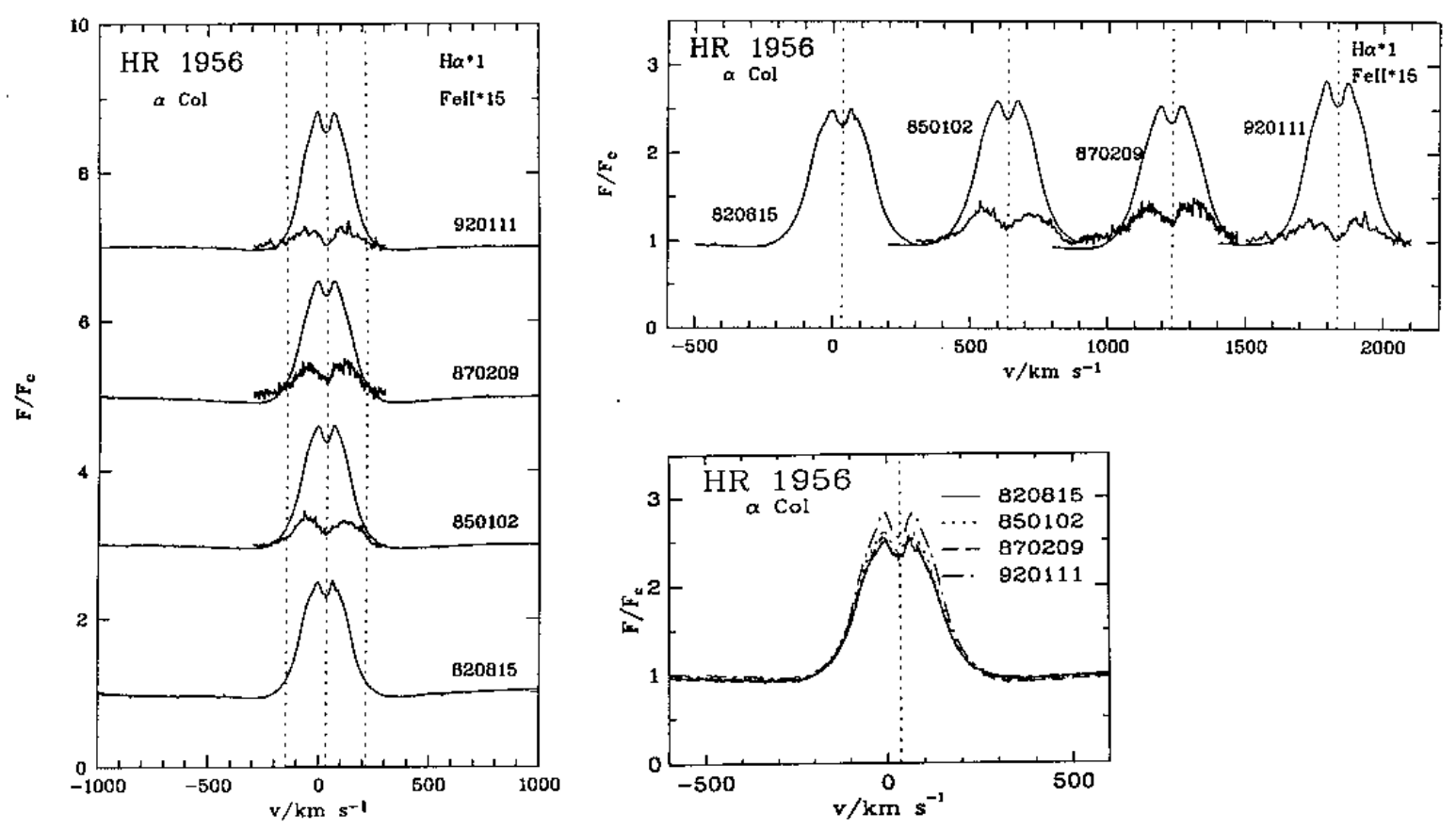

Fig. 19. $\mathrm{H} \alpha$ and $\mathrm{Fe}$ in profiles for $\mathrm{HR} 1956$
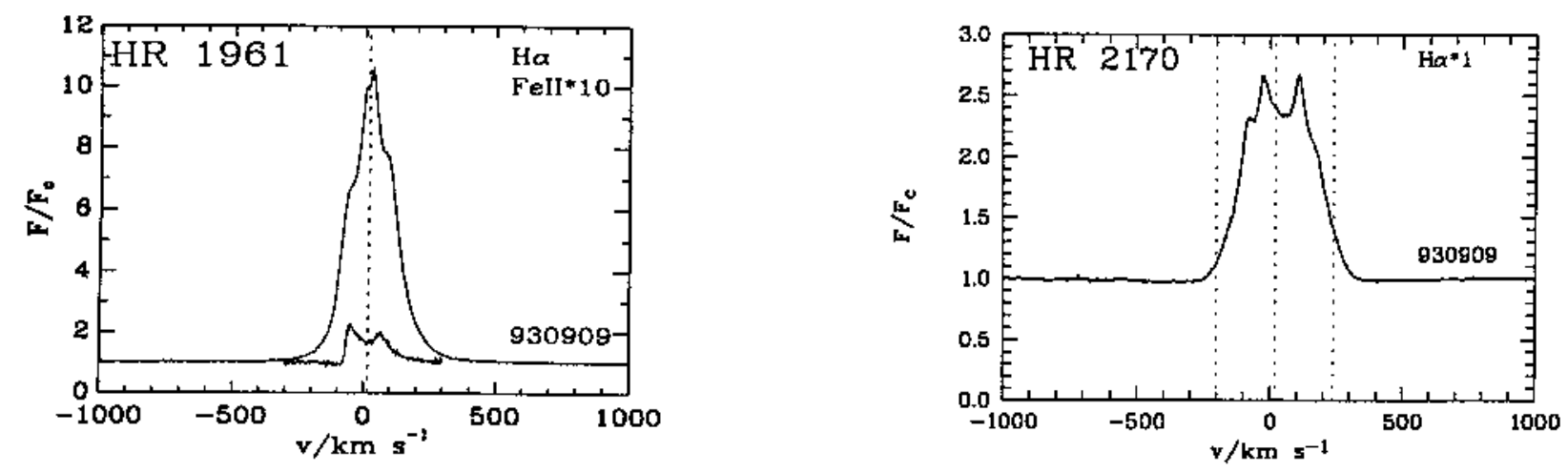

Fig. 20. $\mathrm{H} \alpha$ and Fe in profile for HR 1961

Fig. 21. $\mathrm{H} \alpha$ profile for HR 2170 

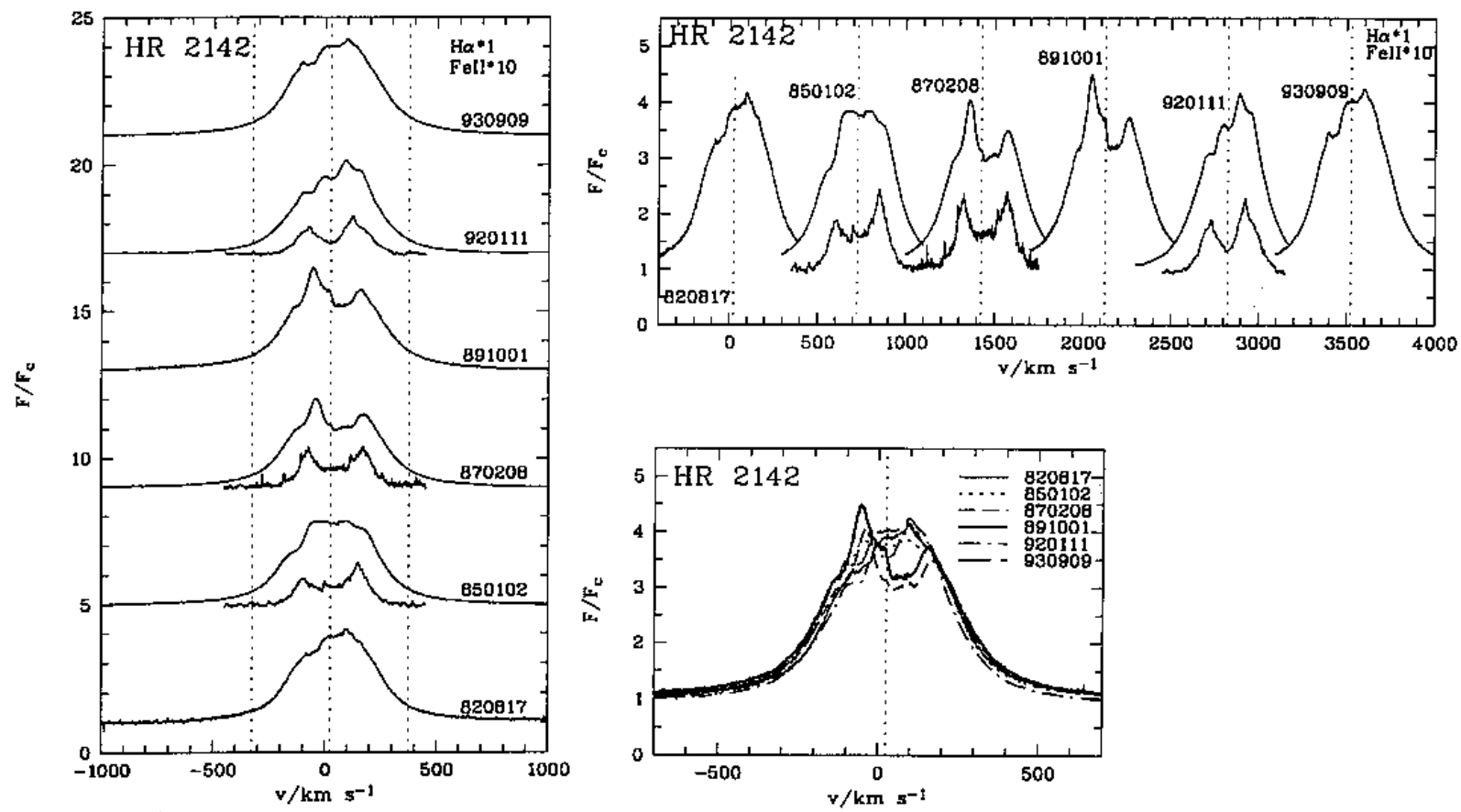

Fig. 22. $\mathrm{H} \alpha$ and Fe is profiles for HR 2142

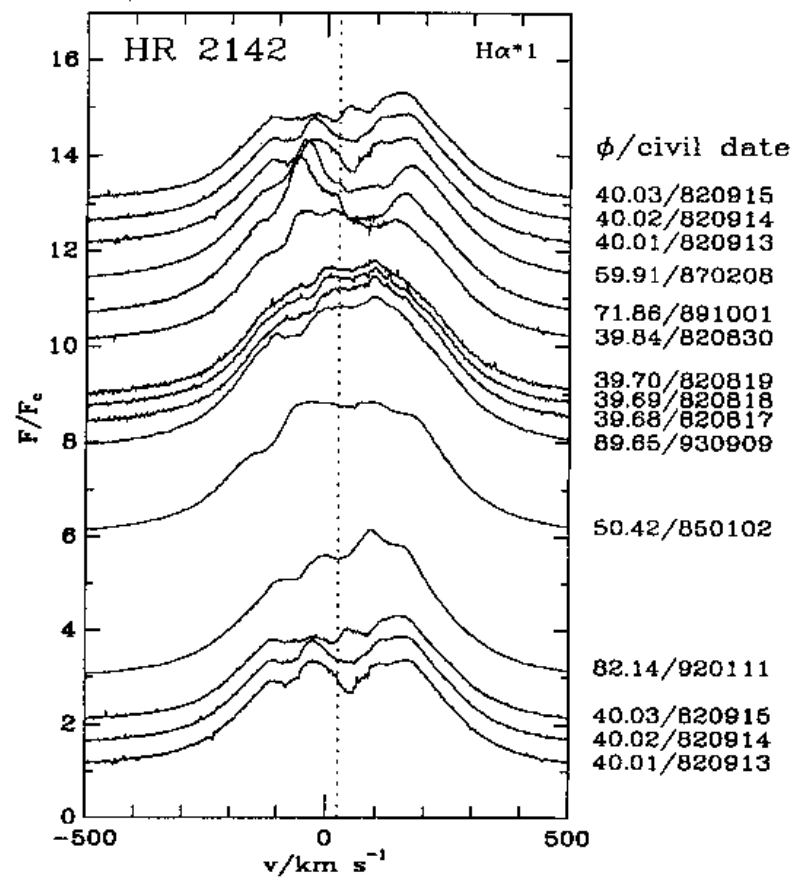

Fig. 23. Phase-binned H $\alpha$ profiles for HR 2142, using $P_{\text {orb }}=80.860$ days and $T_{0}=$ JD 2441990.5 (Peters 1983 ) 

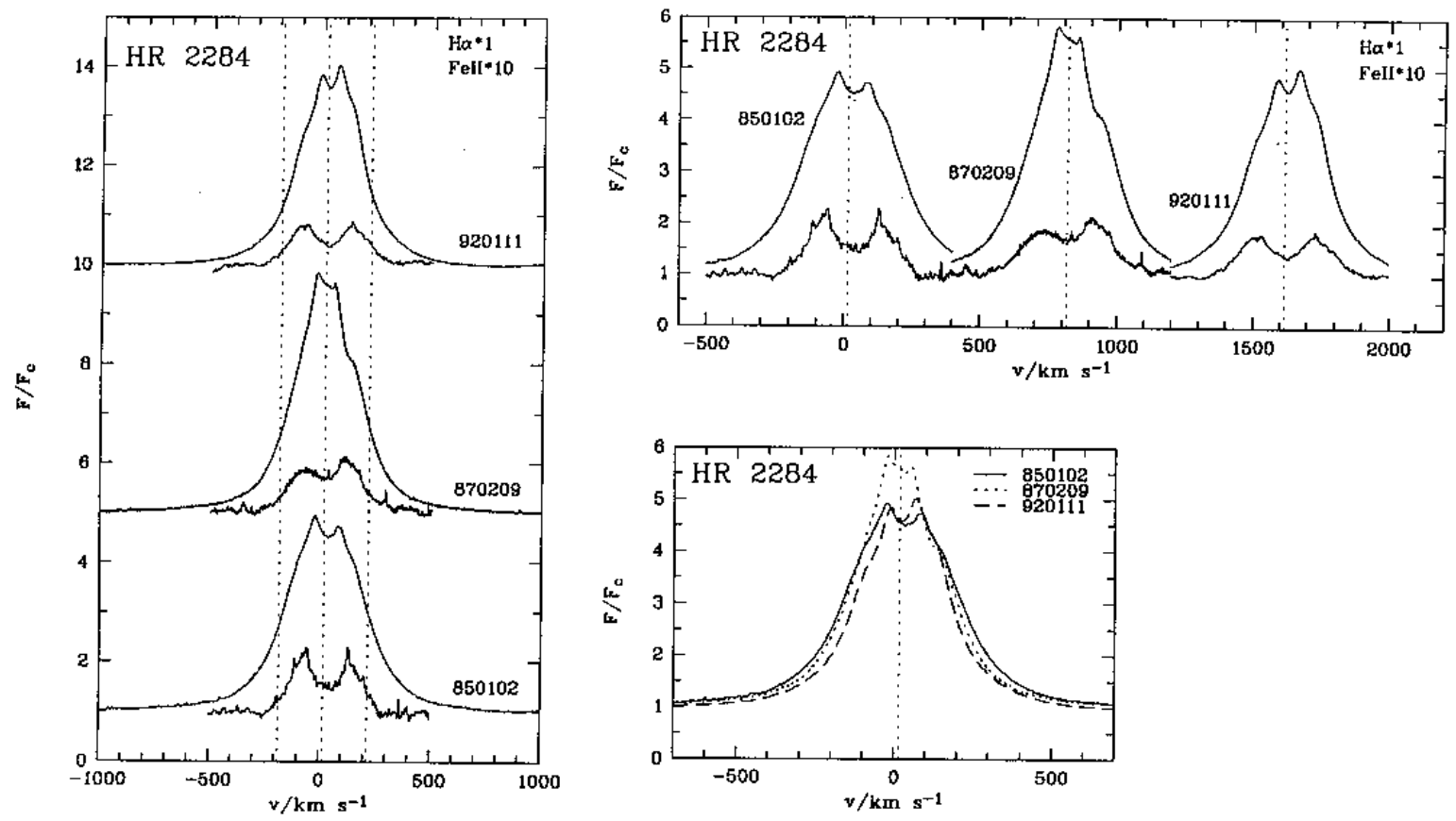

Fig. 24. $\mathrm{H} \alpha$ and $\mathrm{Fe}$ in profiles for $\mathrm{HR} 2284$
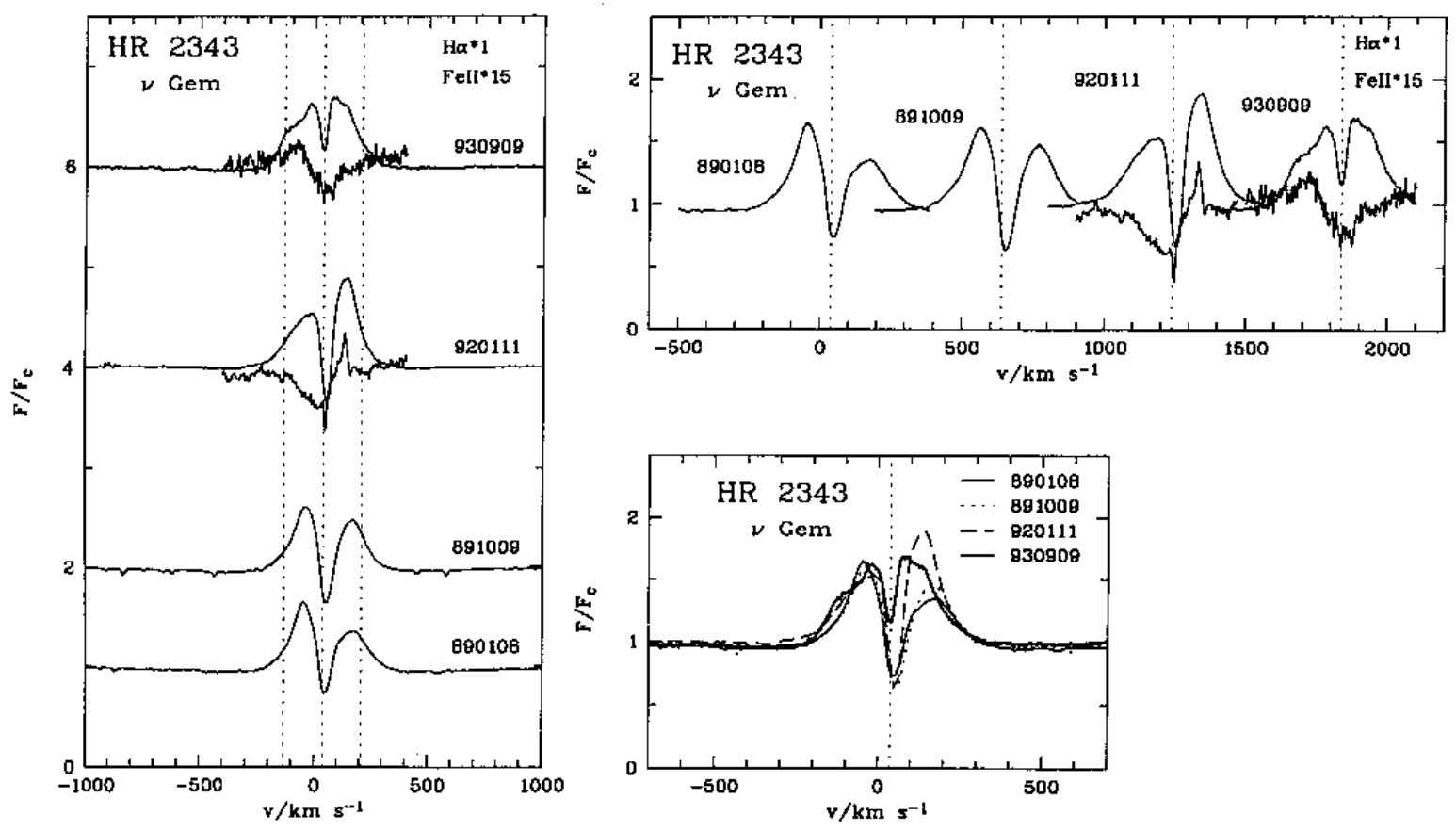

Fig. 25. H $\alpha$ and Fe in profiles for HR 2343. Note that by the expansion of the Fe in profiles also the shell troughs are affected 

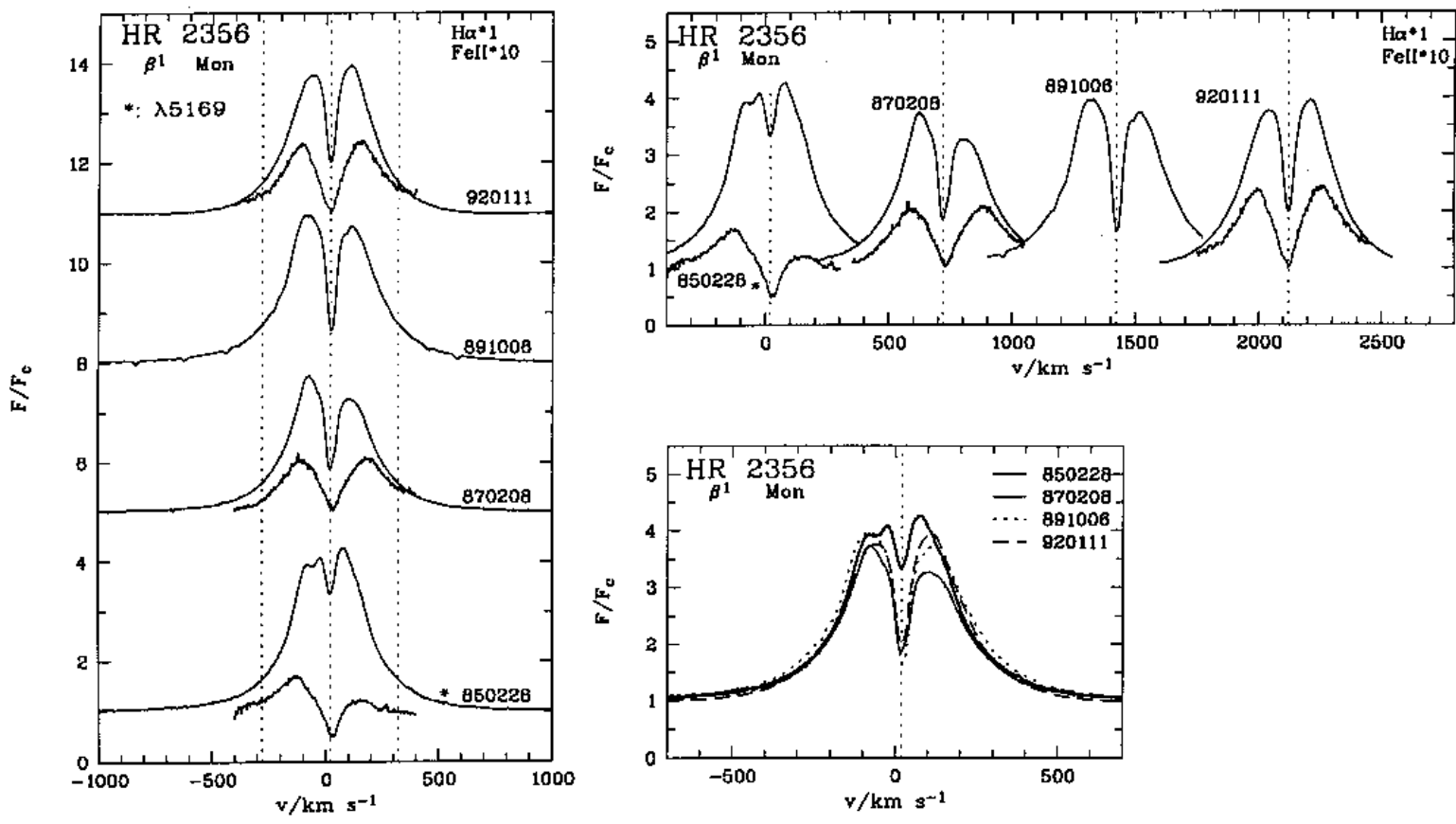

Fig. 26. $\mathrm{H} \alpha$ and Fe II profiles for HR 2356. In 1985, the Fe II $\lambda 5169$ line has been measured instead of $\lambda 5317$ (marked by an asterisk)
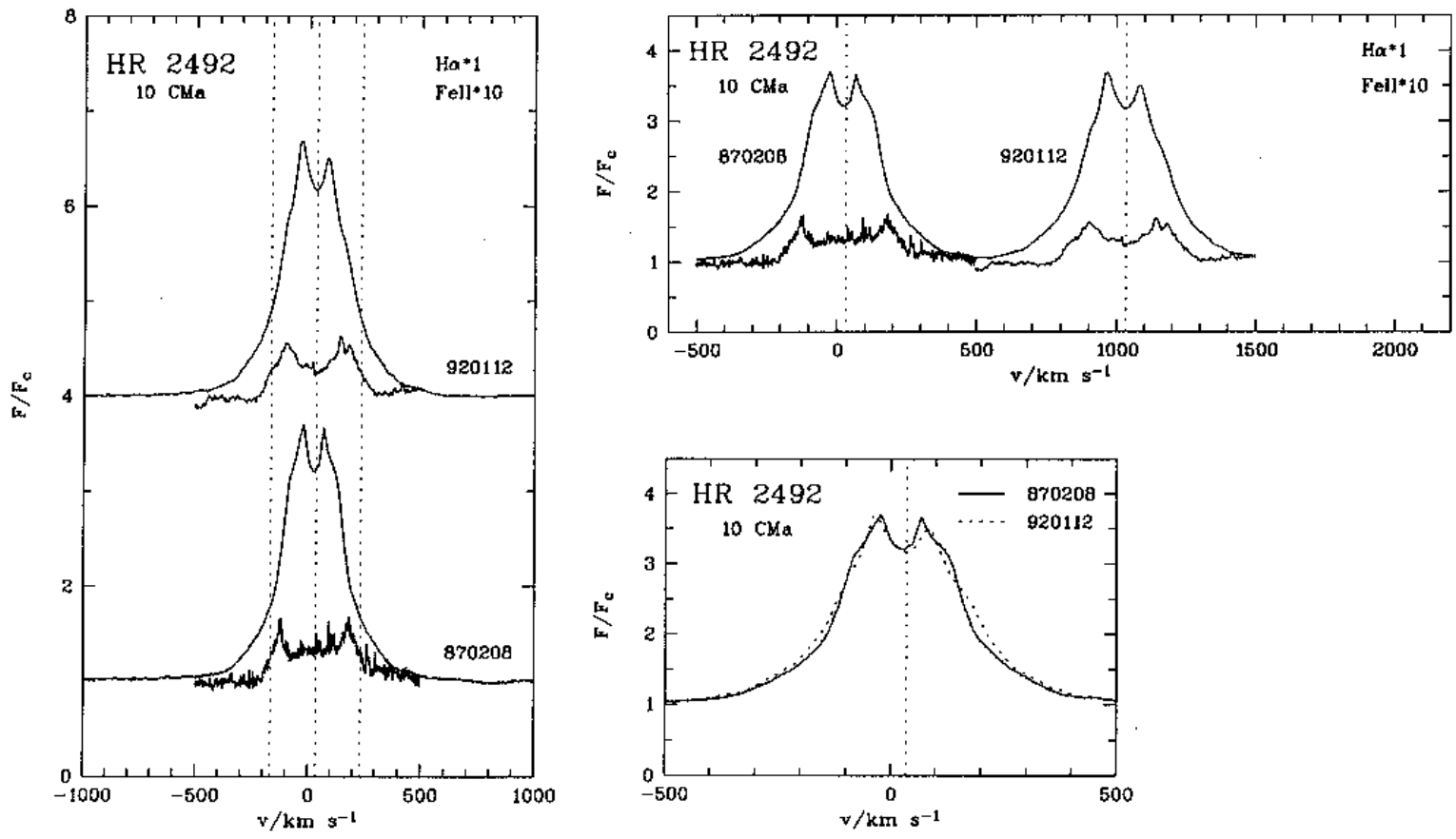

Fig. 27. $\mathrm{H} \alpha$ and Fe in profiles for $\mathrm{HR} 2492$ 

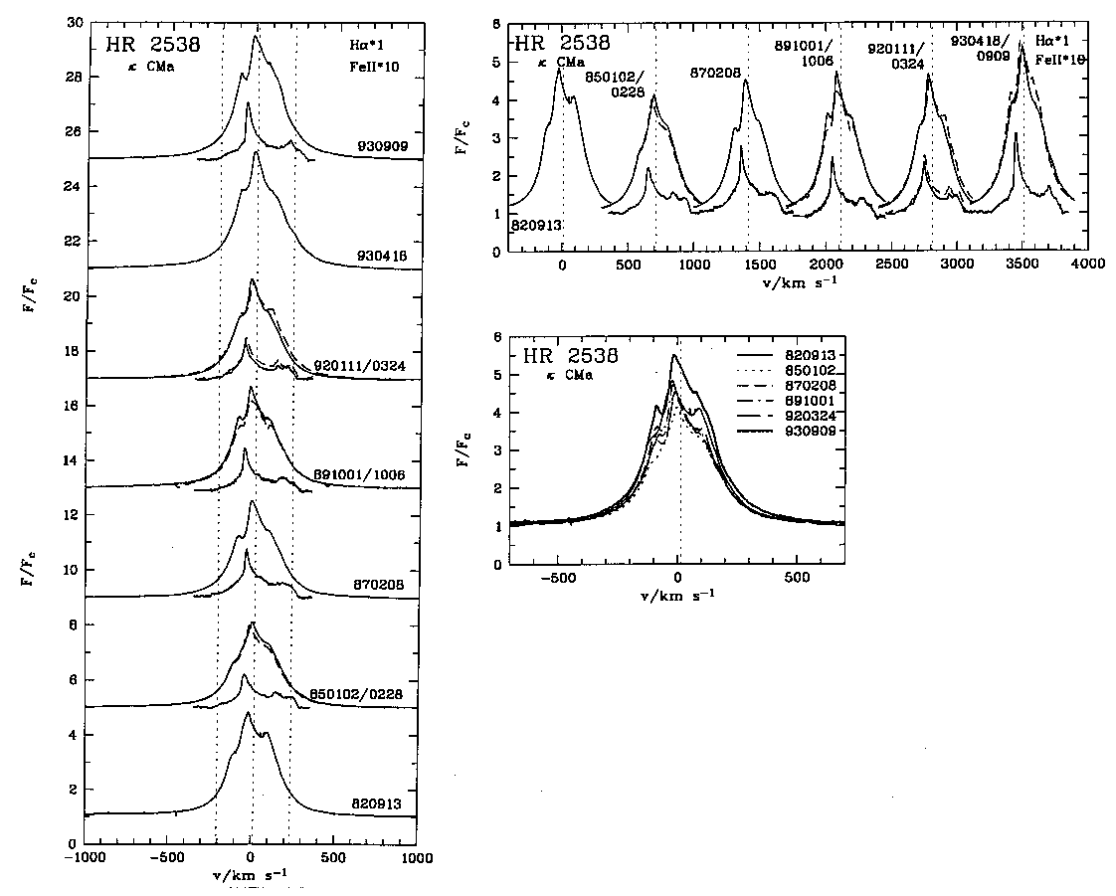

Fig. 28. $\mathrm{H} \alpha$ and $\mathrm{Fe}$ in profiles for $\mathrm{HR} 2538$
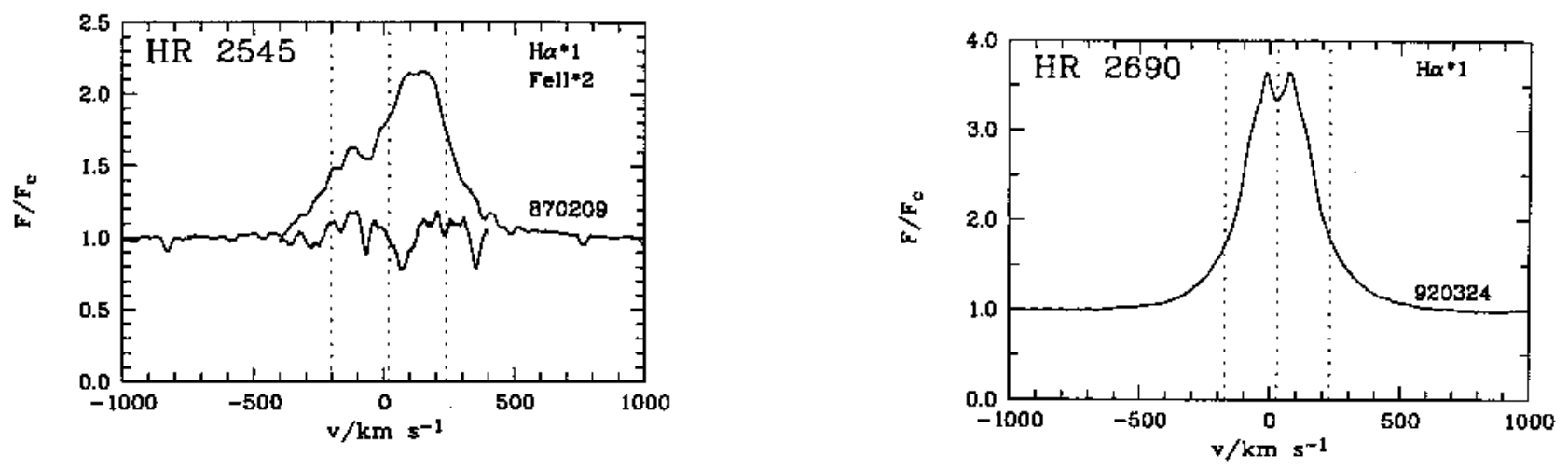

Fig. 29. H $\alpha$ and Fe II profile for HR 2545; the A-type companion star produces the narrow absorption features

Fig. 30. $\mathrm{H} \alpha$ profile for $\mathrm{HR} 2690$ 


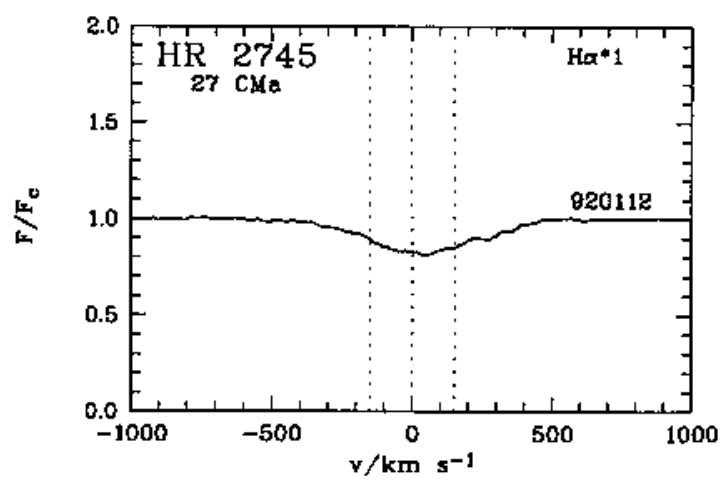

Fig. 31. $\mathrm{H} \alpha$ profile for $\mathrm{HR} 2745$
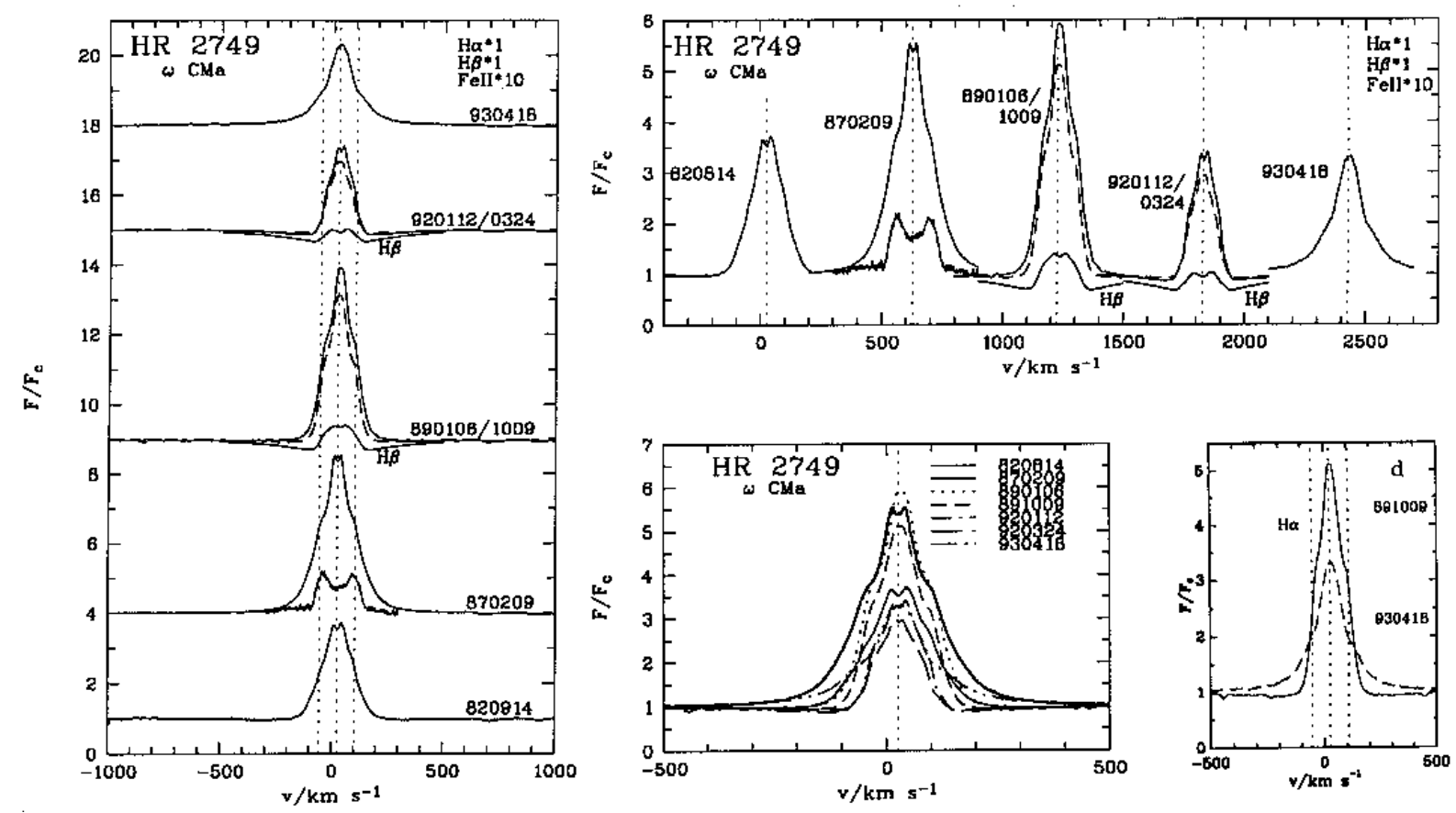

Fig. 32. $\mathrm{H} \alpha, \mathrm{H} \beta$ and Fe II profiles for HR 2749. Panel d demonstrates the dramatical change in width and height of the H $\alpha$ line between 1989 and 1992 

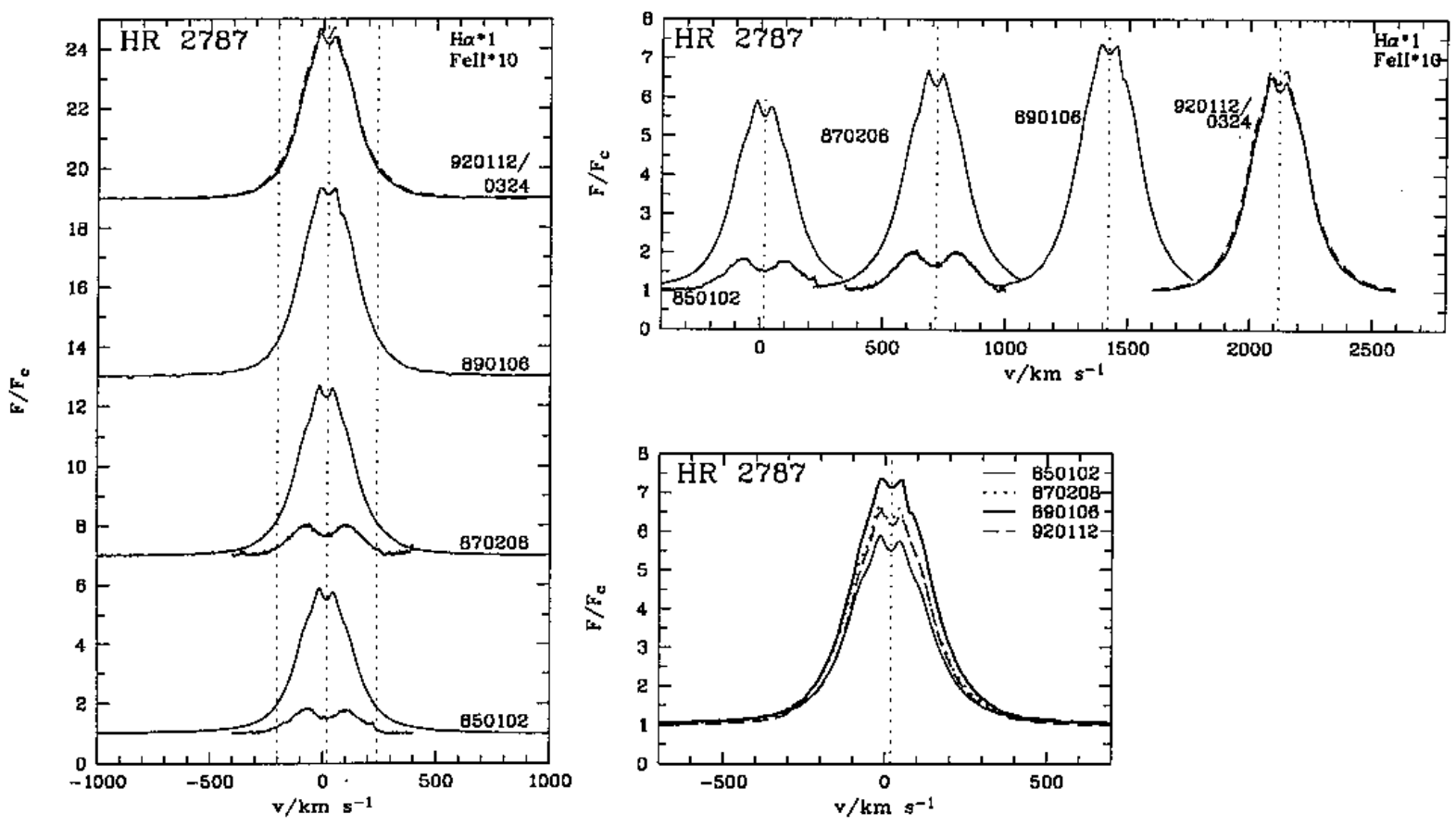

Fig. 33. $\mathrm{H} \alpha$ and Fe in profiles for $\mathrm{HR} 2787$
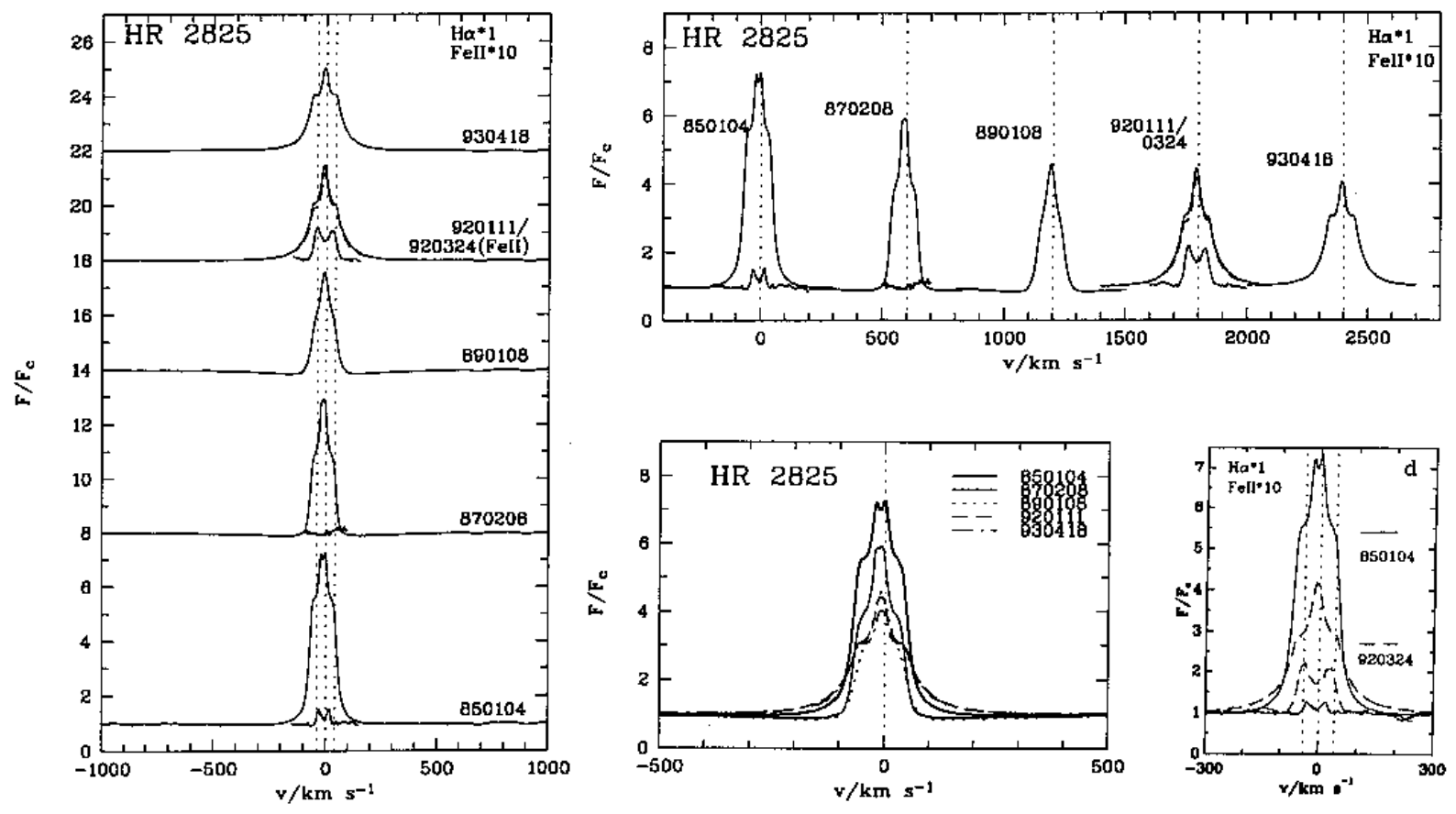

Fig. 34. $\mathrm{H} \alpha$ and $\mathrm{Fe}$ in profiles for $\mathrm{HR}$ 2825. The $\mathrm{H} \alpha$ profile from 1985 is the only one known to us showing peak splitting due to NSB broadening. Panel d shows the variation of line width and intensity both of $\mathrm{H} \alpha$ and Fe II (anti-correlation) 

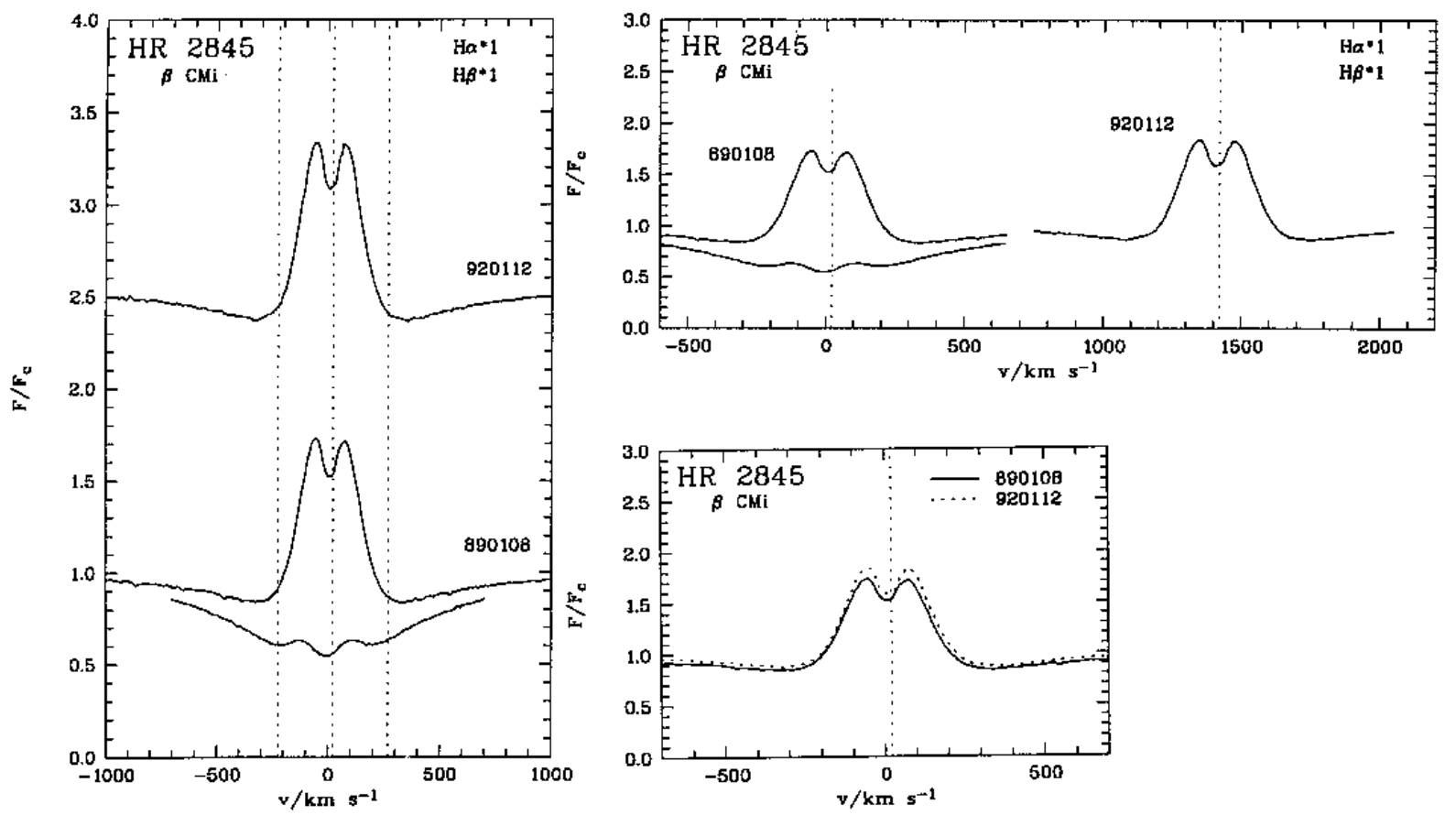

Fig. 35. $\mathrm{H} \alpha$ and $\mathrm{H} \beta$ profiles for $\mathrm{HR} 2845$
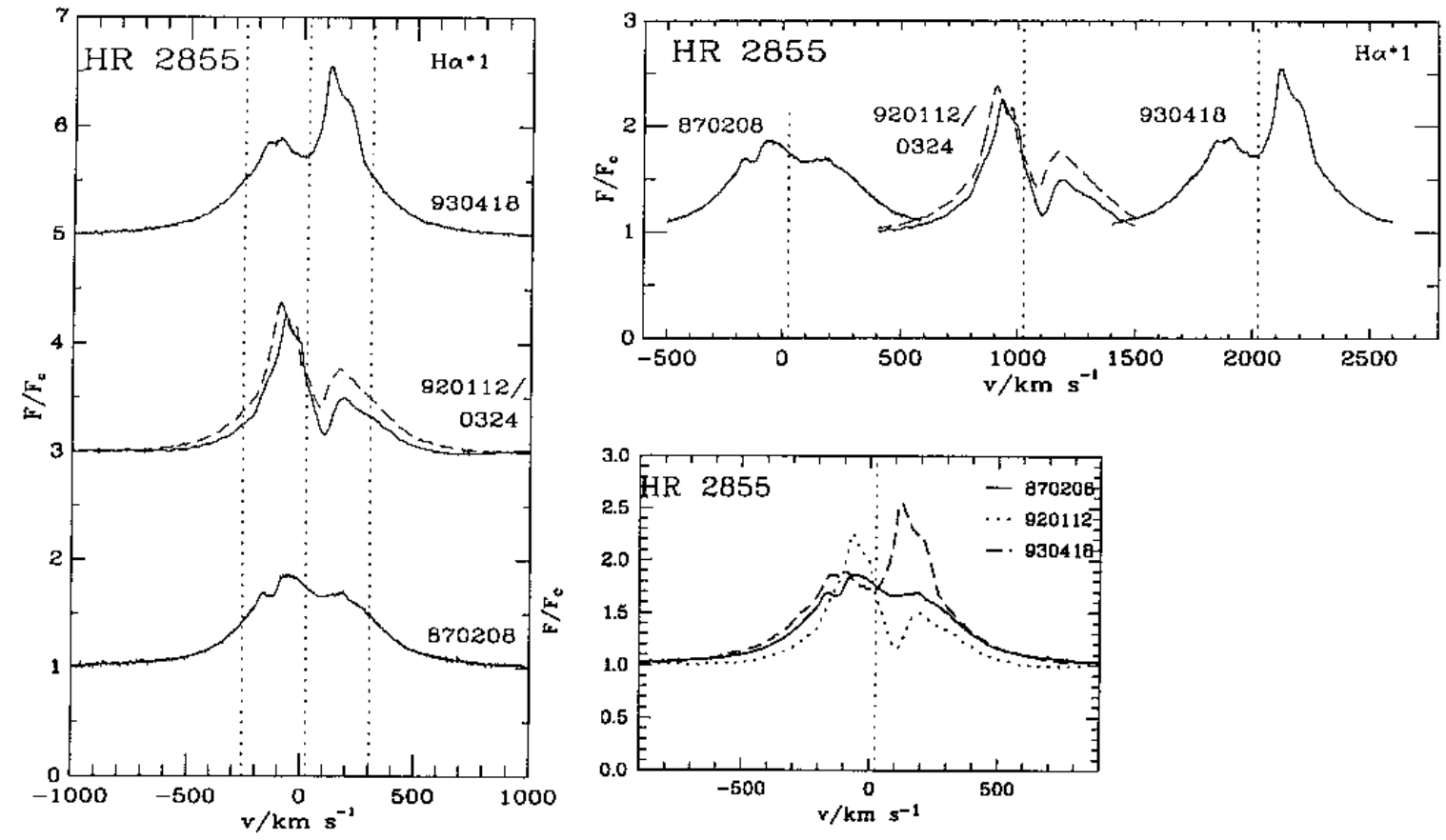

Fig. 36. H $\alpha$ profiles for $\mathrm{HR} 2855$ (Fe II emission was present in 1992, but profile has been lost) 

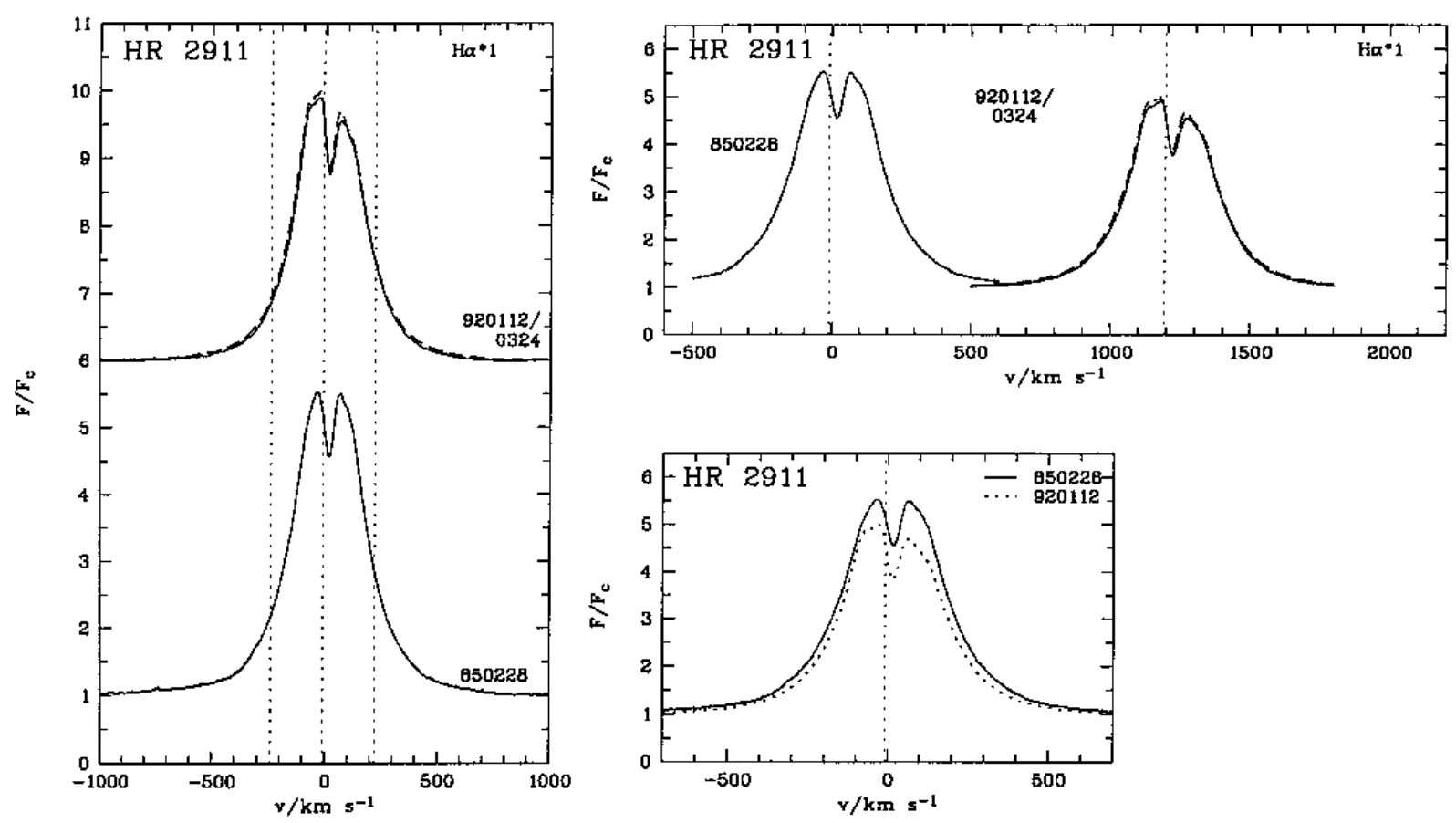

Fig. 37. $\mathrm{H} \alpha$ profiles for $\mathrm{HR} 2911$

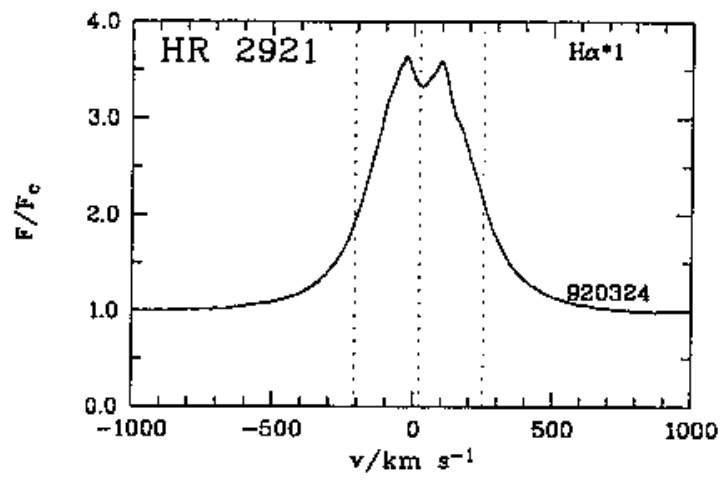

Fig. 38. $\mathrm{H} \alpha$ profile for $\mathrm{HR} 2921$ 

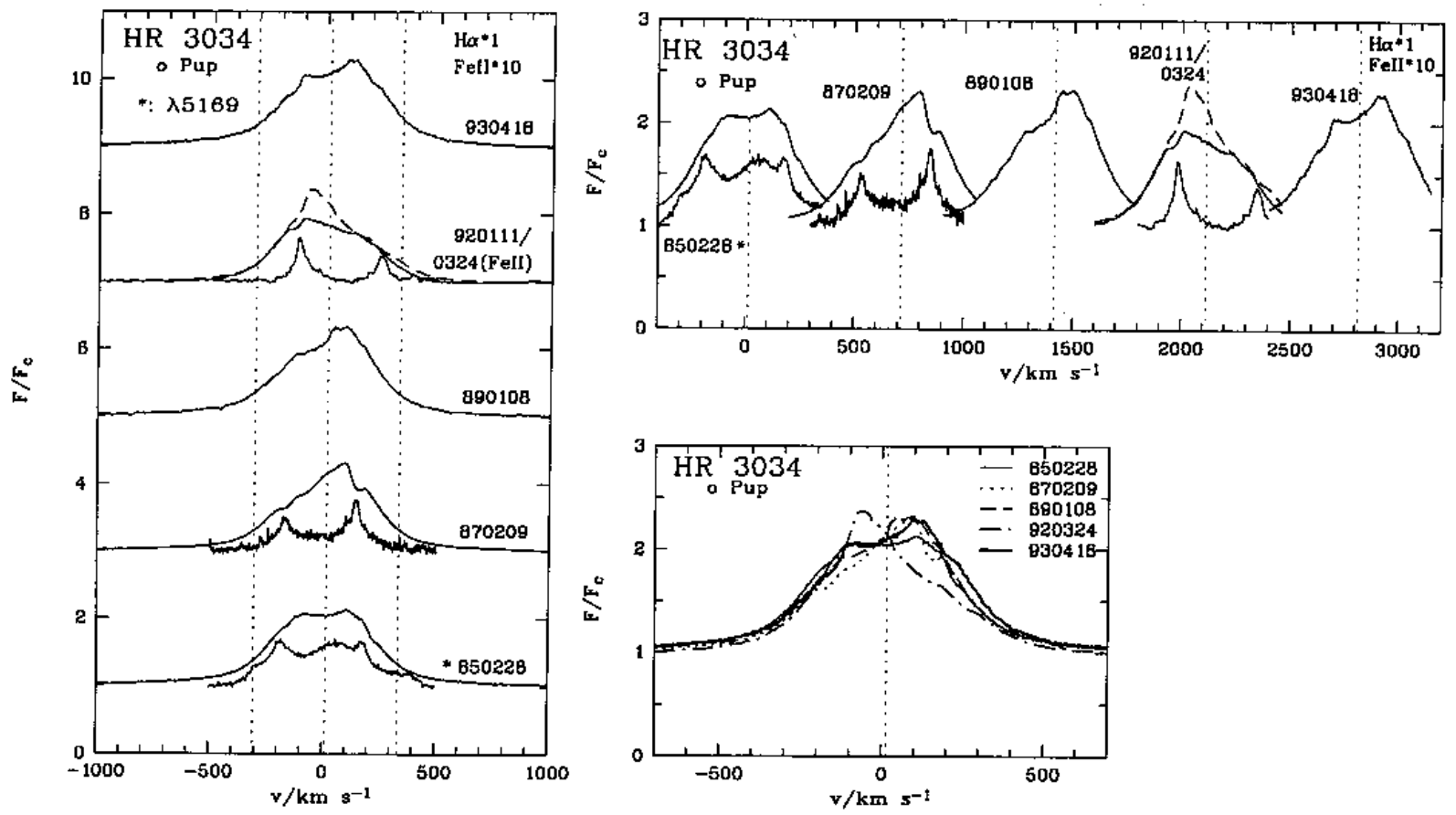

Fig. 39. $\mathrm{H} \alpha$ and Fe in profiles for HR 3034. The complex structure of the Fe in $\lambda 5169$ line is due to faint blends
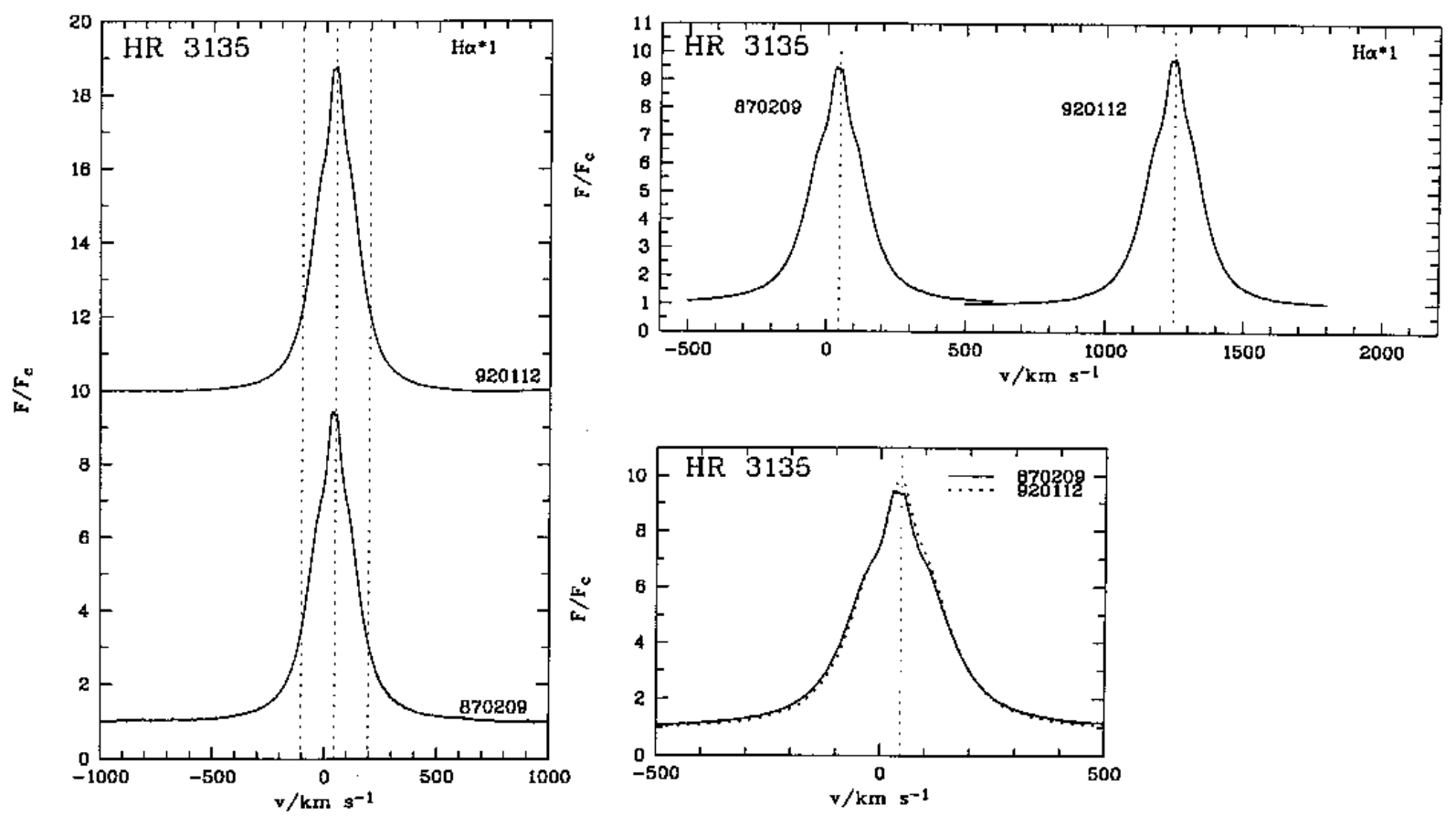

Fig. 40. $\mathrm{H} \alpha$ profiles for $\mathrm{HR} 3135$ 


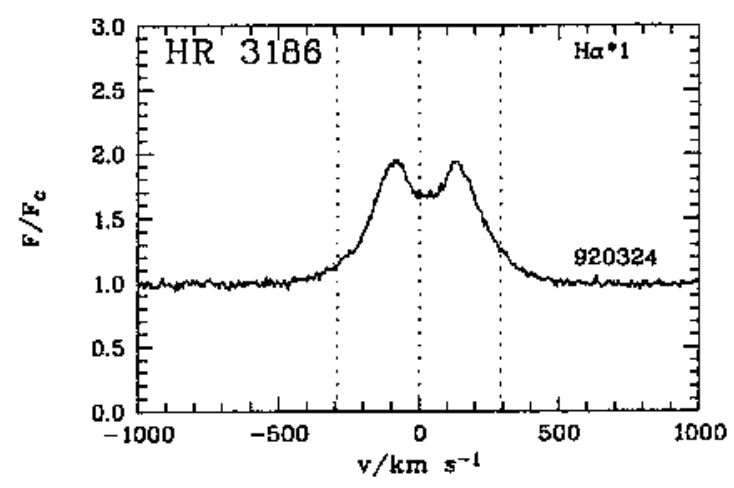

Fig. 41. $\mathrm{H} \alpha$ profile for $\mathrm{HR} 3186$
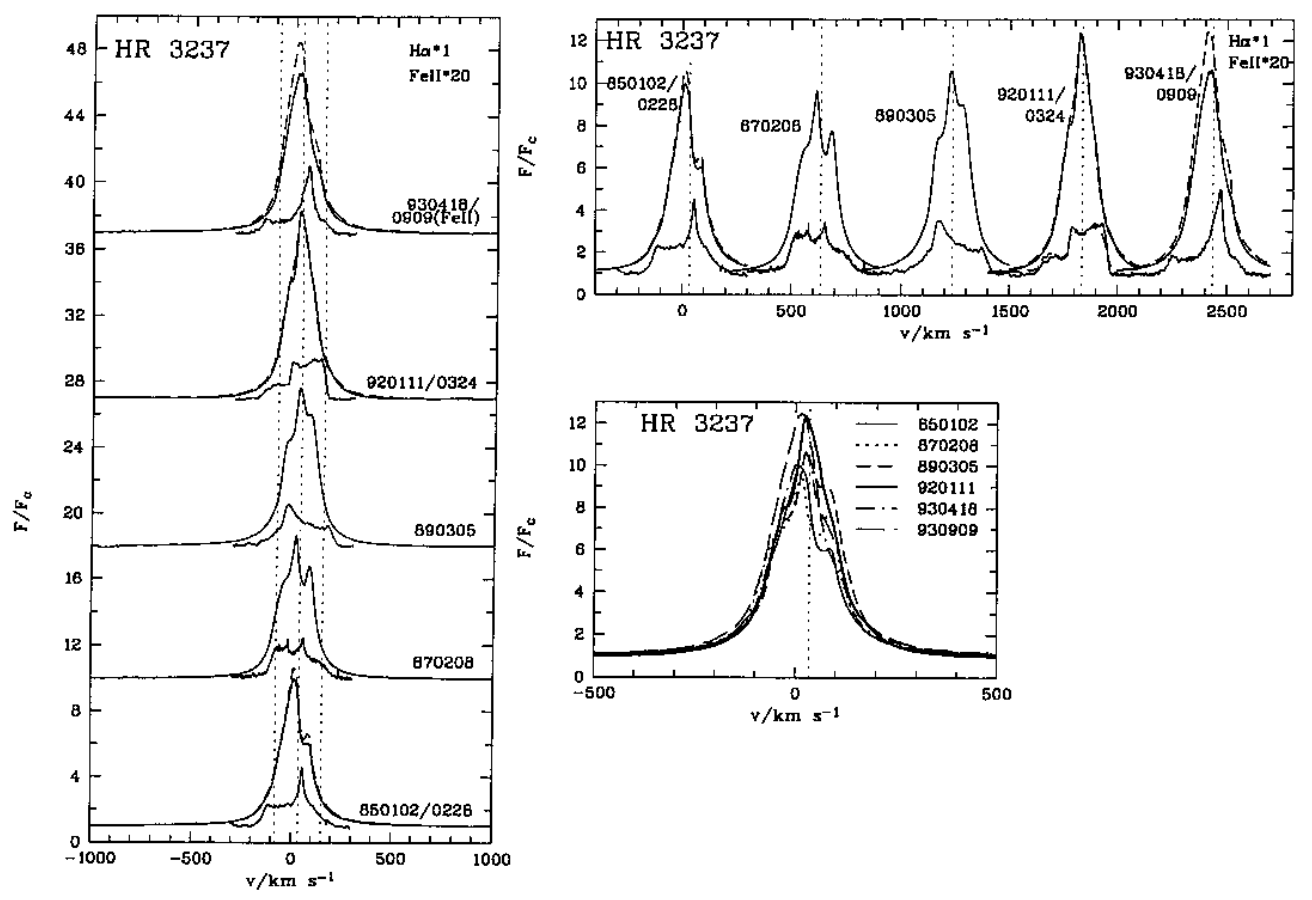

Fig. 42. $\mathrm{H} \alpha$ and $\mathrm{Fe}$ in profiles for $\mathrm{HR} 3237$ 

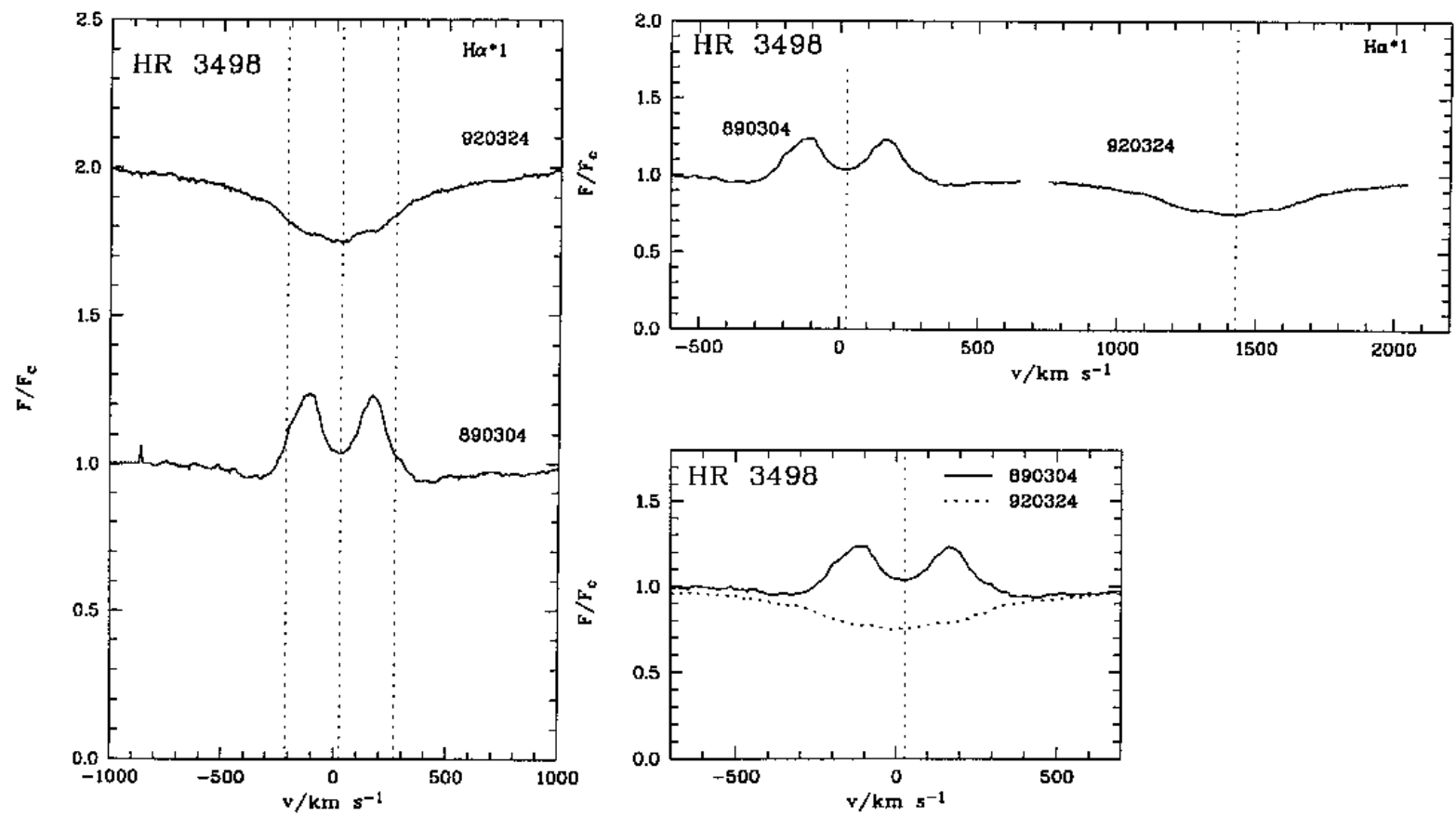

Fig. 43. $\mathrm{H} \alpha$ profiles for HR 3498
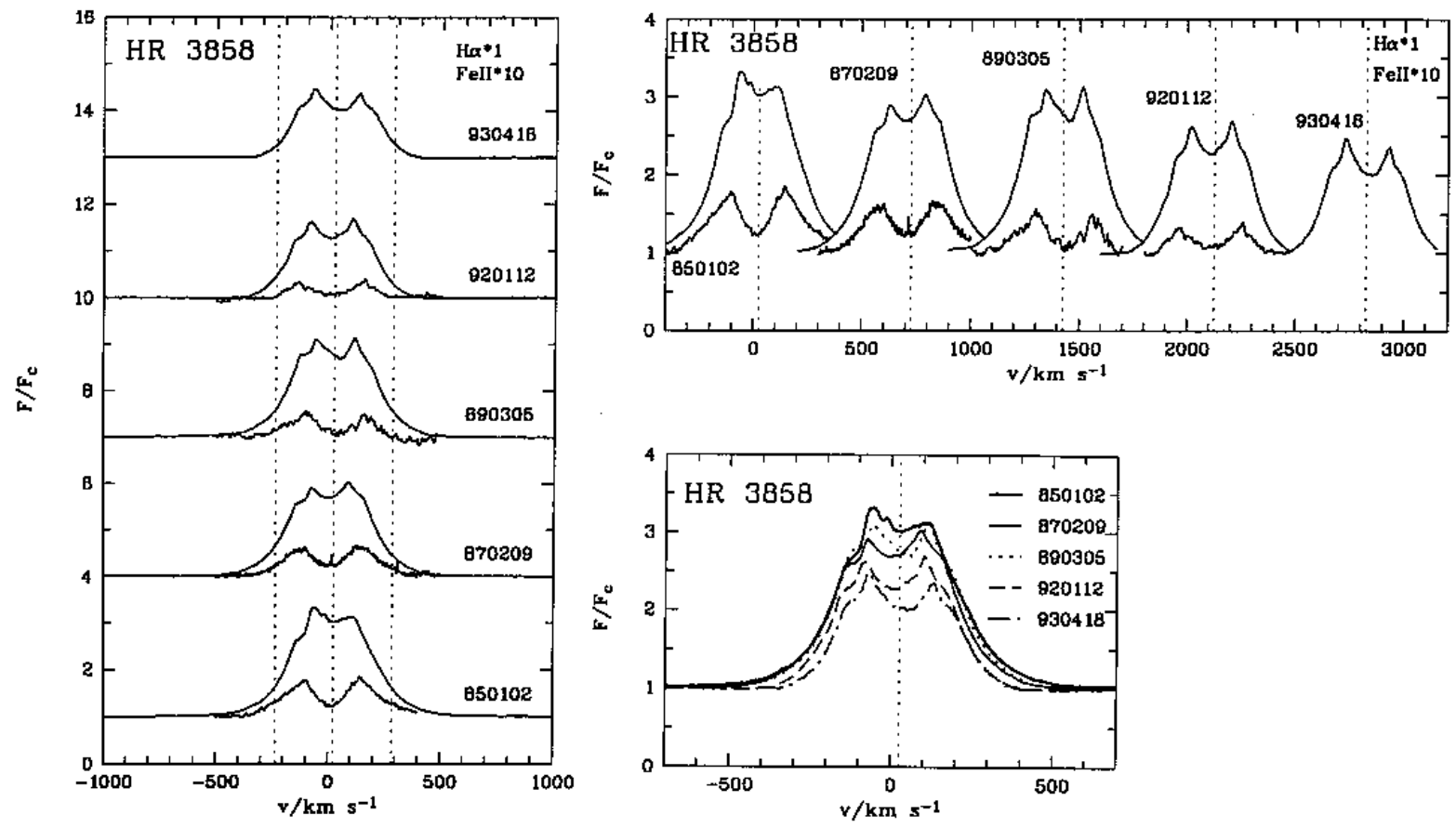

Fig. 44. $\mathrm{H} \alpha$ and Fe II profiles for HR 3858 

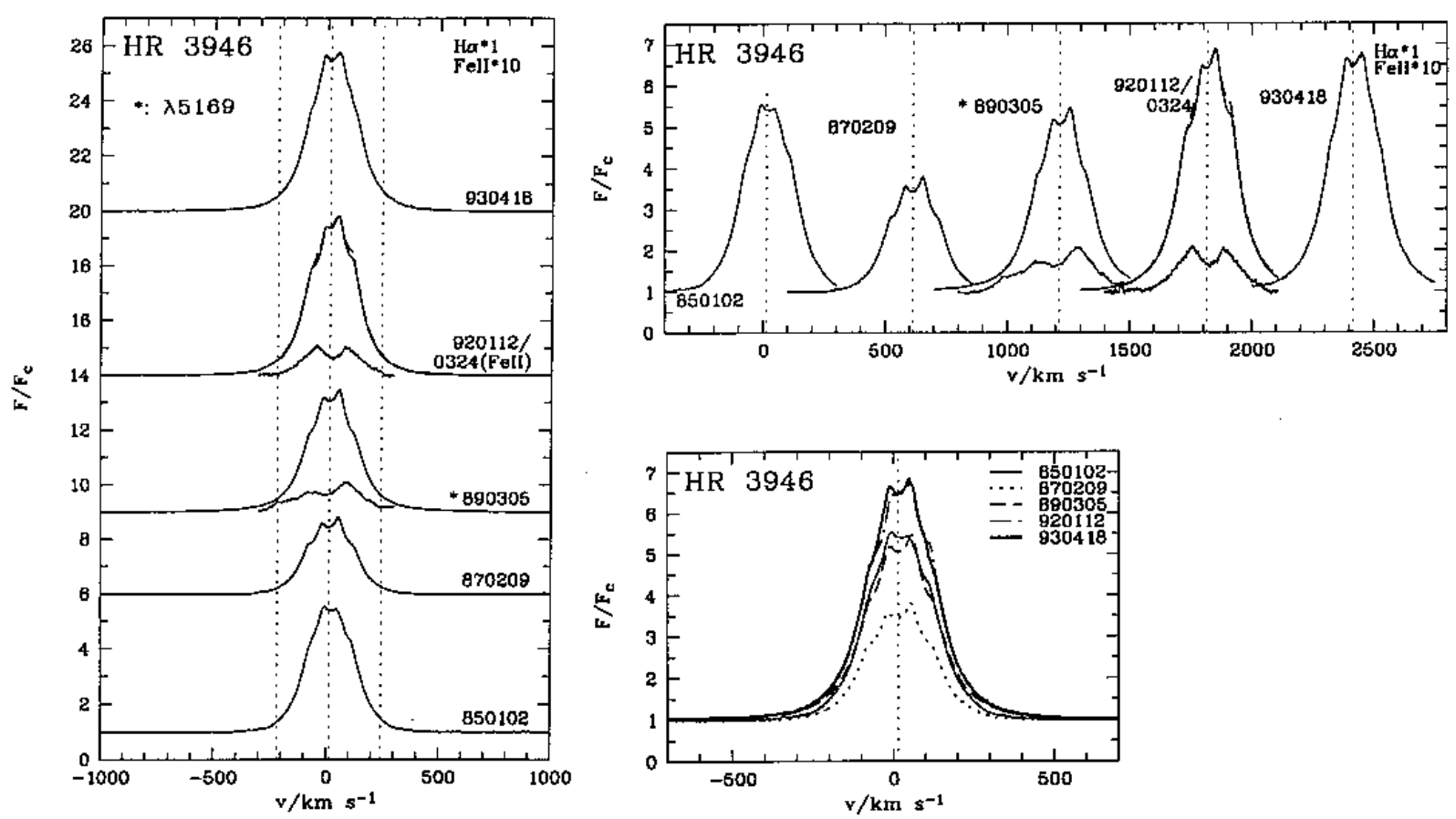

Fig. 45. $\mathrm{H} \alpha$ and $\mathrm{Fe}$ in profiles for $\mathrm{HR} 3946$
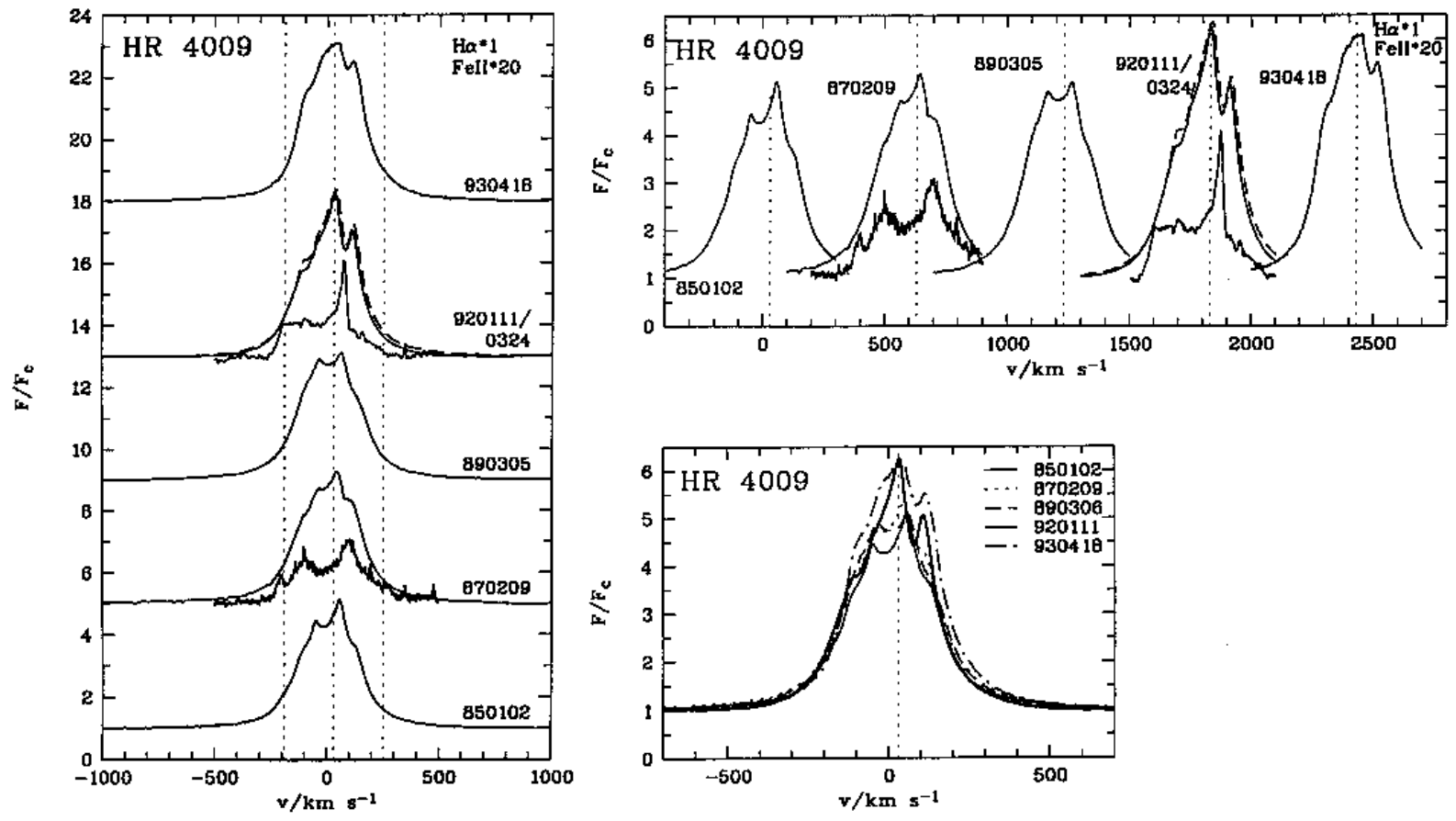

Fig. 46. $\mathrm{H} \alpha$ and $\mathrm{Fe}$ in profiles for $\mathrm{HR} 4009$ 


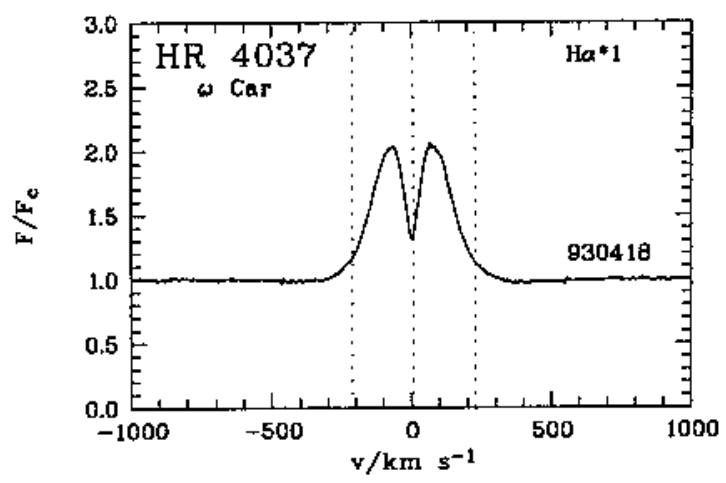

Fig. 47. $\mathrm{H} \alpha$ profile for $\mathrm{HR} 4037$
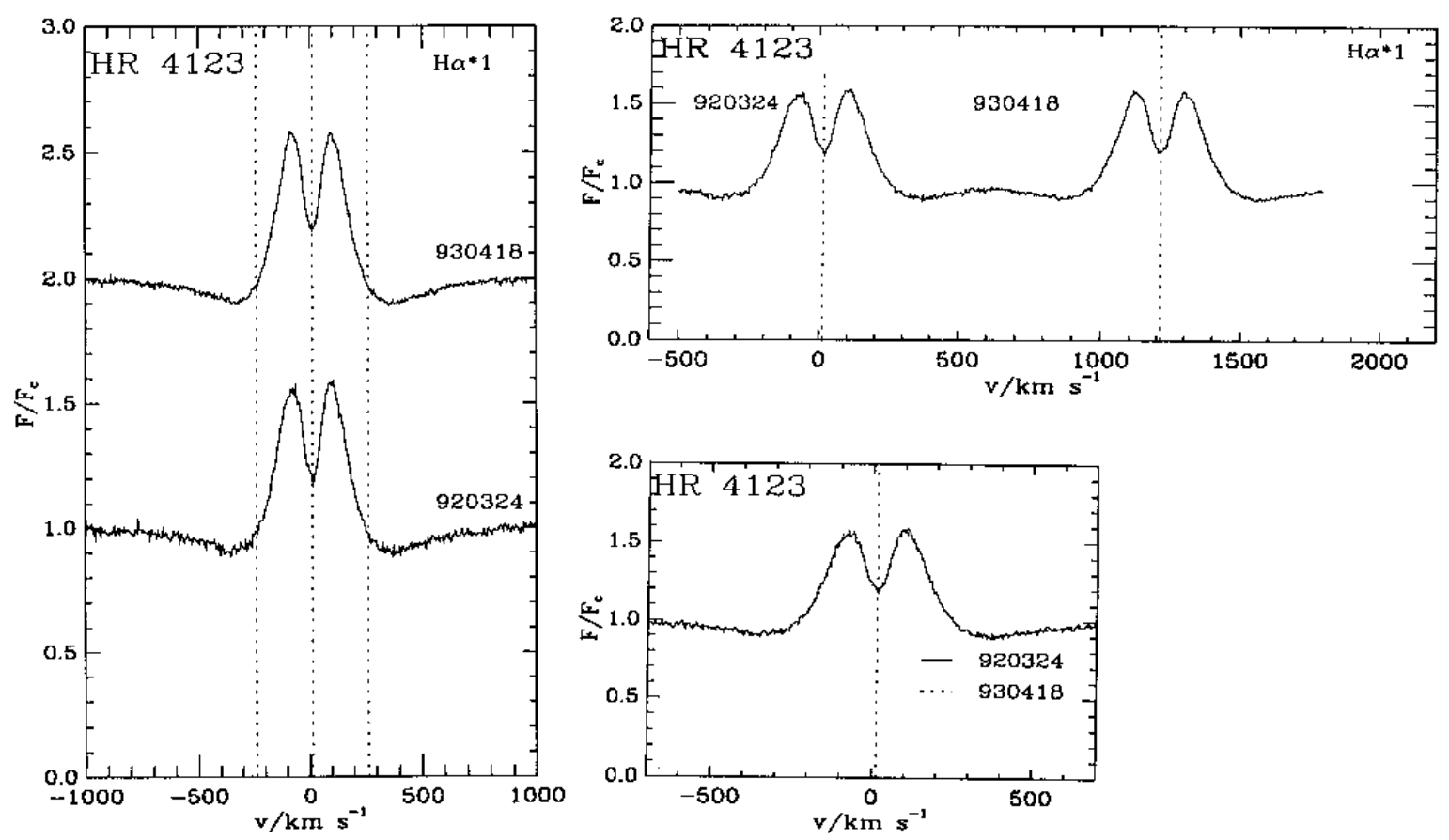

Fig. 48. $\mathrm{H} \alpha$ profiles for $\mathrm{HR}$ 4123. The two $\mathrm{H} \alpha$ profiles almost fall together in panel c 

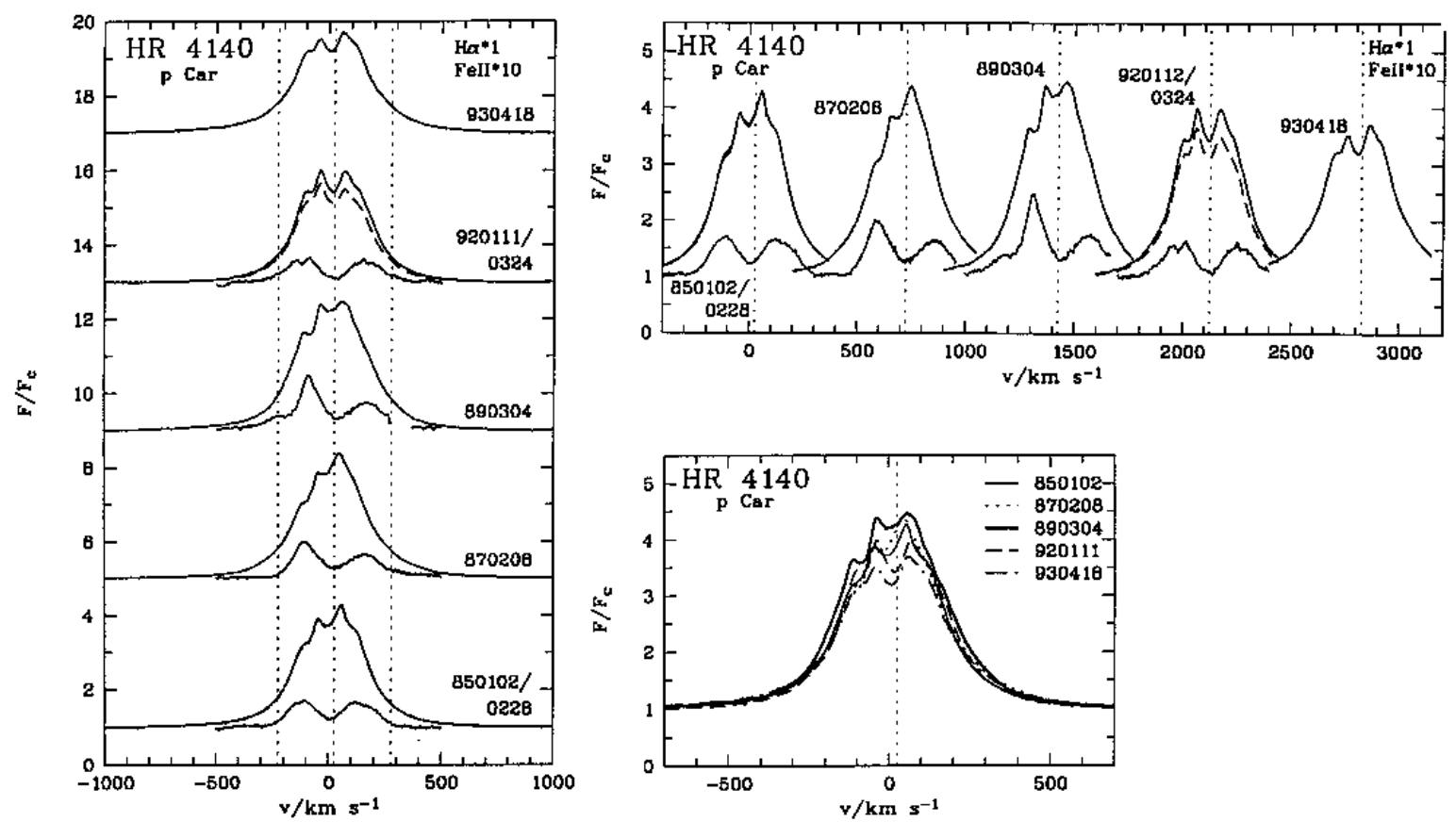

Fig. 49. $\mathrm{H} \alpha$ and $\mathrm{Fe}$ in profiles for $\mathrm{HR} 4140$
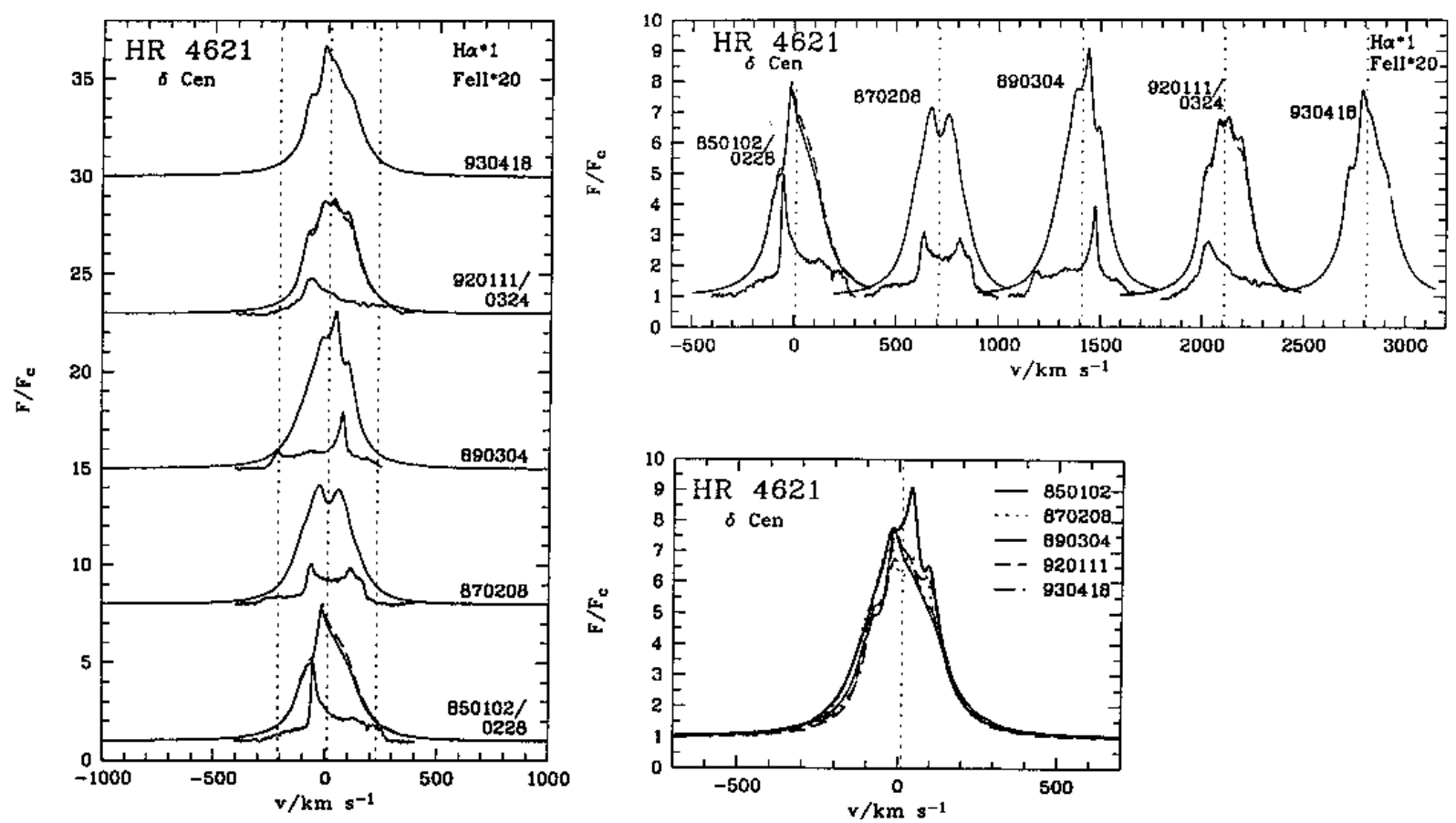

Fig. 50. $\mathrm{H} \alpha$ and Fe in profiles for HR 4621. Note the prototype "steeple" shape of the Fe in profiles in 1985 and 1989 

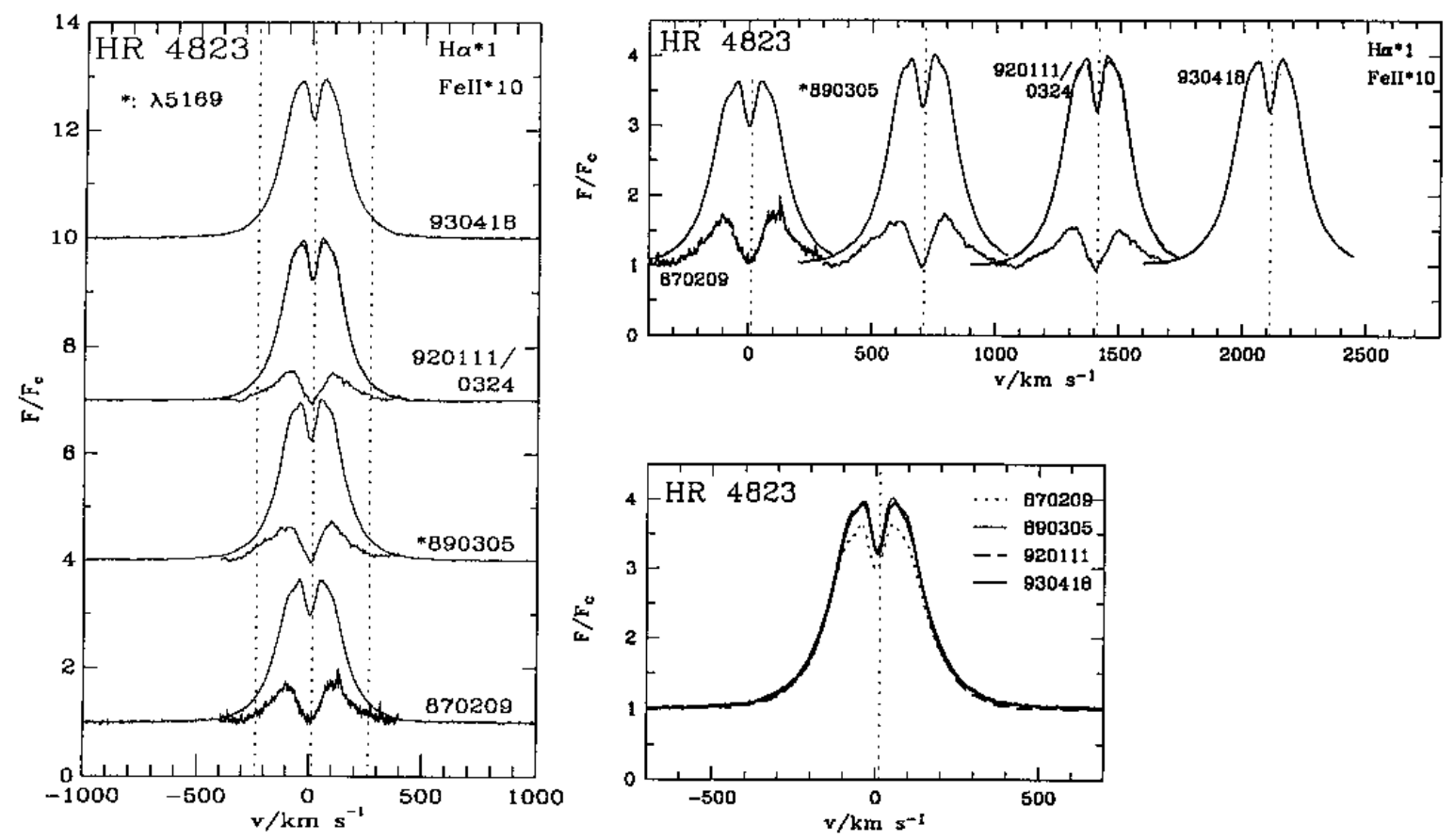

Fig. 51. $\mathrm{H} \alpha$ and $\mathrm{Fe}$ in profiles for $\mathrm{HR} 4823$
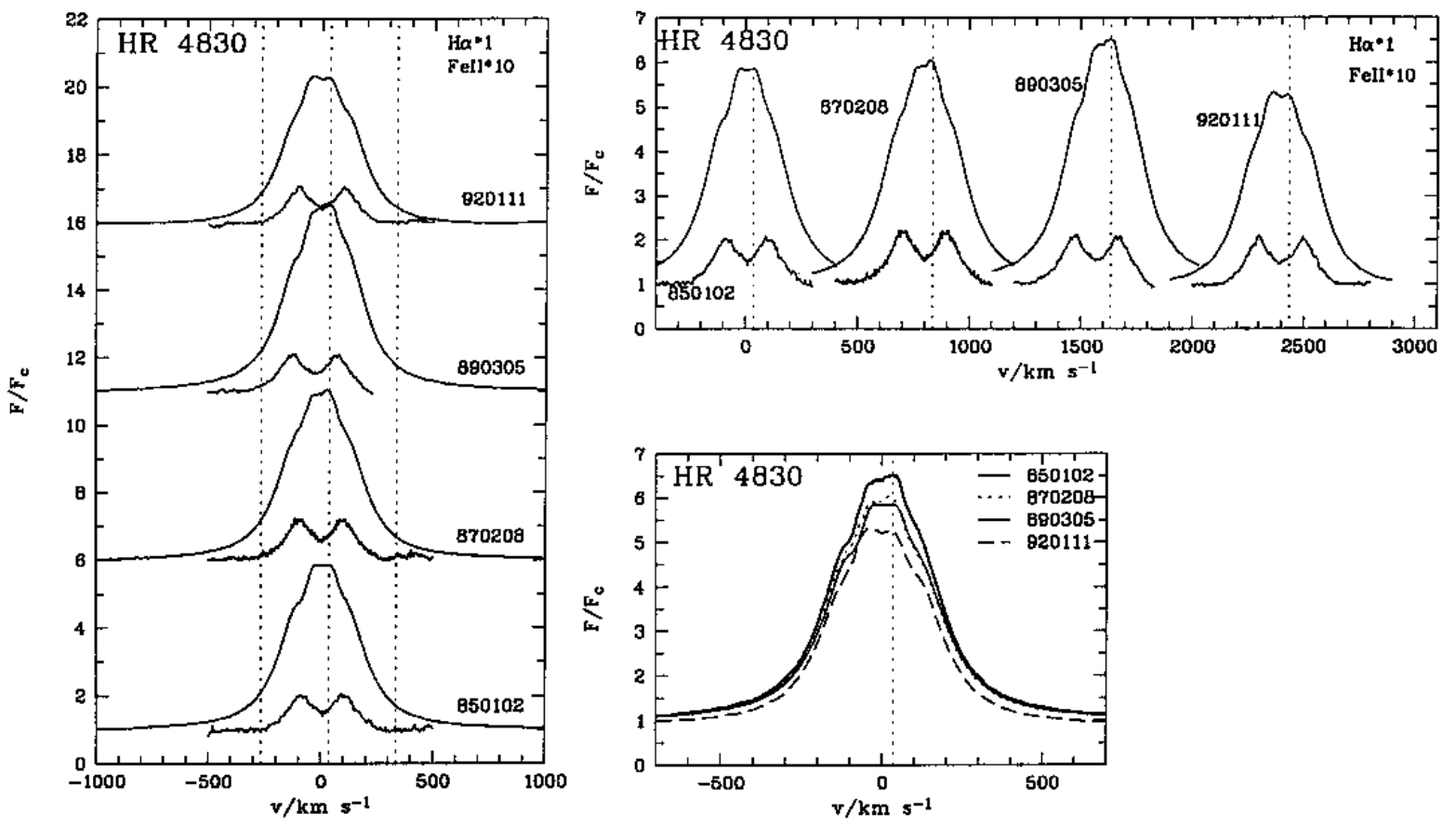

Fig. 52. $\mathrm{H} \alpha$ and $\mathrm{Fe}$ in profiles for $\mathrm{HR} 4830$ 


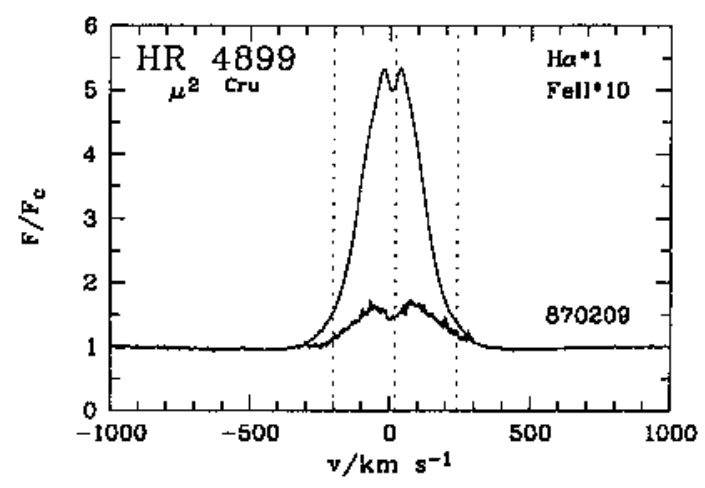

Fig. 53. H $\alpha$ and Fe iा profile for HR 4899 (a further Fe II measurement in 920324 did not yield any emission)
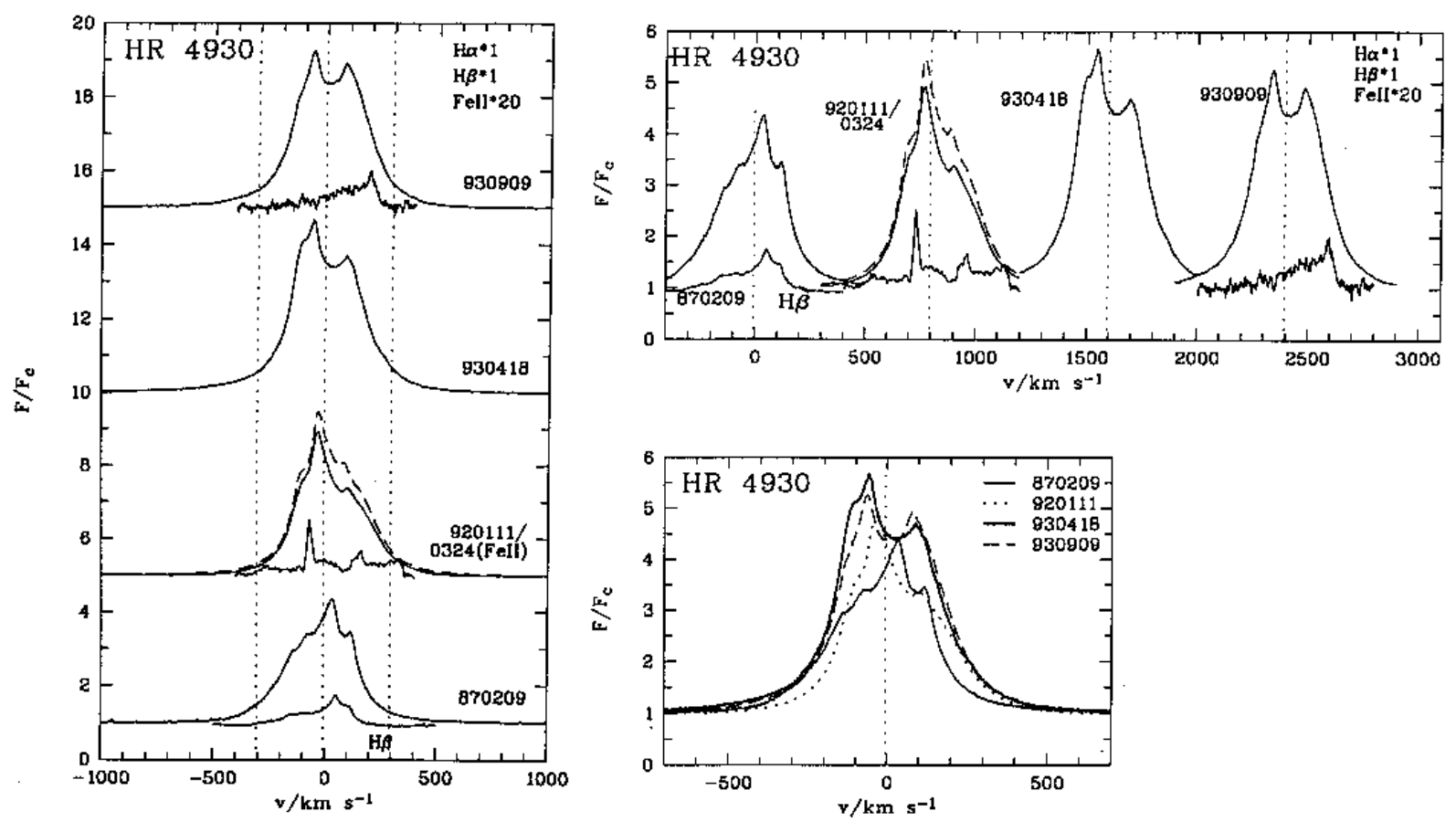

Fig. 54. $\mathrm{H} \alpha, \mathrm{H} \beta$ and $\mathrm{Fe}$ in profiles for $\mathrm{HR}$ 4930. Note that the complex substructure in the 1992 Fe II profile might be spurious (within the uncertainty of the normalization procedure) 

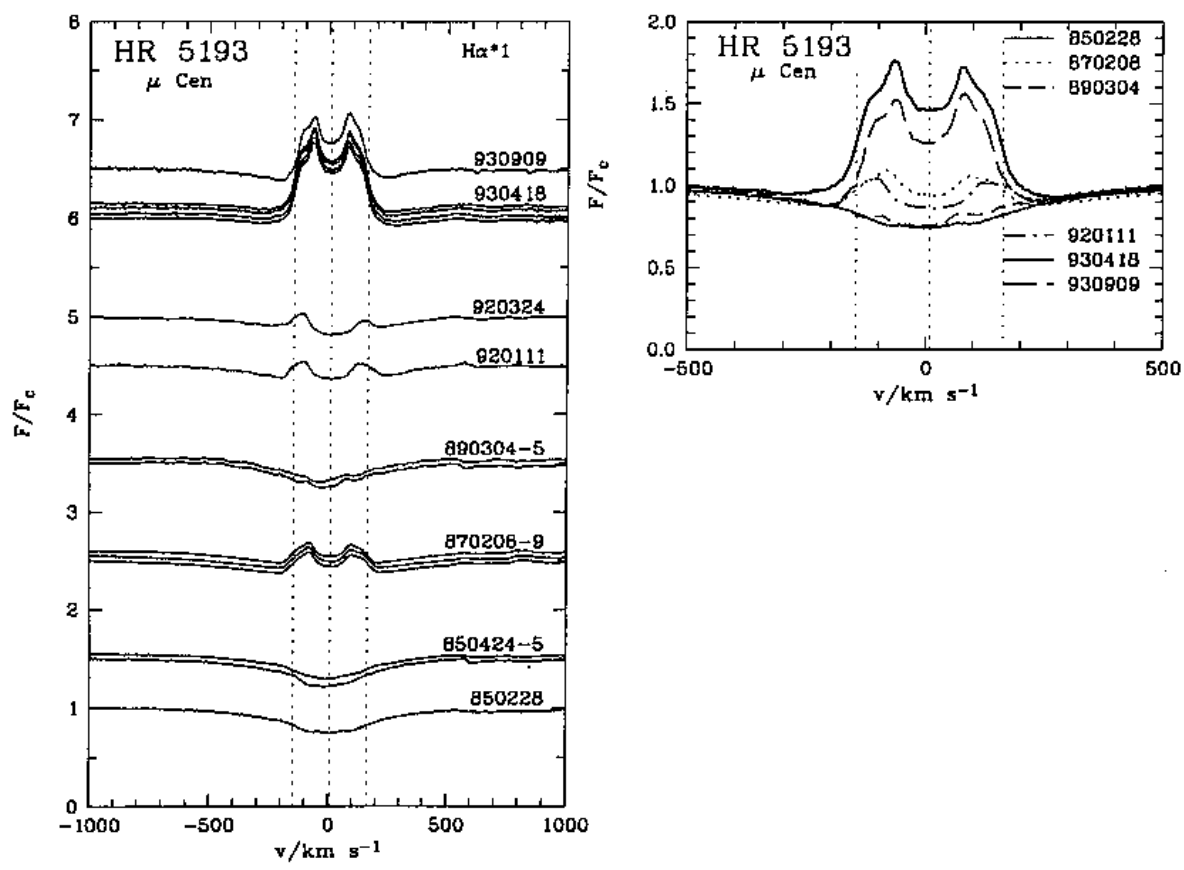

Fig. 55. H $\alpha$ profiles for HR 5193. This star is building up a new permanent disk since 1993
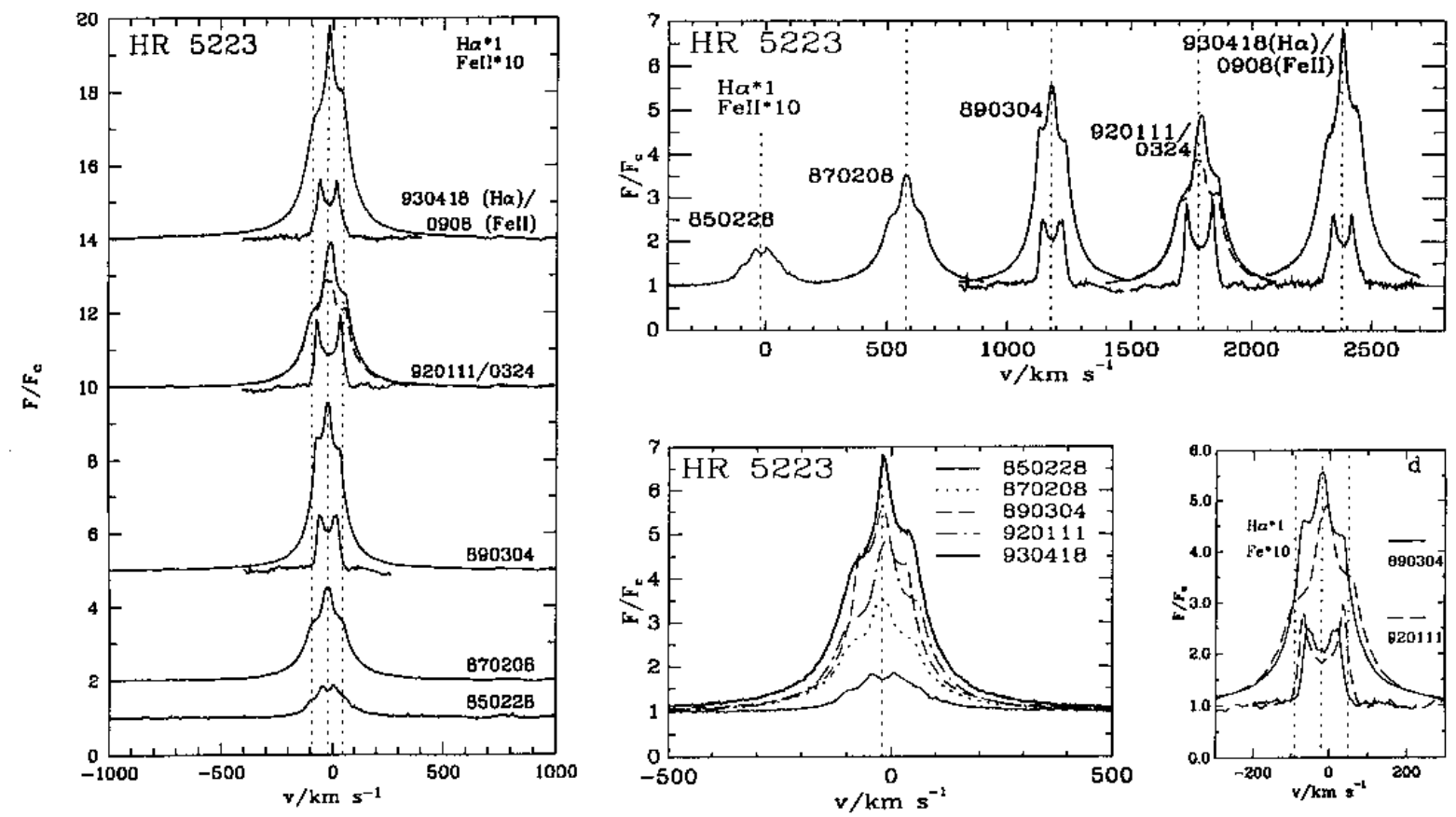

Fig. 56. $\mathrm{H} \alpha$ and Fe II profiles for $\mathrm{HR}$ 5223. Note the anticorrelation of $\mathrm{H} \alpha$ and Fe II strength and width in 1989 and 1992 

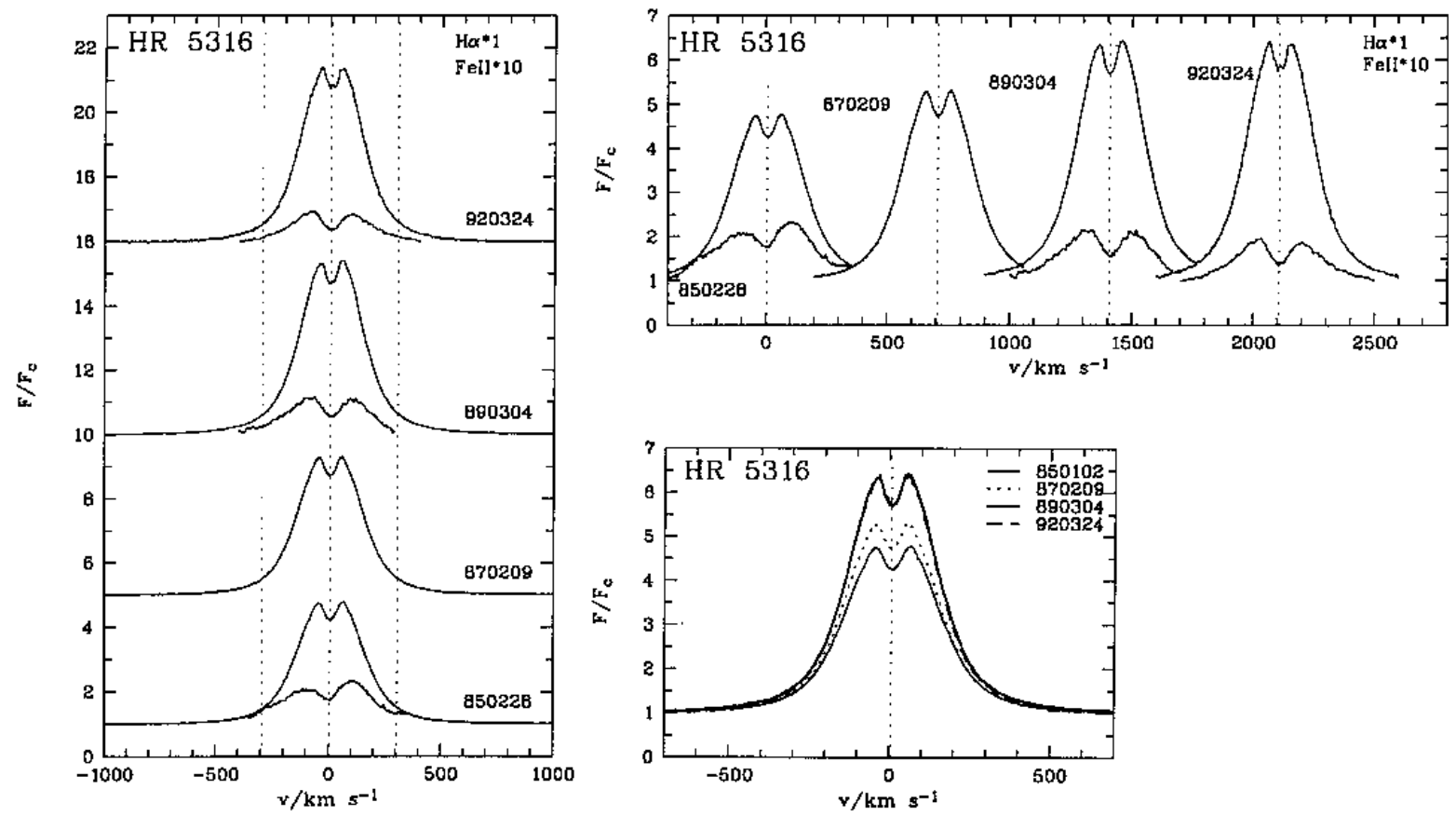

Fig. 57. $\mathrm{H} \alpha$ and $\mathrm{Fe}$ in profiles for $\mathrm{HR} 5316$
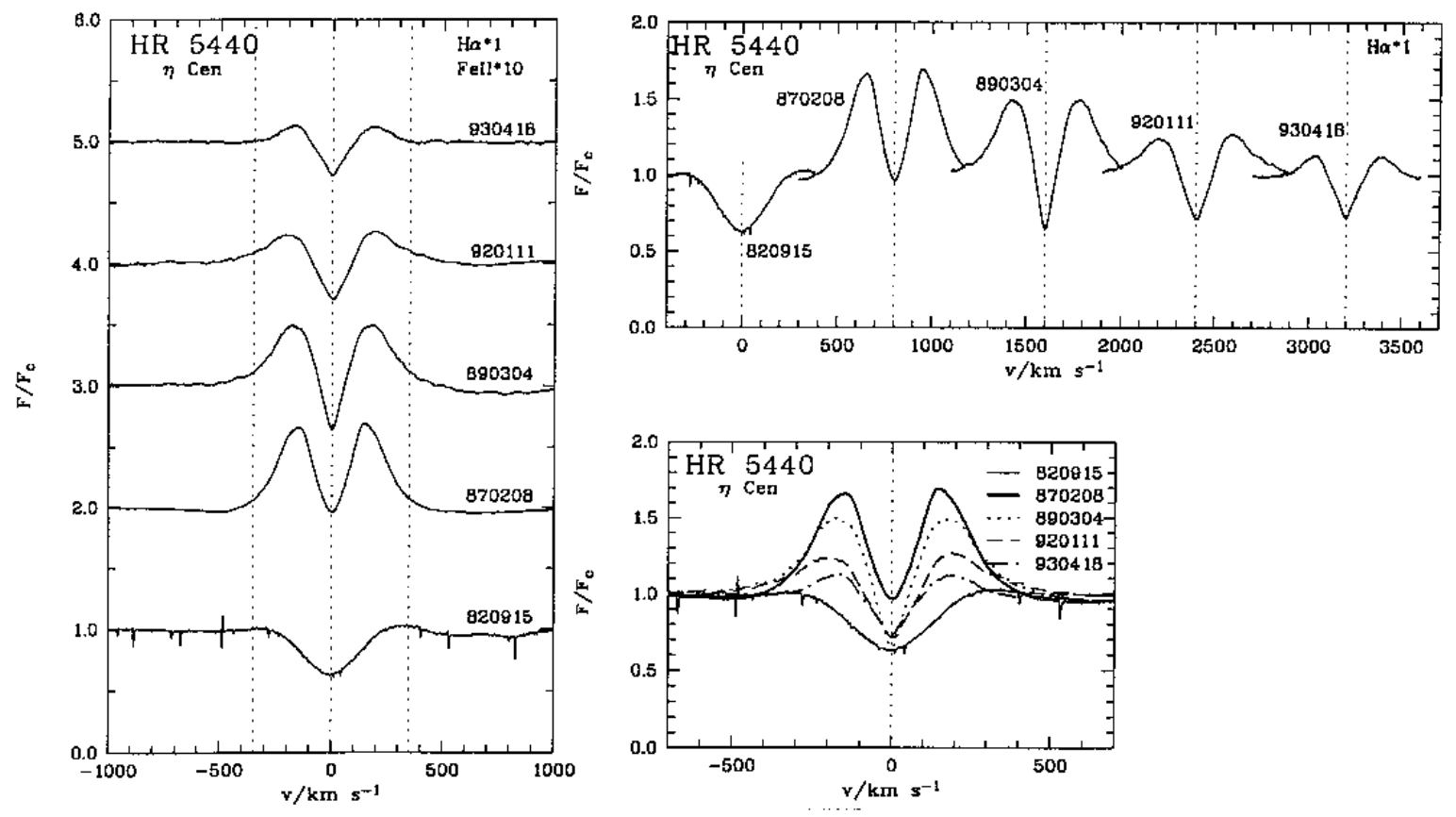

Fig. 58. $\mathrm{H} \alpha$ profiles for HR 5440 

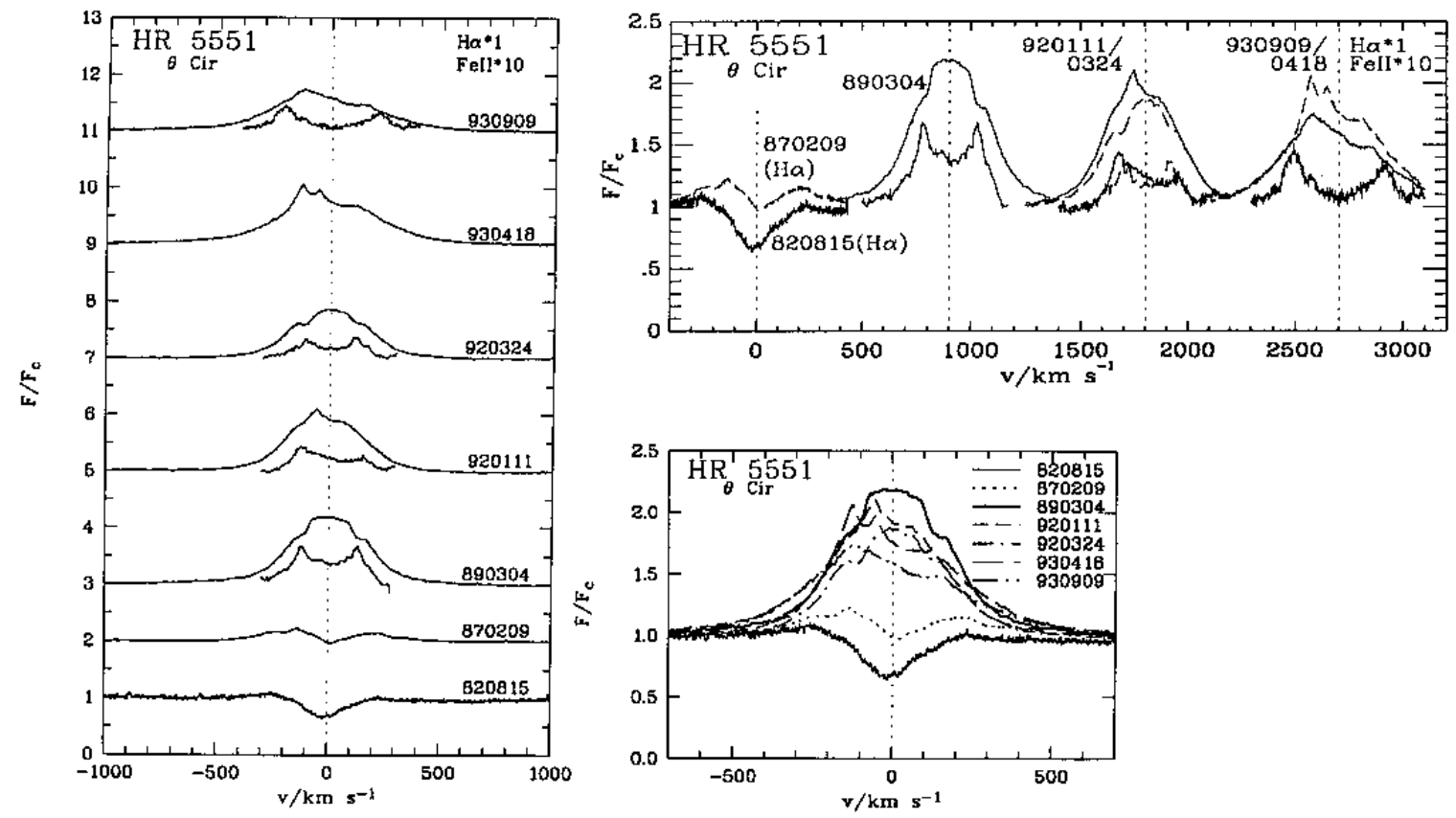

Fig. 59. H $\alpha$ and Fe II profiles for HR 5551. Vertical $v \sin i$ lines have been omitted here since Slettebak's (1982) value $\left(100 \mathrm{~km} \mathrm{~s}^{-1}\right)$ seems dramatically underestimated

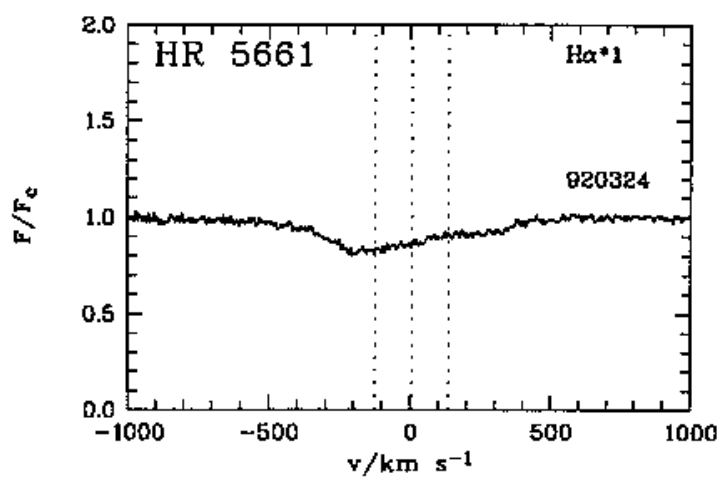

Fig. 60. $\mathrm{H} \alpha$ profile for HR 5661

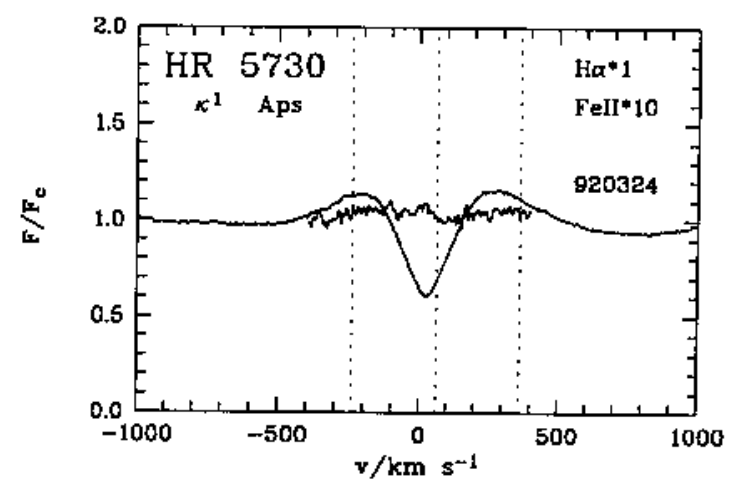

Fig. 61. $\mathrm{H} \alpha$ and $\mathrm{Fe}$ II profile for $\mathrm{HR} 5730$ 

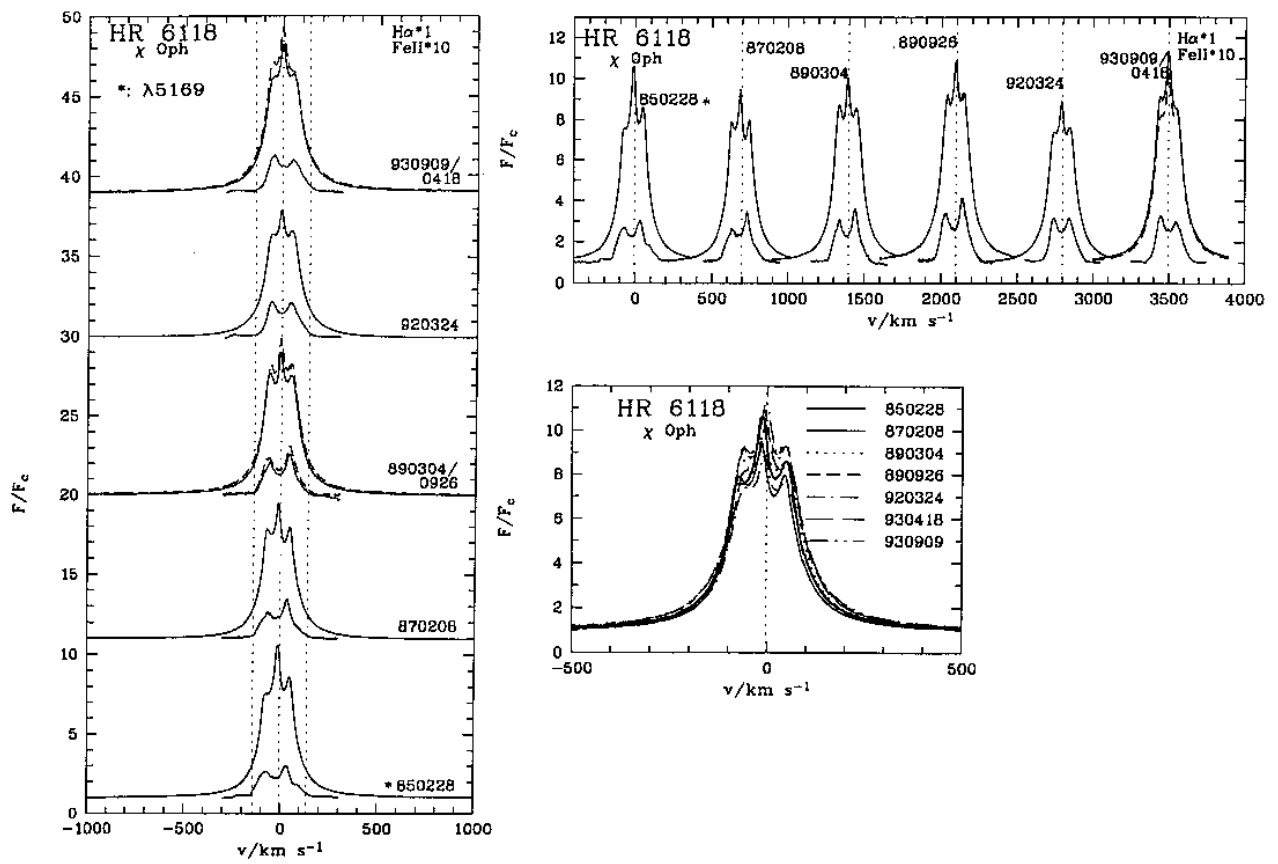

Fig. 62. $\mathrm{H} \alpha$ and Fe in profiles for $\mathrm{HR} 6118$
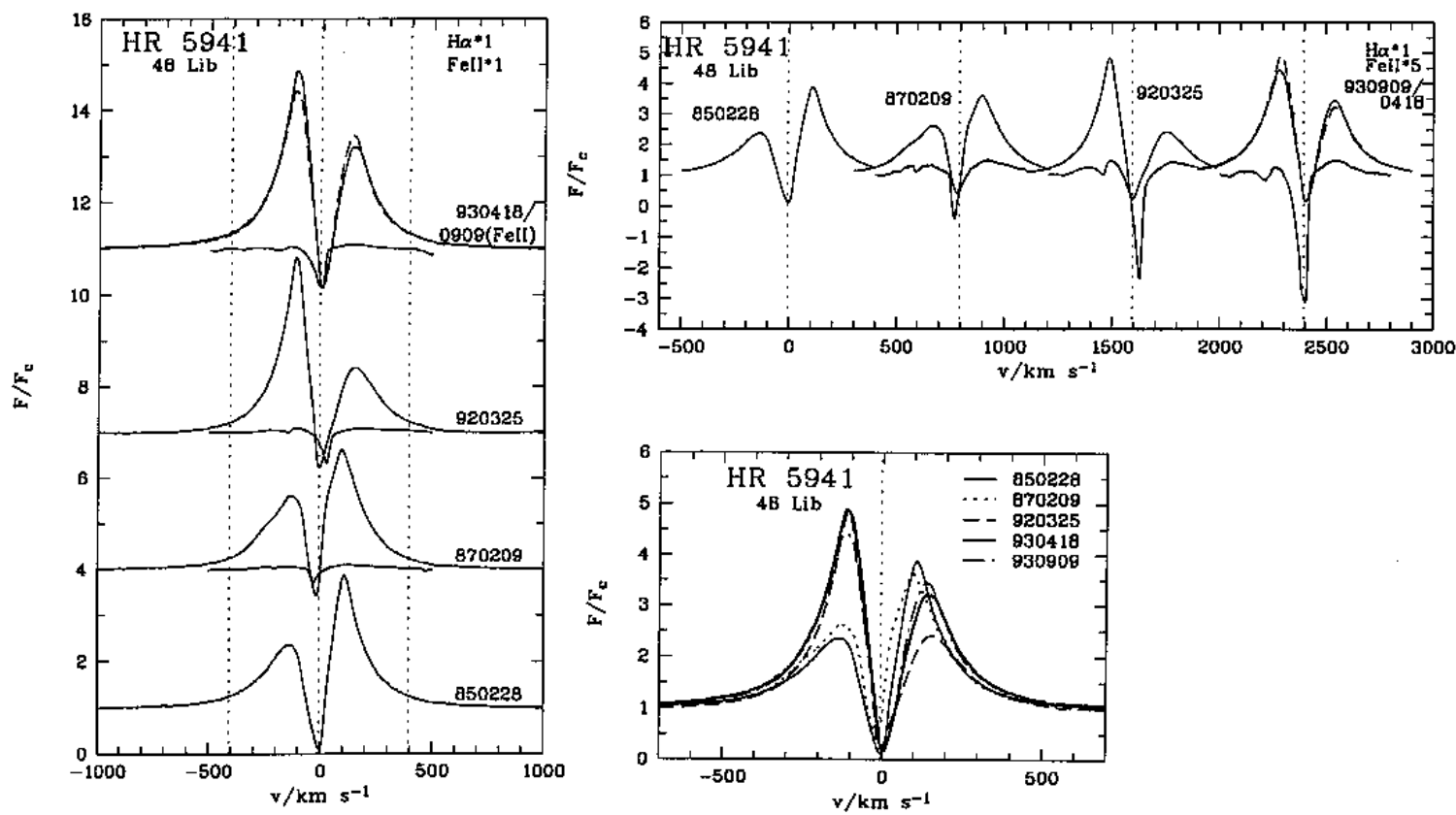

Fig. 63. $\mathrm{H} \alpha$ and Fe II profiles for HR 5941. Note that the scaling for Fe II lines is different in panels a and b, and that the depth of the Fe II troughs in panel $\mathrm{b}$ is affected by the expansion 

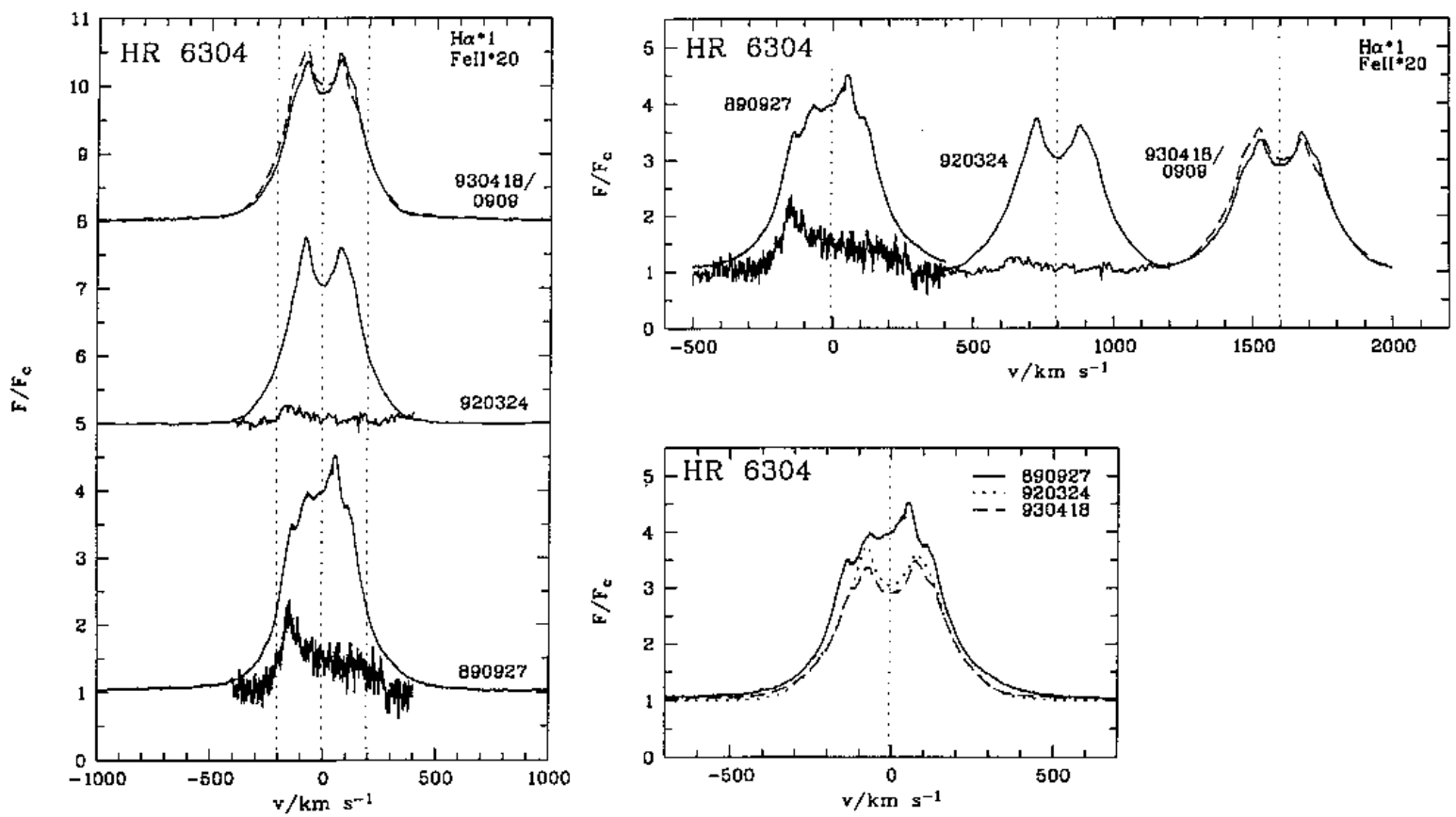

Fig. 64. $\mathrm{H} \alpha$ and $\mathrm{Fe}$ in profiles for $\mathrm{HR} 6304$
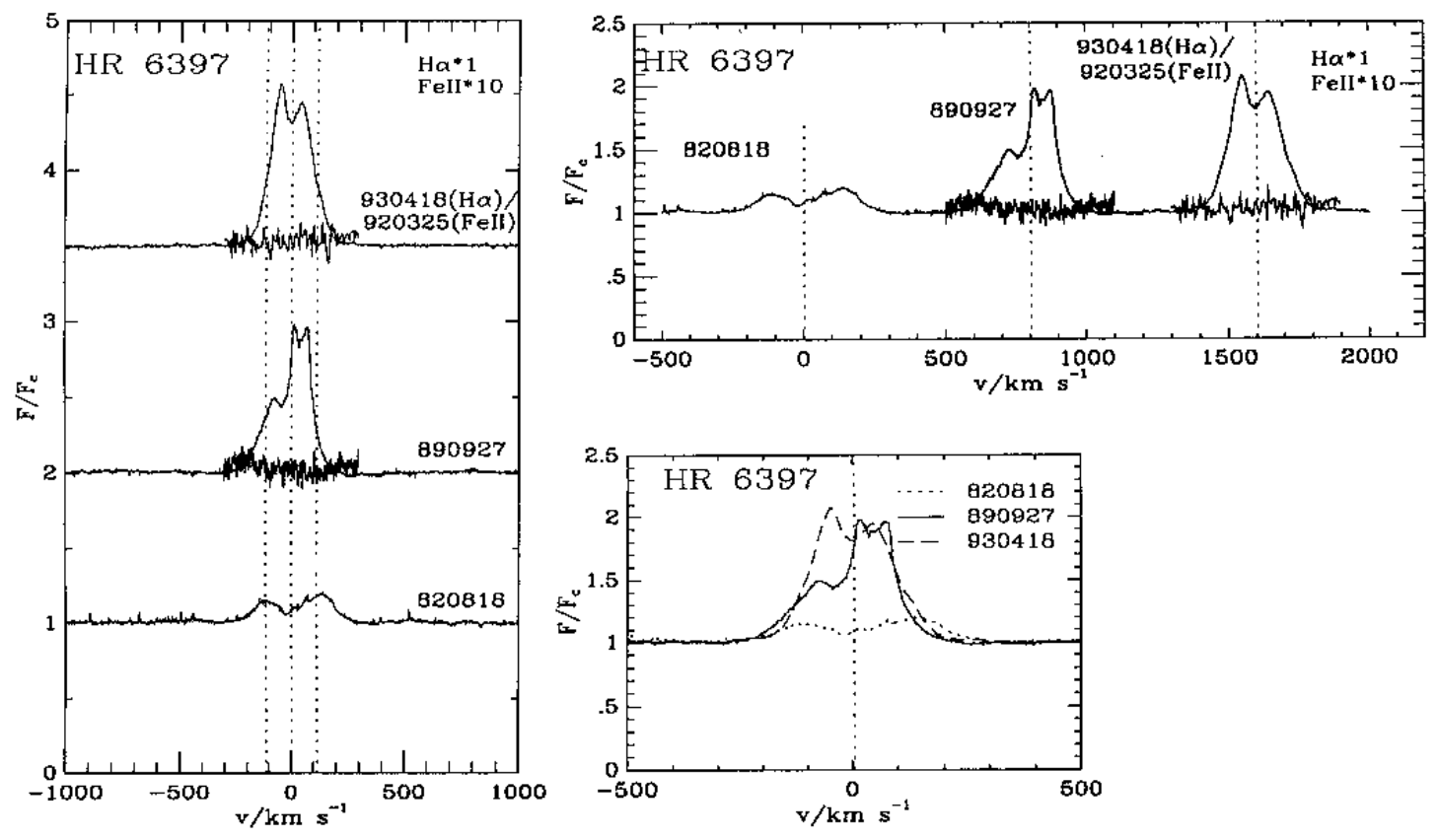

Fig. 65. $\mathrm{H} \alpha$ and $\lambda 5317$ profiles for HR 6397. No trace of Fe II was visible in 1989 and 1992 

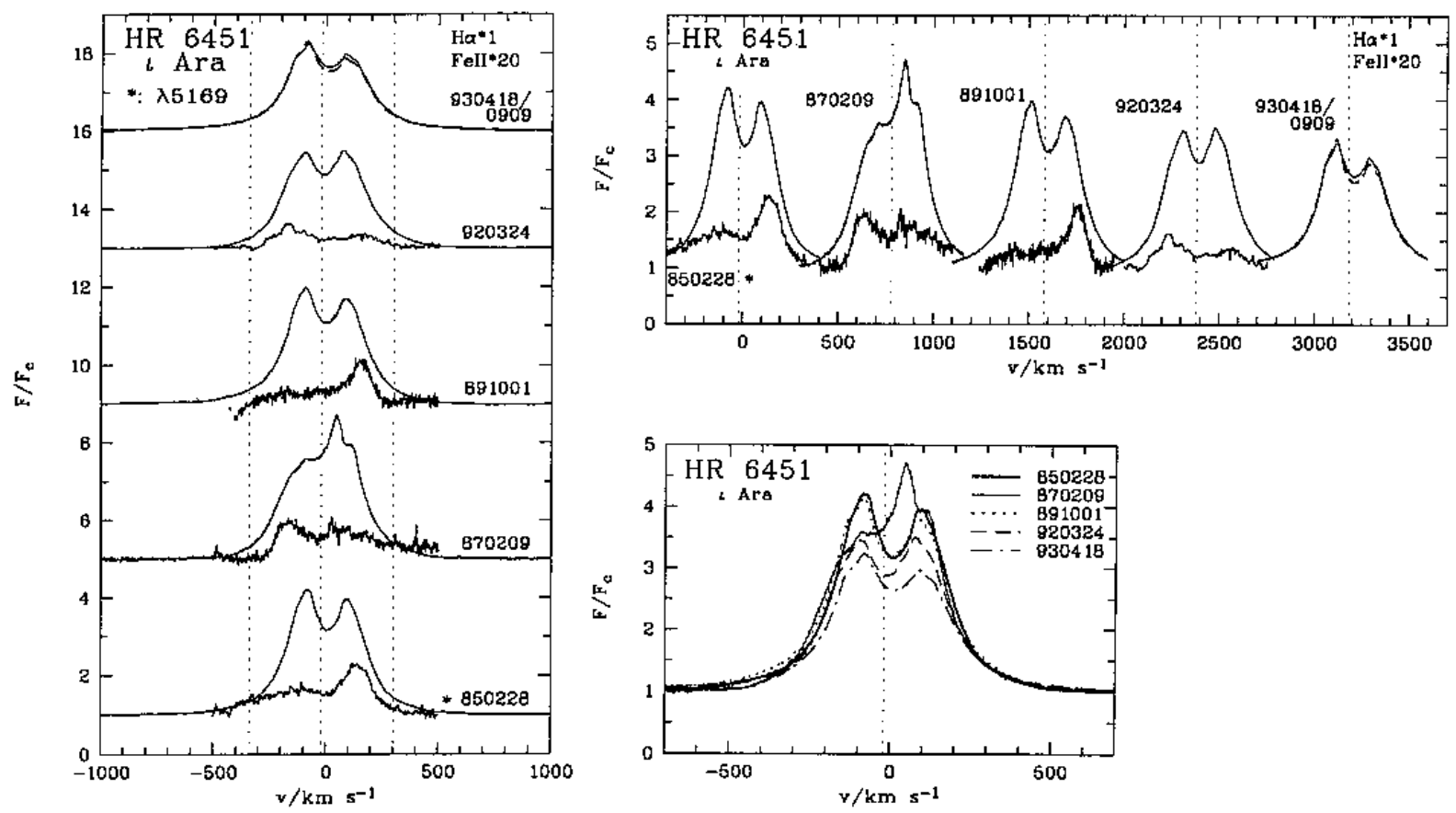

Fig. 66. $\mathrm{H} \alpha$ and $\mathrm{Fe}$ in profiles for $\mathrm{HR} 6451$
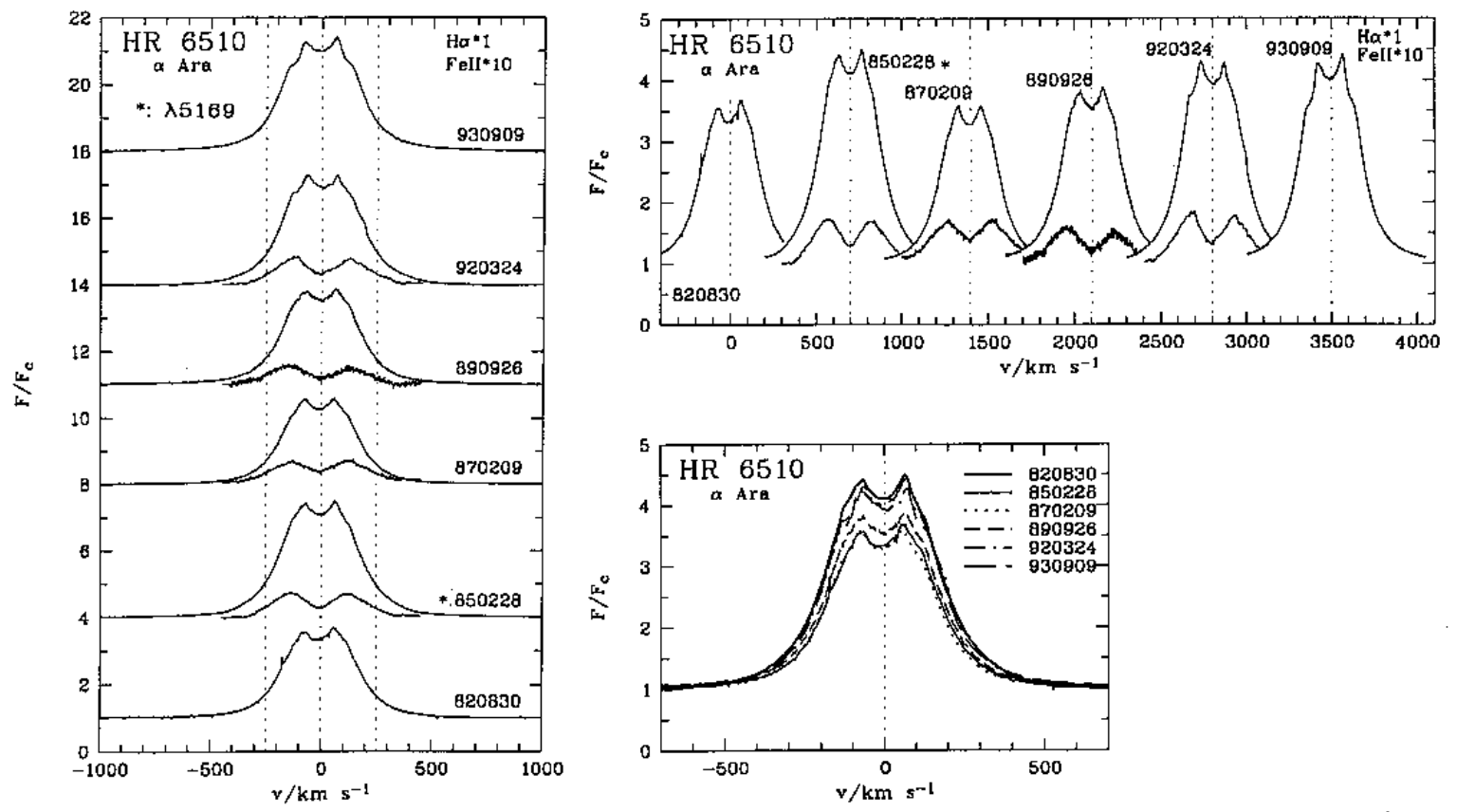

Fig. 67. $\mathrm{H} \alpha$ and $\mathrm{Fe}$ in profiles for $\mathrm{HR} 6510$ 

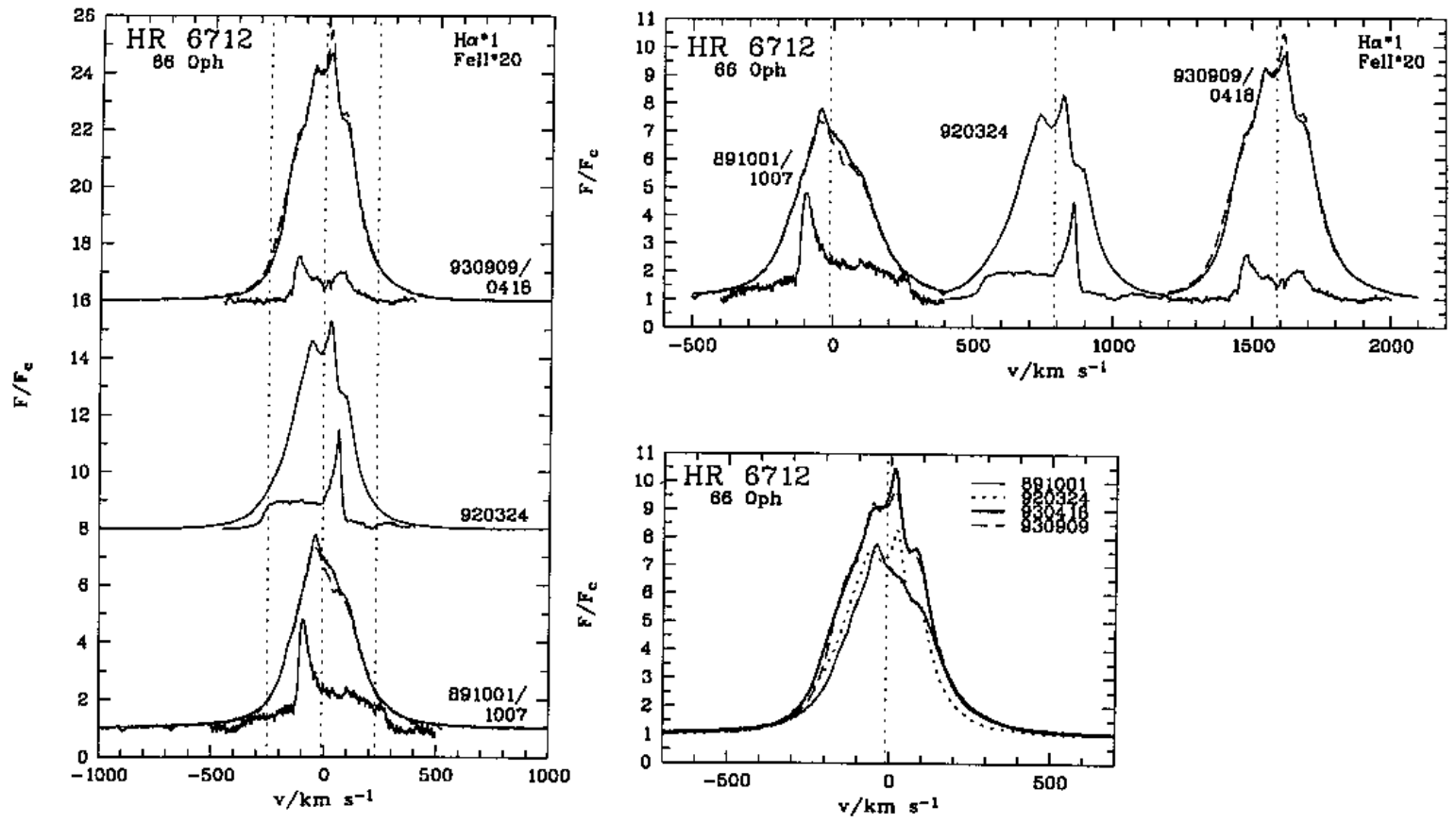

Fig. 68. $\mathrm{H} \alpha$ and $\mathrm{Fe}$ in profiles for $\mathrm{HR} 6712$

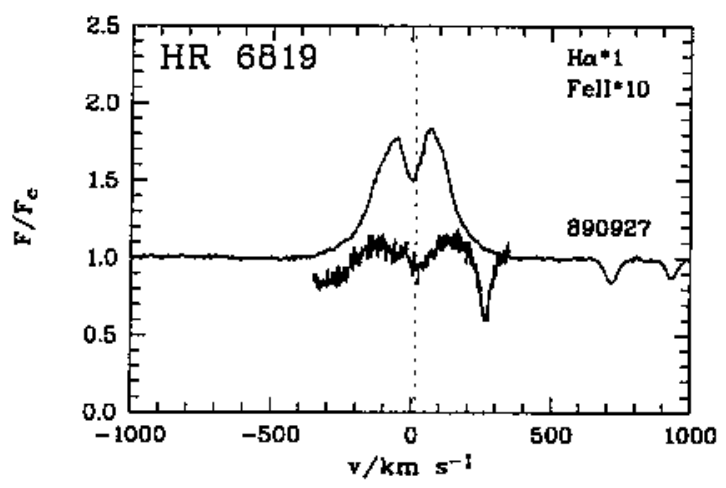

Fig. 69. $\mathrm{H} \alpha$ and Fe in profile for HR 6819 

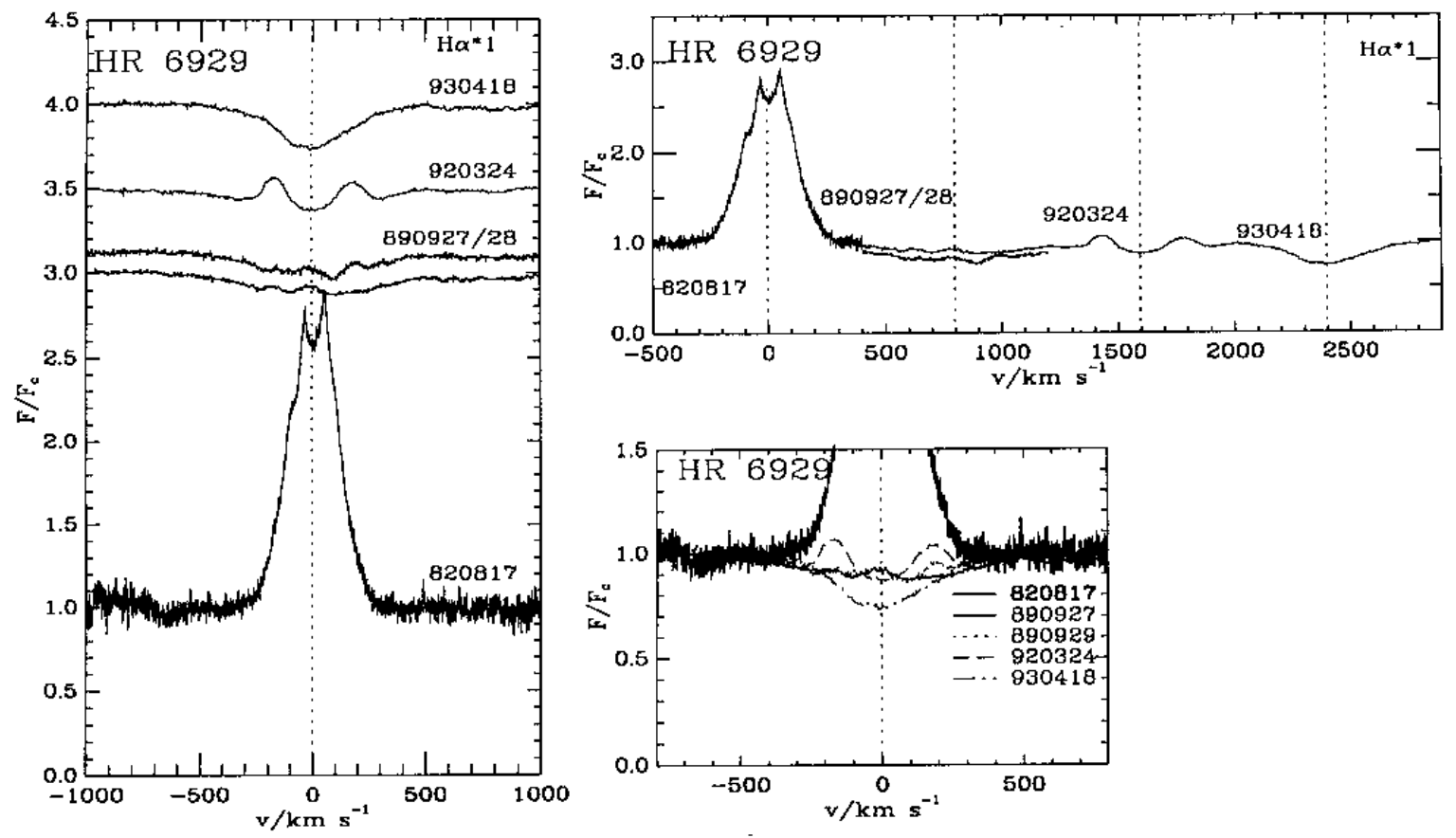

Fig. 70. H $\alpha$ profiles for HR 6929. Note that the $\mathrm{H} \alpha$ profile from 820817 has been cut off in panel c
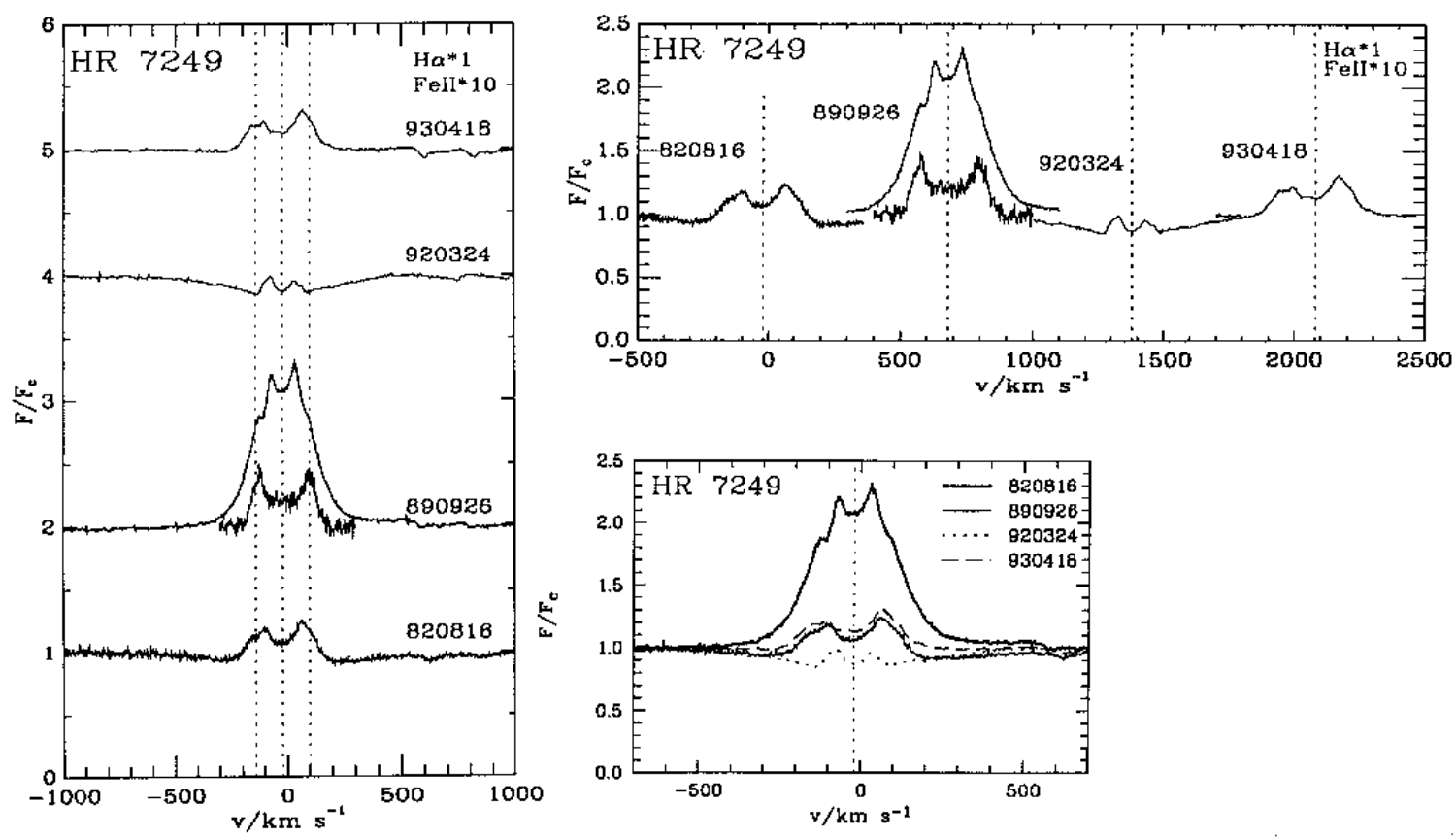

Fig. 71. $\mathrm{H} \alpha$ and $\mathrm{Fe}$ is profiles for $\mathrm{HR} 7249$ 


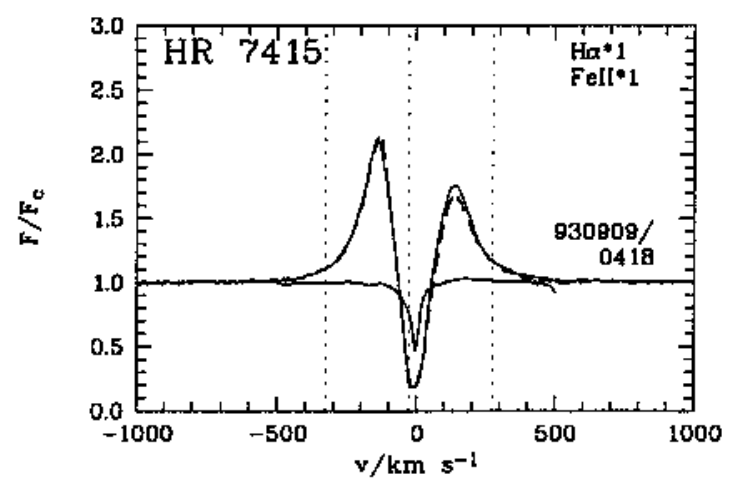

Fig. 72. H $\alpha$ and Fe is profiles for HR 7415
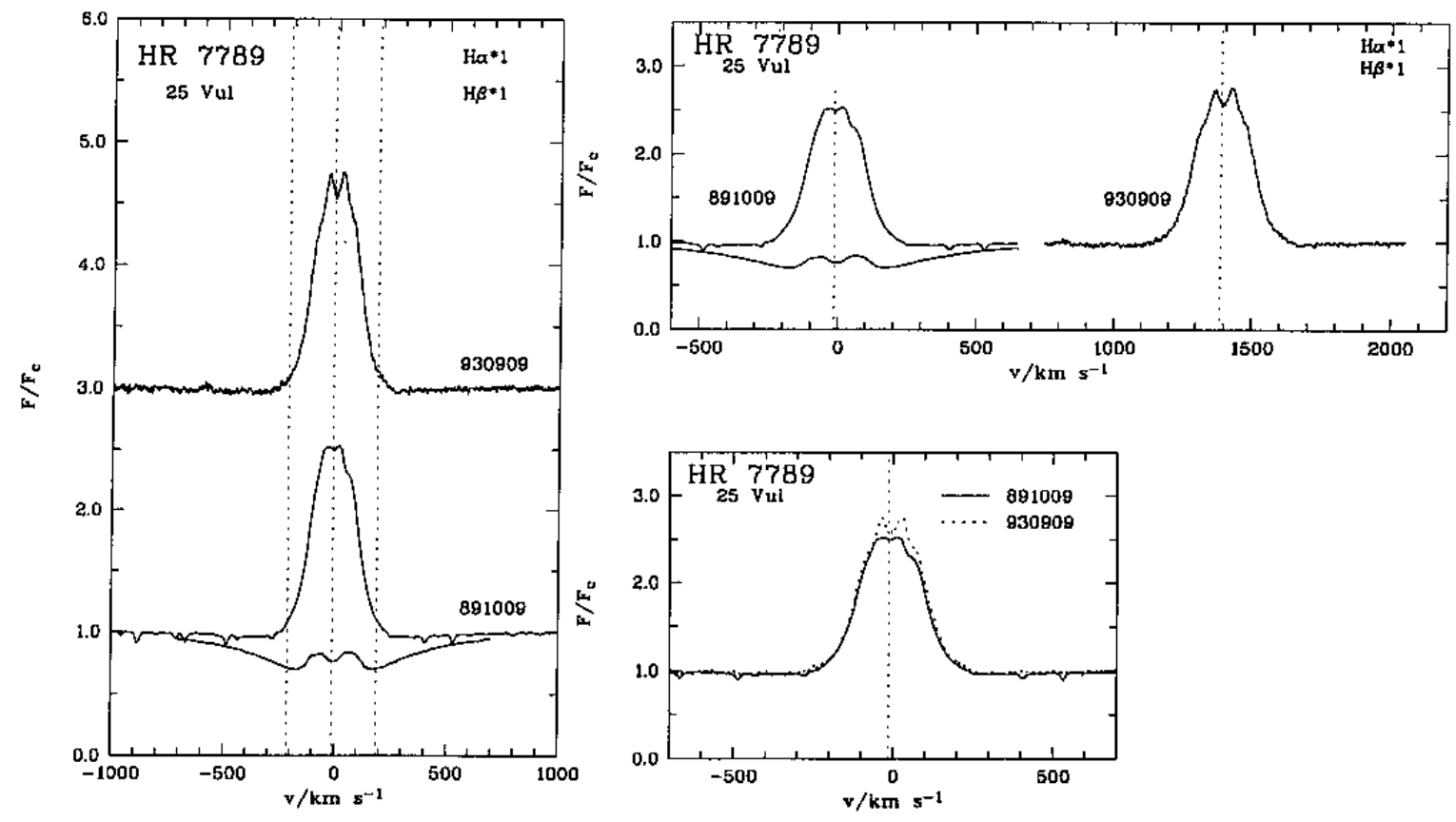

Fig. 73. $\mathrm{H} \alpha$ and $\mathrm{H} \beta$ profiles for $\mathrm{HR}$ 7789. Note that the 891009 profiles (from Sl92) are not fully resolved 

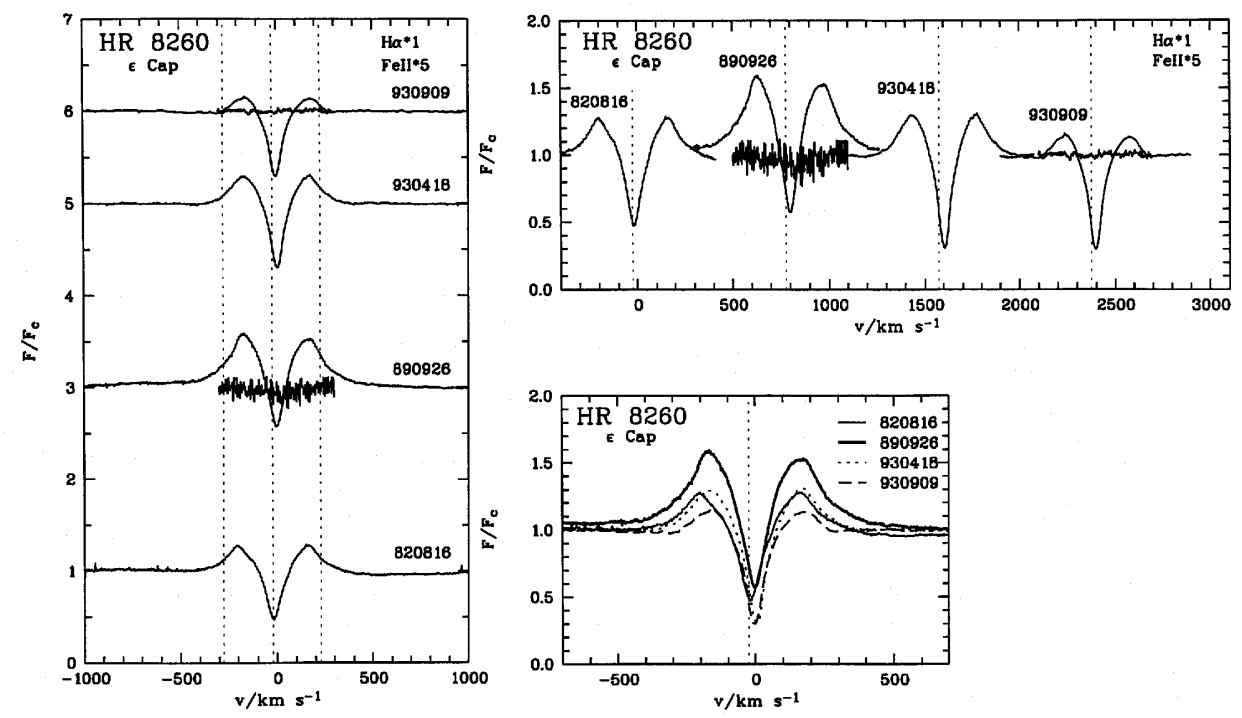

Fig. 74. H $\alpha$ and $\lambda 5317$ profiles for HR 8260. There is no trace of Fe II emission or shell absorption in the 1989 and 1993 data
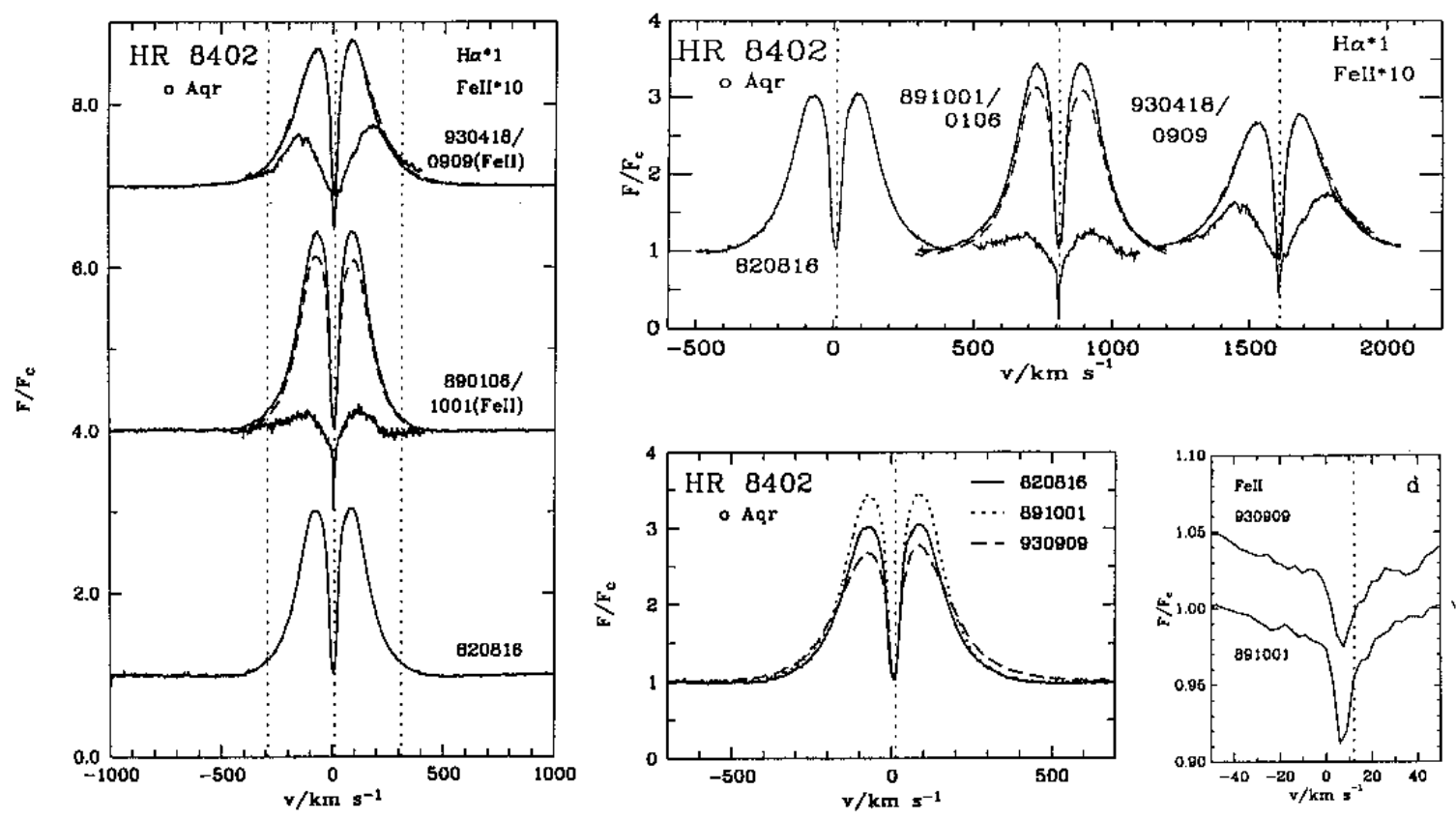

Fig. 75. $\mathrm{H} \alpha$ and Fe II profiles for HR 8402. Panel $\mathbf{d}$ shows a blow-up of the extremely narrow Fe II lines. Note that in panels a and $b$, the depth of the Fe II lines is affected by the expansion 

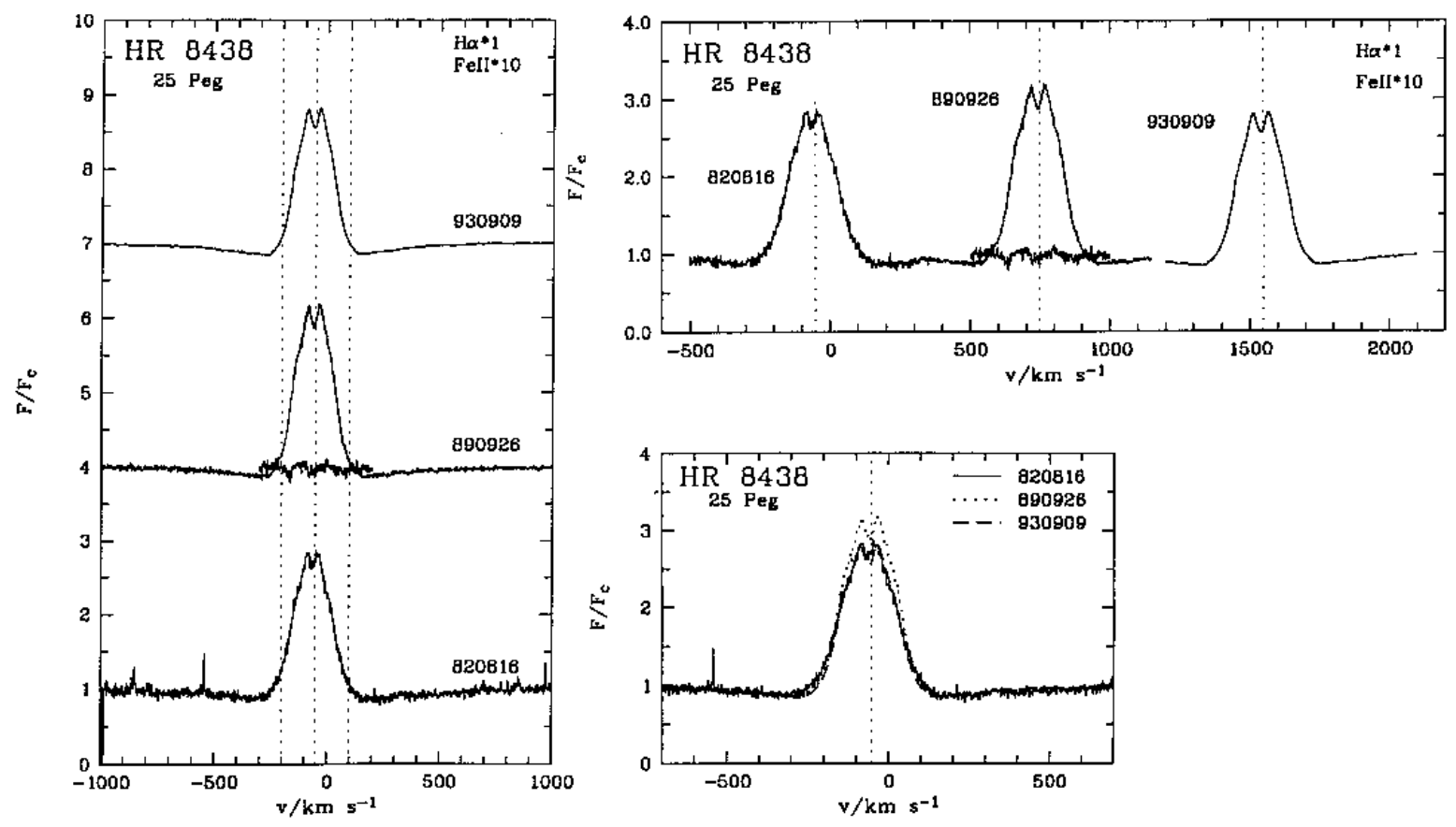

Fig. 76. $\mathrm{H} \alpha$ and $\lambda 5317$ profiles for HR 8438. No trace of Fe II emission is visible
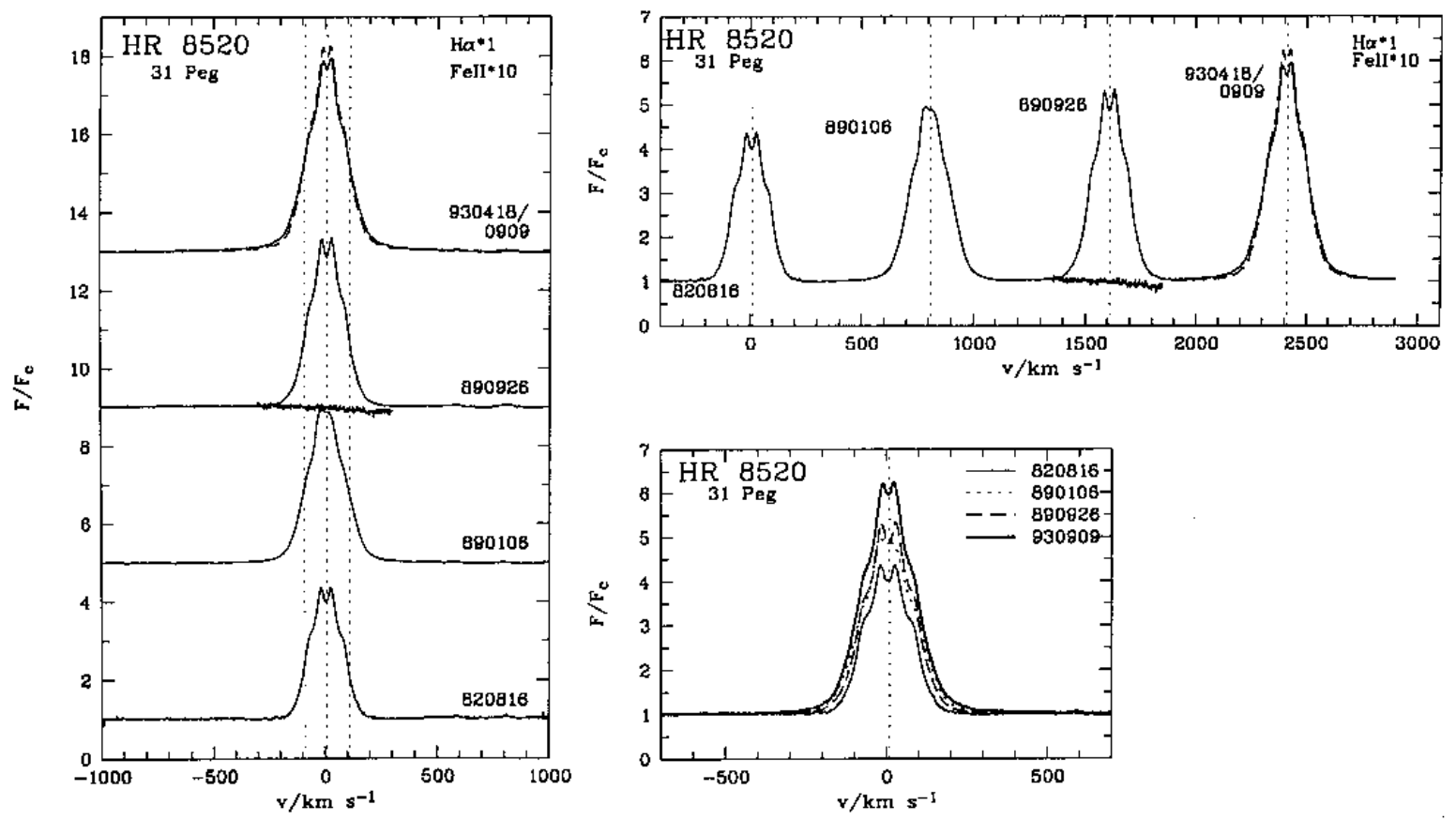

Fig. 77. $\mathrm{H} \alpha$ and $\lambda 5317$ profiles for HR 8520. No trace of Fe II emission is visible. The 890106 profile from Sl92 is not fully resolved 

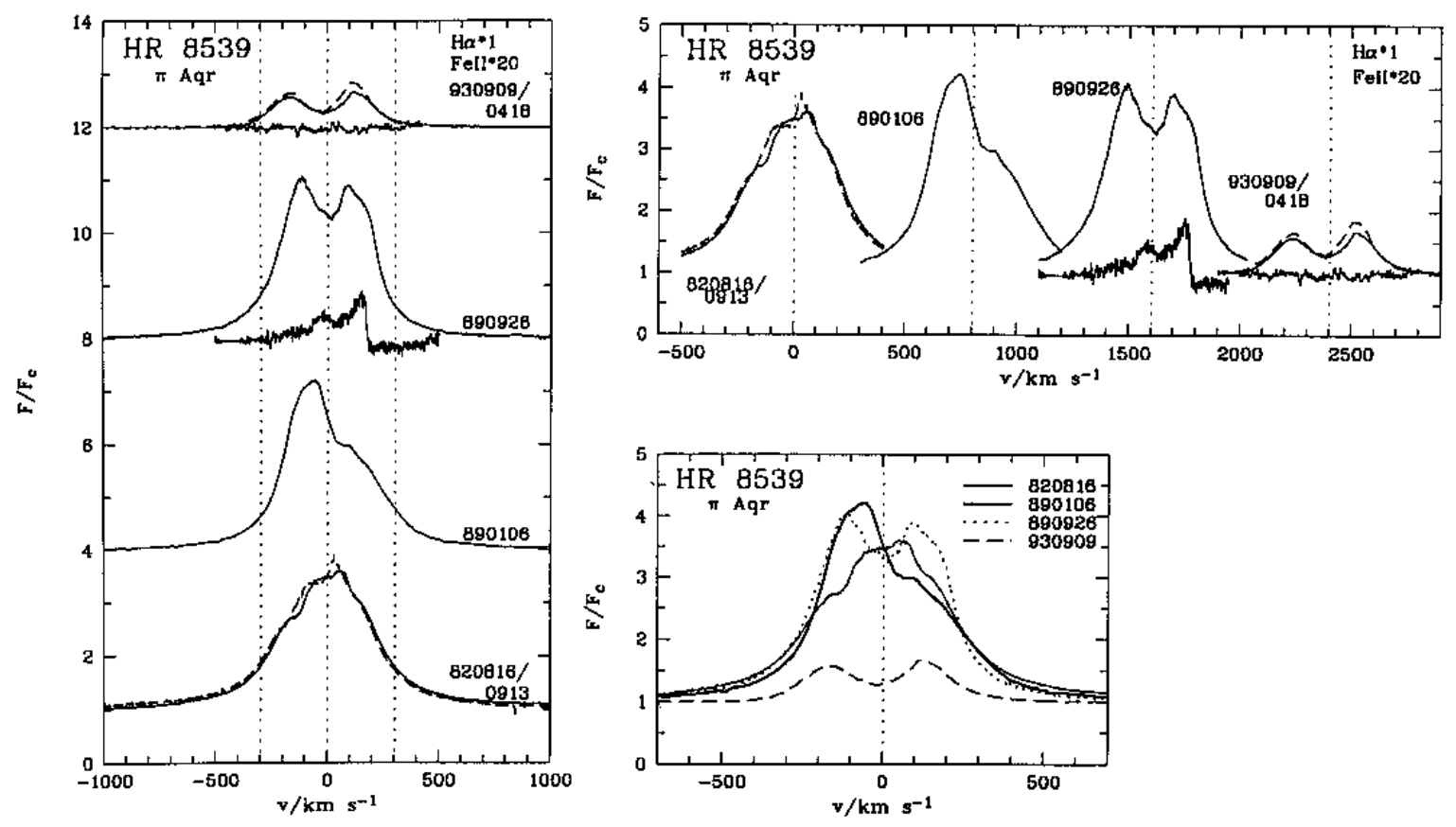

Fig. 78. $\mathrm{H} \alpha$ and Fe II profiles for HR 8539
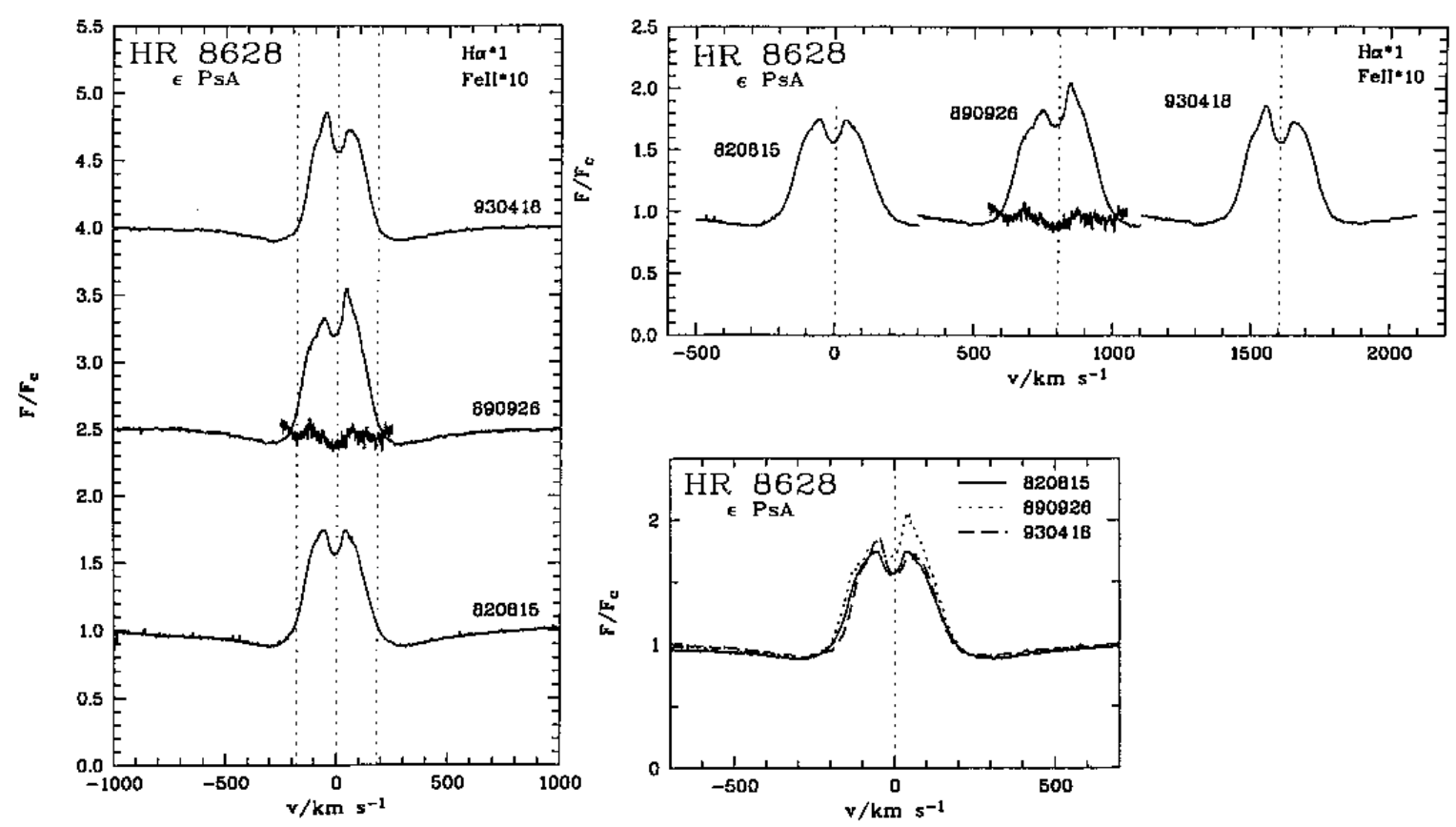

Fig. 79. $\mathrm{H} \alpha$ and $\mathrm{Fe}$ II profiles for $\mathrm{HR} 8628$. The Fe II emission is spurious 

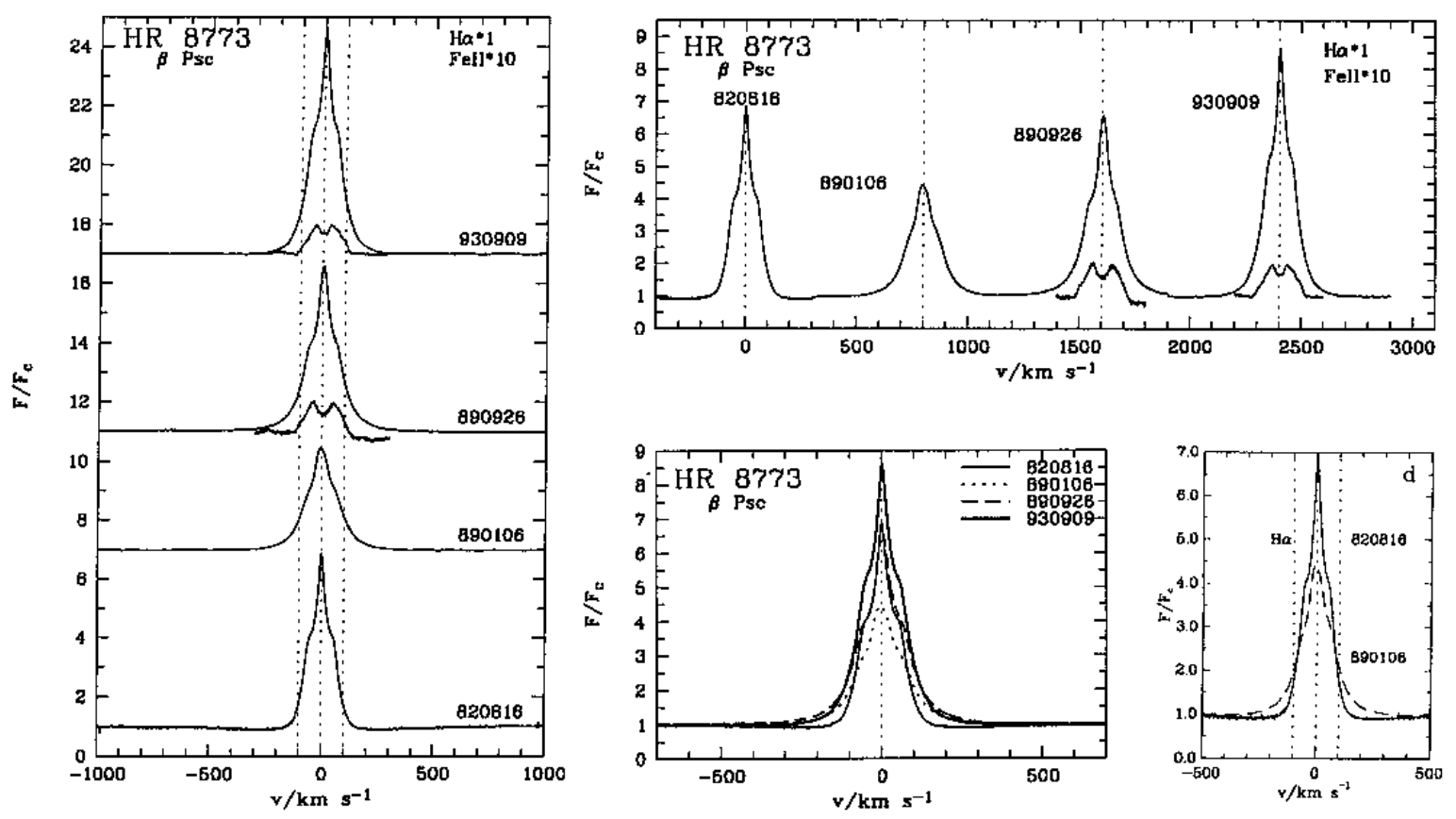

Fig. 80. $\mathrm{H} \alpha$ and Fe in profiles for HR 8773. Panel d shows the variations of $\mathrm{H} \alpha$ width and height between 1982 and 1989
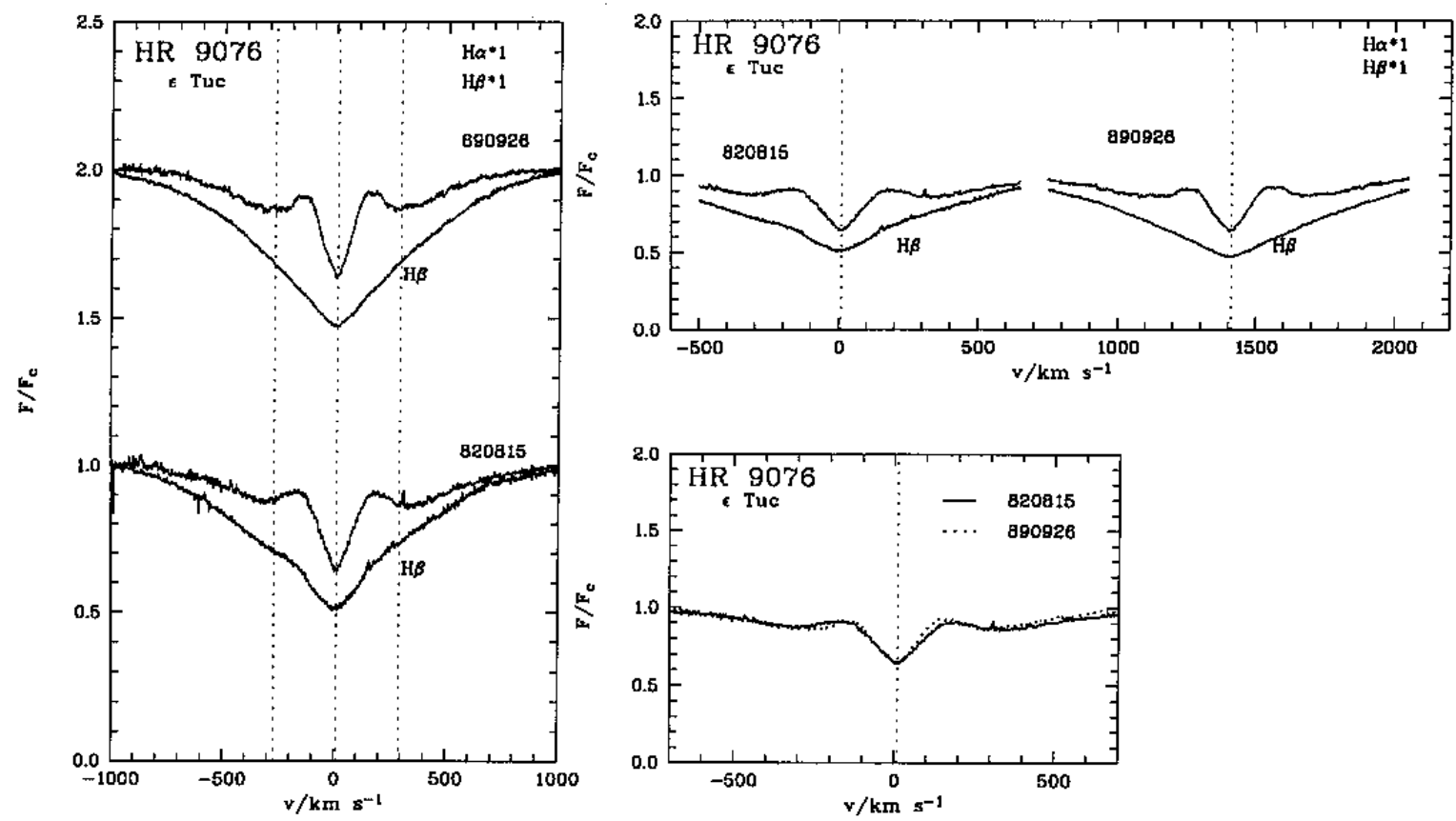

Fig. 81. $\mathrm{H} \alpha$ and $\mathrm{H} \beta$ profiles for HR 9076 


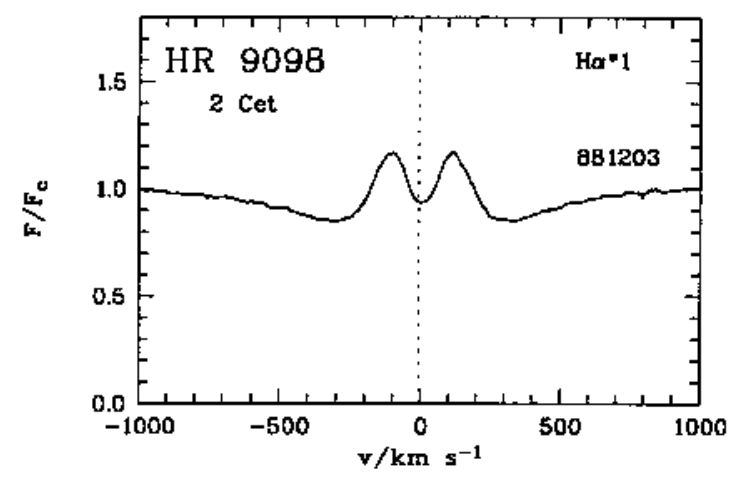

Fig. 82. $\mathrm{H} \alpha$ profile for HR 9098 